\title{
Disulfonimides Versus Phosphoric Acids in Brønsted Acid Catalysis: The Effect of Weak Hydrogen Bonds and Multiple Acceptors on Complex Structures and Reactivity
}

\author{
Kerstin Rothermel, ${ }^{\S}$ Matej Žabka, ${ }^{\S}$ Johnny Hioe and Ruth M. Gschwind ${ }^{*}$ \\ Institute of Organic Chemistry, \\ University of Regensburg, D-93053 Regensburg, Germany \\ e-mail: ruth.gschwind@chemie.uni-regensburg.de
}

Supplementary Material:

1 NMR Parameters and Spectra of the Binary DSI/imine Complexes........................................... 3

1.1 Experimental ${ }^{1} \mathrm{H}$ and ${ }^{15} \mathrm{~N}$ chemical shifts and ${ }^{1} \mathrm{~J}_{\mathrm{HN}}$ coupling constants ............................... 3

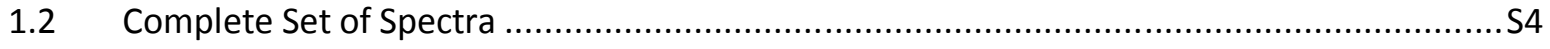

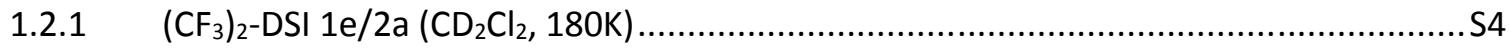

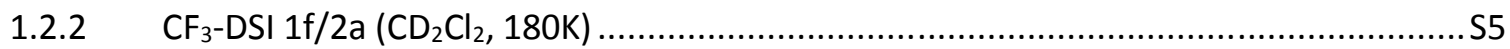

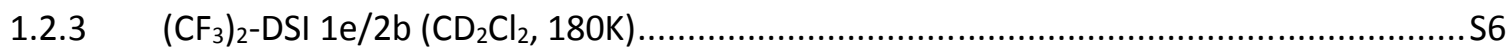

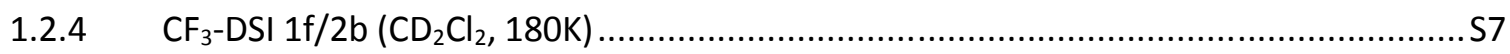

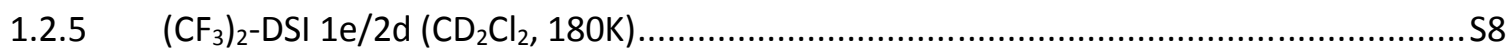

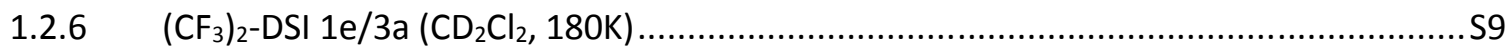

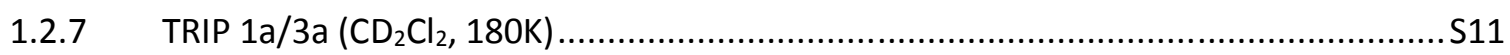

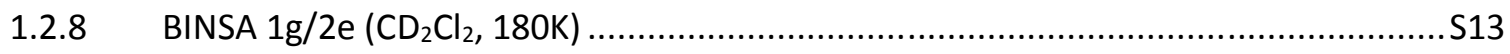

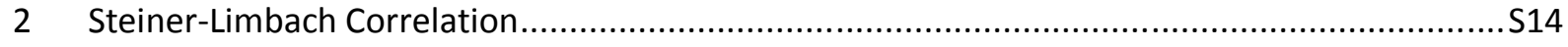

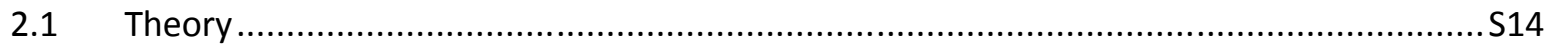

2.2 Used fit parameters for the Steiner-Limbach curve .................................................... 14

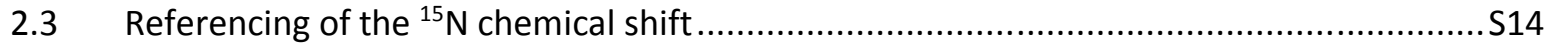

2.4 Steiner-Limbach curve with TRIP/3a-, $\left(\mathrm{CF}_{3}\right)_{2}$-DSI/3a- and BINSA/2e-complexes ................S15

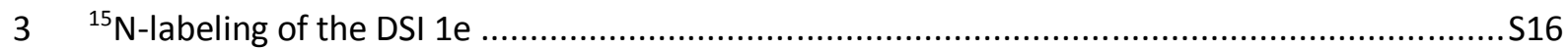

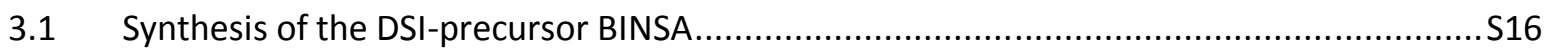

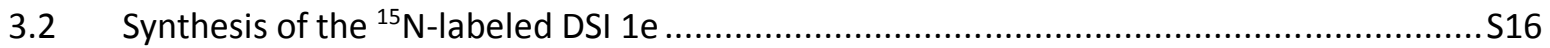

3.3 Comparison of the spectra of ${ }^{15} \mathrm{~N}$-labeld and not labeled $\left(\mathrm{CF}_{3}\right)_{2}-\mathrm{DSI} 1 \mathrm{e} / 2 \mathrm{a}\left(\mathrm{CD}_{2} \mathrm{Cl}_{2}, 180 \mathrm{~K}\right) . \mathrm{S} 17$

3.4 Comparison of the ${ }^{15} \mathrm{~N}$-spectra of the free $\left(\mathrm{CF}_{3}\right)_{2}$-DSI 1e and the $\left(\mathrm{CF}_{3}\right)_{2}$-DSI 1e/2a-binary

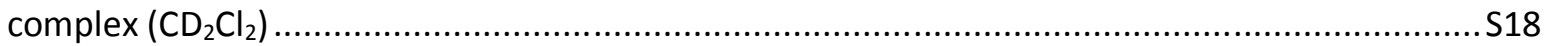


4 Calculated ${ }^{1} J_{\mathrm{HN}},{ }^{1 \mathrm{~h}} J_{\mathrm{NH}}$ and ${ }^{2 \mathrm{~h}} J_{\mathrm{NN}}$ Coupling Constants

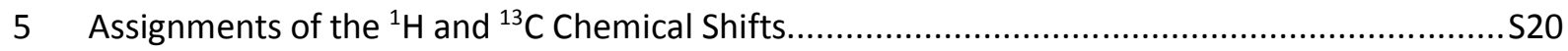

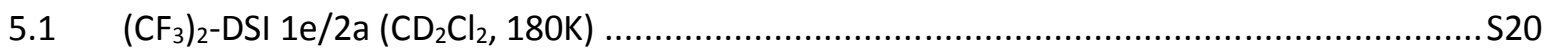

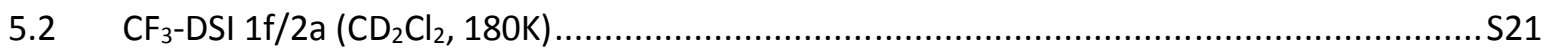

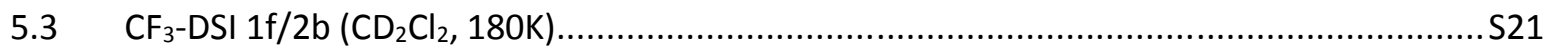

$6{ }^{1} \mathrm{H}$ Spectrum of a Binary DSI 1e-Complex with an Excess of Imine 2a (1:2) ............................S22

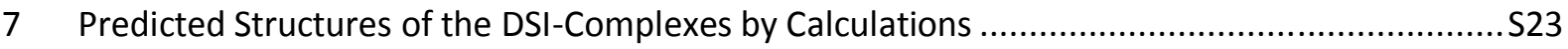

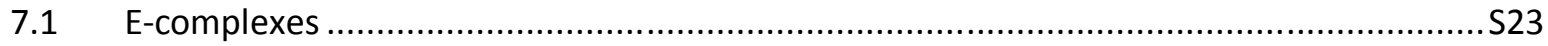

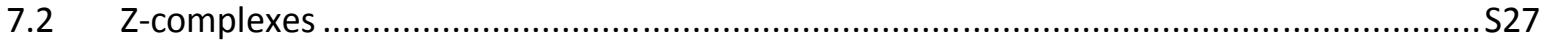

8 Structure Identification of All Binary Complexes .....................................................................

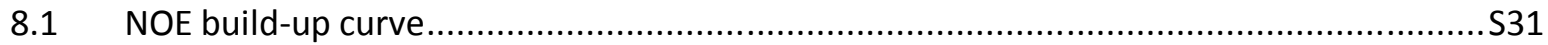

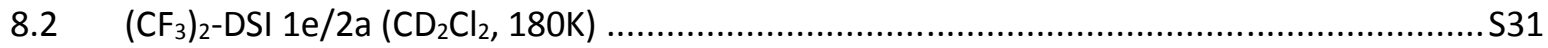

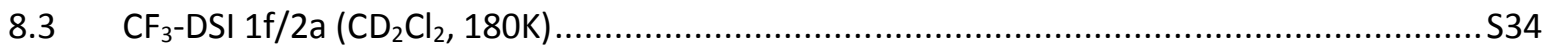

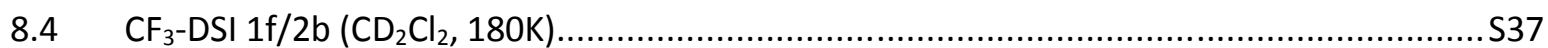

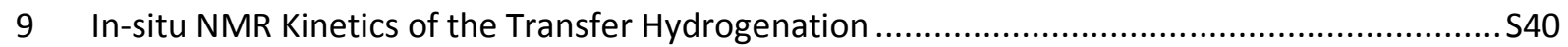

10 Ex-situ Reaction Kinetics of the Transfer Hydrogenation ....................................................

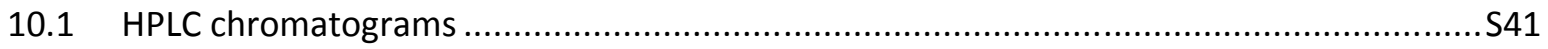

10.1.1 (E)-1-(4-methoxyphenyl)-N-phenylethan-1-imine 2a ..............................................

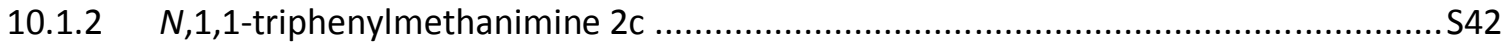

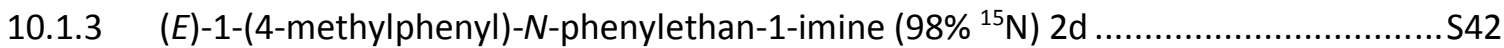

10.1.4 (E)-1-(4-trifluoromethylphenyl)- $N$-phenylethan-1-imine $\left(98 \%{ }^{15} \mathrm{~N}\right)$ 2e ...................S42

10.2 Enantiomeric Excess with Different Catalysts ...............................................................S43

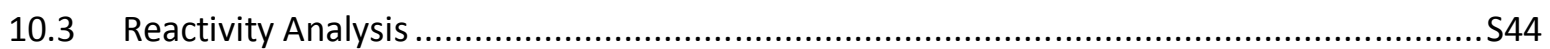

10.3.1 1-(4-methoxyphenyl)- $N$-phenylethan-1-amine 4a .....................................................

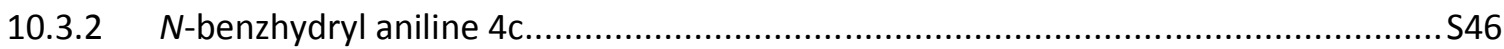

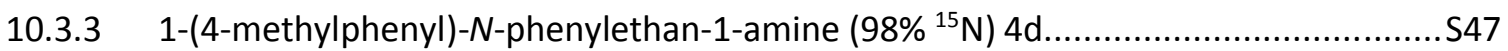

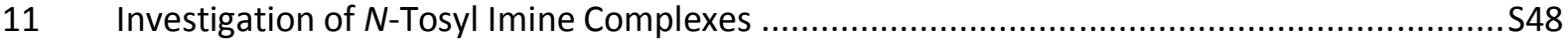

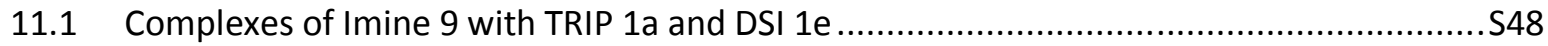

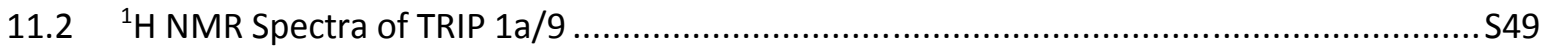

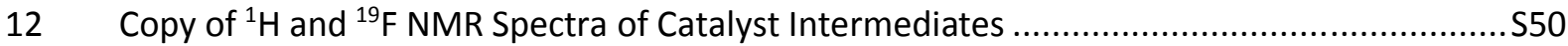

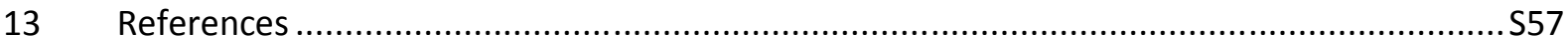




\section{NMR Parameters and Spectra of the Binary DSI/imine Complexes}

\subsection{Experimental ${ }^{1} \mathrm{H}$ and ${ }^{15} \mathrm{~N}$ chemical shifts and ${ }^{1} J_{H N}$ coupling constants}

Table S 1: NMR parameters $\left(\delta(\mathrm{NHN}), \delta(\mathrm{NHN})\right.$ and $\left.{ }^{1} J_{\mathrm{HN}}\right)$ of $\mathrm{DSl} /$ imine-complexes in $\mathrm{CD}_{2} \mathrm{Cl}_{2}$ at $180 \mathrm{~K}$. [a] value obtained from ${ }^{1} \mathrm{H},{ }^{15} \mathrm{~N}-\mathrm{HMBC}$; [b] at $230 \mathrm{~K}$; [c] at $170 \mathrm{~K}$; [d] broad, overlapping peak. (n.d. = not determined)

\begin{tabular}{|c|c|c|c|c|}
\hline & & $\begin{array}{c}\delta(\mathrm{NH} N) \\
{[\mathrm{ppm}]}\end{array}$ & $\begin{array}{c}\delta(\mathrm{NHN}) \\
{[\mathrm{ppm})}\end{array}$ & $\begin{array}{c}{ }^{1} J_{\mathrm{HN}}{ }^{[\mathrm{b}]} \\
{[\mathrm{Hz}]}\end{array}$ \\
\hline$\left(\mathrm{CF}_{3}\right)_{2}-\mathrm{DSI} \mathbf{1 e / 2 a}$ & $E$ & 12.86 & 188.70 & $90.6^{[\mathrm{b}]}$ \\
\hline$\left(\mathrm{CF}_{3}\right)_{2}-\mathrm{DSI} \mathbf{1 e / 2 a}$ & $Z$ & 13.92 & 196.4 & $88.8^{[\mathrm{b}]}$ \\
\hline $\mathrm{CF}_{3}-\mathrm{DSI} \mathbf{1 f} / \mathbf{2 a}$ & $E$ & 13.06 & 190.20 & n.d. \\
\hline $\mathrm{CF}_{3}-\mathrm{DSI} \mathbf{1} \mathbf{f} / \mathbf{2 a}$ & $Z$ & 14.18 & 197.4 & $88.8^{[\mathrm{c}]}$ \\
\hline$\left(\mathrm{CF}_{3}\right)_{2}-\mathrm{DSI} \mathbf{1 e / 2 b}$ & $E$ & 14.24 & n.d. & - \\
\hline$\left(\mathrm{CF}_{3}\right)_{2}-\mathrm{DSI} \mathbf{1} \mathbf{e} / \mathbf{2} \mathbf{b}$ & $Z$ & 14.99 & $205.8^{[a]}$ & $\sim 80.6^{[a]}$ \\
\hline $\mathrm{CF}_{3}$-DSI $\mathbf{1 f} / \mathbf{2 b}$ & $E$ & 14.66 & n.d. & - \\
\hline $\mathrm{CF}_{3}$-DSI $\mathbf{1 f} / \mathbf{2 b}$ & $Z$ & 15.16 & n.d. & - \\
\hline$\left(\mathrm{CF}_{3}\right)_{2}-\mathrm{DSI} 1 \mathrm{e} / 2 \mathrm{~d}$ & $E$ & 13.84 & 197.7 & 88.7 \\
\hline$\left(\mathrm{CF}_{3}\right)_{2}$-DSI $1 \mathrm{e} / 2 \mathrm{~d}$ & $Z$ & 14.26 & 201.6 & 87.5 \\
\hline$\left(\mathrm{CF}_{3}\right)_{2}-\mathrm{DSI} 1 \mathrm{e} / 2 \mathrm{e}$ & $E$ & $14.42^{[\mathrm{d}]}$ & $211.1^{[\mathrm{d}]}$ & n.d. \\
\hline$\left(\mathrm{CF}_{3}\right)_{2}-\mathrm{DSI} \mathbf{1 e / 2 e}$ & $Z$ & $15.04^{[\mathrm{d}]}$ & n.d. & n.d. \\
\hline$\left(\mathrm{CF}_{3}\right)_{2}-\mathrm{DSI} 1 \mathrm{e} / 3 \mathrm{a}$ & $E$ & 11.81 & $182.8^{[\mathrm{a}]}$ & $91-92^{[a]}$ \\
\hline$\left(\mathrm{CF}_{3}\right)_{2}-\mathrm{DSI} \mathbf{1 e / 3 a}$ & $Z$ & 12.56 & $190.5^{[\mathrm{a}]}$ & - \\
\hline TRIP 1a/3a & $E$ & 14.74 & $195.4^{[\mathrm{a}]}$ & $84.6^{[a]}$ \\
\hline TRIP 1a/3a & $Z$ & 15.36 & $201.5^{[a]}$ & - \\
\hline BINSA $1 \mathrm{~g} / \mathbf{2 e}$ & $E$ & 13.13 & 202.1 & 90.2 \\
\hline
\end{tabular}




\subsection{Complete Set of Spectra}

\subsection{1 $\left(\mathrm{CF}_{3}\right)_{2}$-DSI 1e/2a $\left(\mathrm{CD}_{2} \mathrm{Cl}_{2}, 180 \mathrm{~K}\right)$}
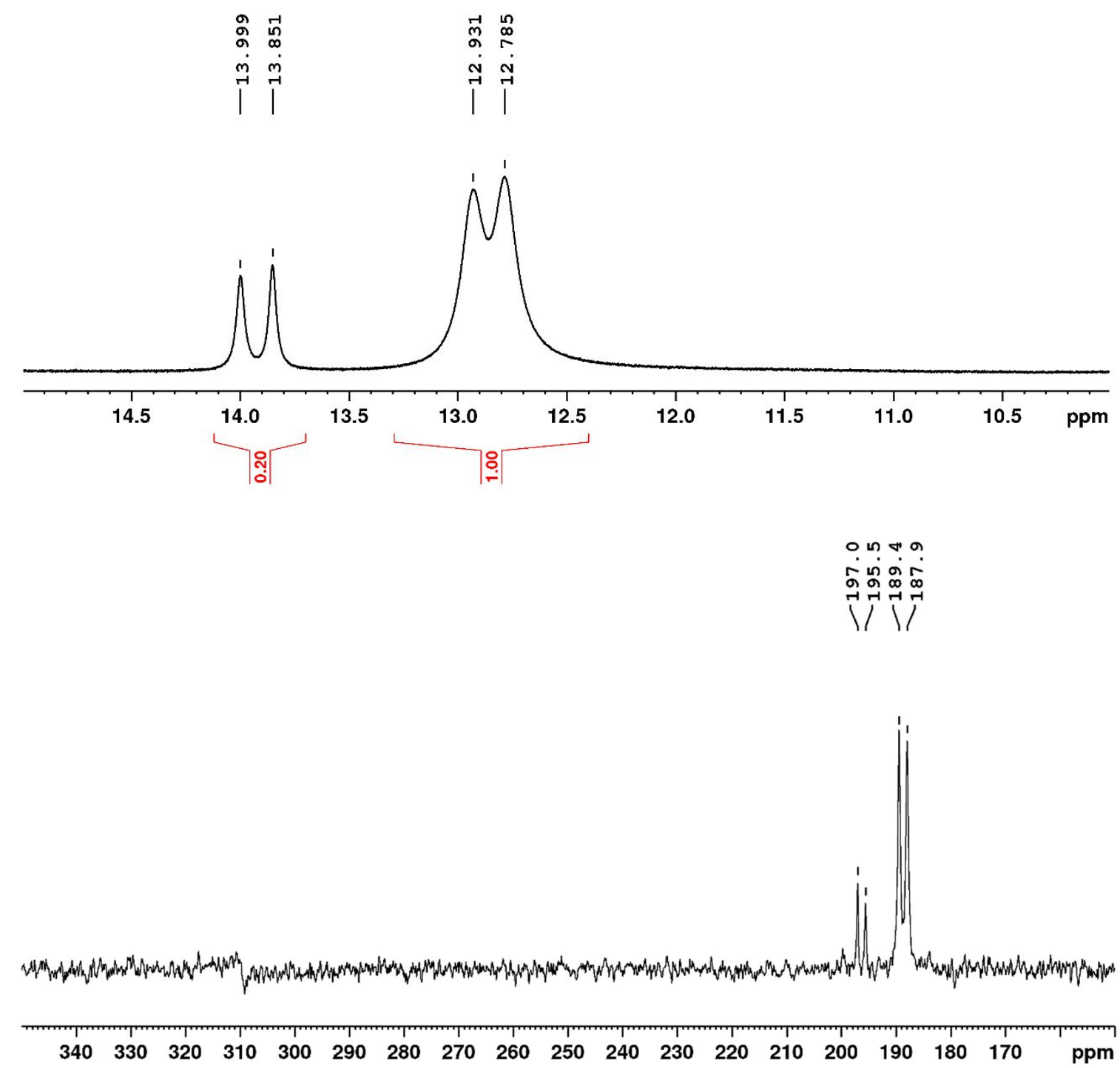

Figure S 1: Low field section of the ${ }^{1} \mathrm{H}$ spectrum $(B F 1=600.0 \mathrm{MHz} ; z g 30 ; \mathrm{NS}=32 ; \mathrm{DS}=0 ; \mathrm{TD}=65536 ; \mathrm{D} 1=1 ; \mathrm{SW}=$ 26.04; O1P = 12) and the ${ }^{15} \mathrm{~N}$ spectrum (BF1 = 60.8 MHz; zg30; NS = 1024; $\mathrm{DS}=0$; $\mathrm{TD}=65536 ; \mathrm{SW}=507.5 \mathrm{ppm} ; \mathrm{O} 1 \mathrm{P}$ $=200)$ of the $\left(\mathrm{CF}_{3}\right)_{2}-\mathrm{DSI} 1 \mathrm{e} / 2 \mathrm{a}-$-complex in $\mathrm{CD}_{2} \mathrm{Cl}_{2}$ at $180 \mathrm{~K}$. 


\subsection{2 $\mathrm{CF}_{3}-\mathrm{DSI} 1 \mathrm{f} / 2 \mathrm{a}\left(\mathrm{CD}_{2} \mathrm{Cl}_{2}, 180 \mathrm{~K}\right)$}
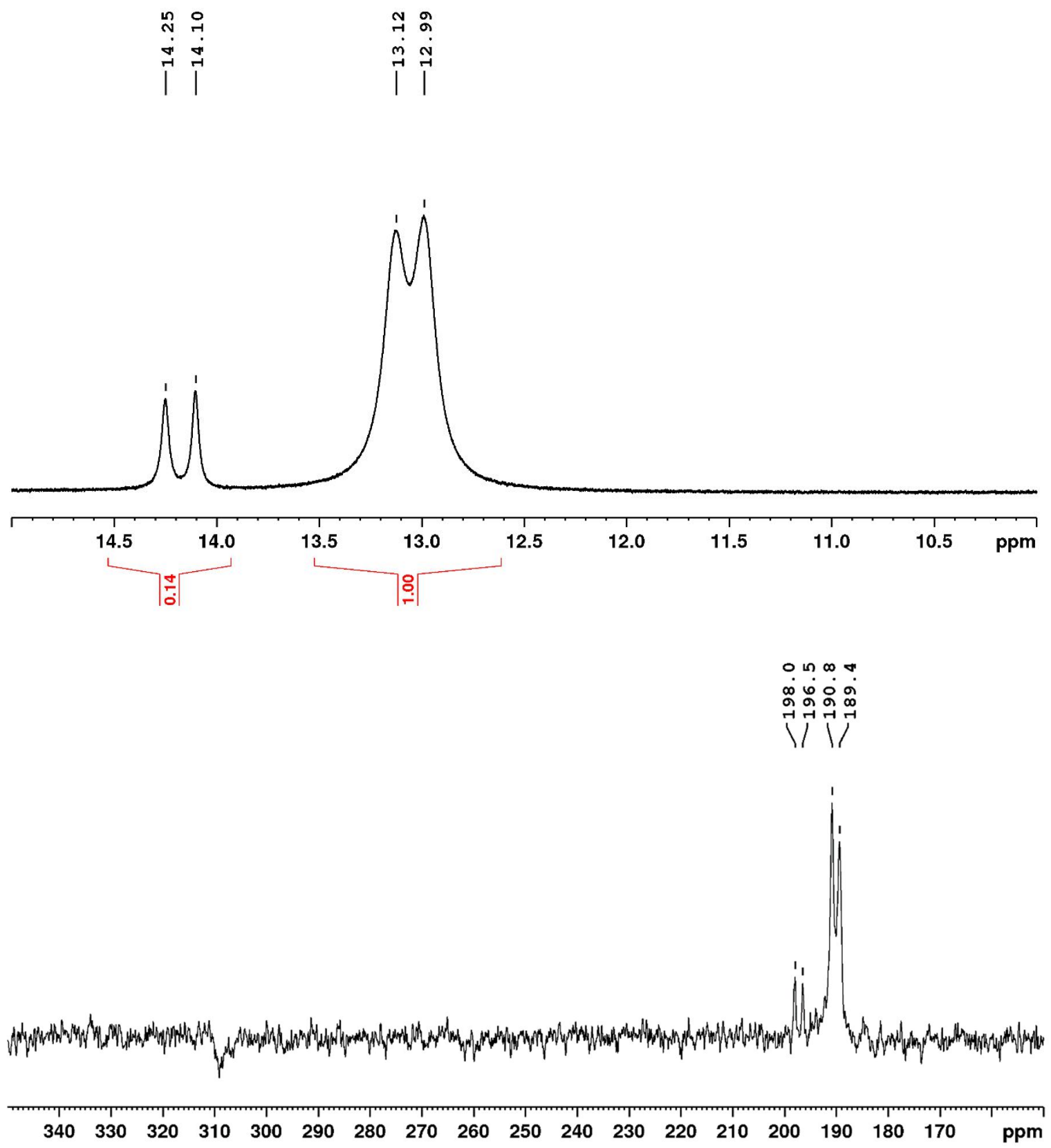

Figure S 2: Low field section of the ${ }^{1} \mathrm{H}$ spectrum $(B F 1=600.0 \mathrm{MHz} ; z g 30 ; \mathrm{NS}=32 ; \mathrm{DS}=0$; TD = 65536; $\mathrm{D} 1=1$; $\mathrm{SW}=$ 26.04; O1P = 12) and the ${ }^{15} \mathrm{~N}$ spectrum (BF1 = 60.8 MHz; zg30; NS = 1024; $\mathrm{DS}=0$; TD = 65536; SW = 507.5 ppm; O1P $=200$ ) of the $\mathrm{CF}_{3}$-DSI 1f/2a-complex in $\mathrm{CD}_{2} \mathrm{Cl}_{2}$ at $180 \mathrm{~K}$. 


\subsection{3 $\left(\mathrm{CF}_{3}\right)_{2}$-DSI 1e/2b $\left(\mathrm{CD}_{2} \mathrm{Cl}_{2}, 180 \mathrm{~K}\right)$}
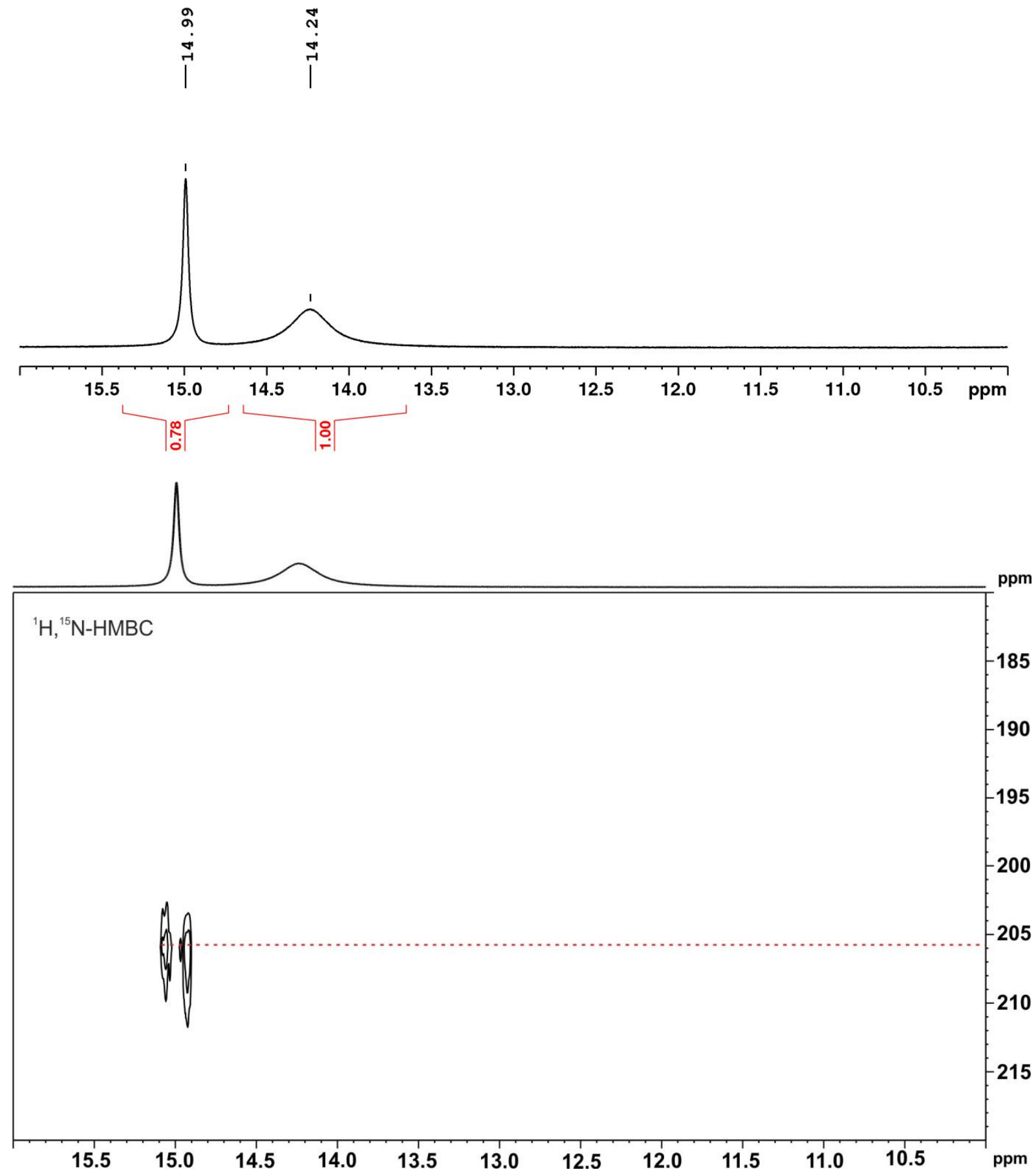

Figure S 3: Low field section of the ${ }^{1} \mathrm{H}$ spectrum $(\mathrm{BF} 1=600.0 \mathrm{MHz} ; z g 30 ; \mathrm{NS}=32 ; \mathrm{DS}=0 ; \mathrm{TD}=65536 ; \mathrm{D} 1=1$; $\mathrm{SW}=$ 26.04; O1P = 12) and the ${ }^{1} \mathrm{H},{ }^{15} \mathrm{~N}-\mathrm{HMBC}$ spectrum (BF1 = $600.0 \mathrm{MHz} ; \mathrm{BF} 2=60.8 \mathrm{MHz}$; inv4gplrndqf_swfix; NS = 32; DS = 16; TD-F2 = 4096; TD-F1 = 128; D1 = 4; SW-F2 = 22.04 ppm; SW-F1 = 499.9 ppm; O1P-F2 = 10; O1P-F1 = 200) of the $\left(\mathrm{CF}_{3}\right)_{2}$-DSI 1e/2b-complex in $\mathrm{CD}_{2} \mathrm{Cl}_{2}$ at $180 \mathrm{~K}$. 


\subsection{4 $\mathrm{CF}_{3}-\mathrm{DSI} 1 \mathrm{f} / 2 \mathrm{~b}\left(\mathrm{CD}_{2} \mathrm{Cl}_{2}, 180 \mathrm{~K}\right)$}
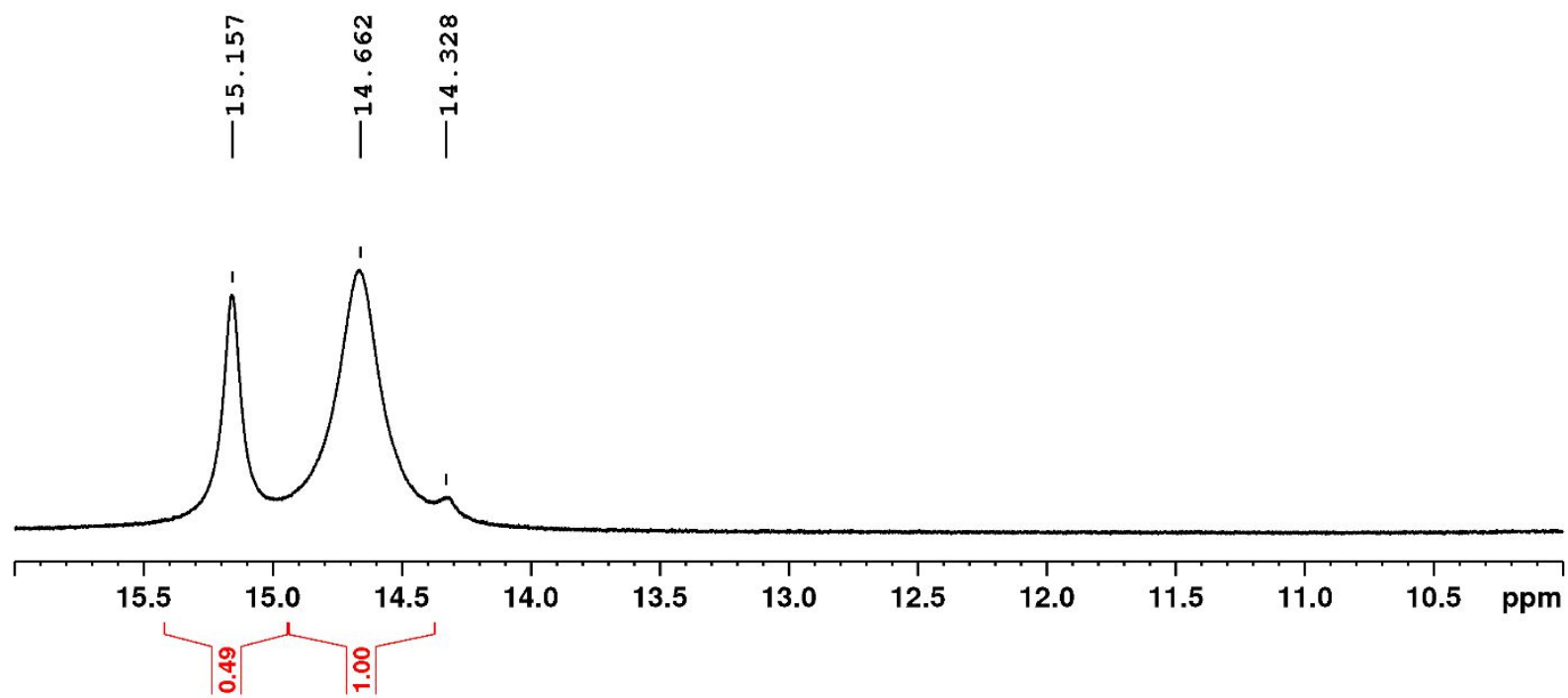

Figure S 4: Low field section of the ${ }^{1} \mathrm{H}$ spectrum $(\mathrm{BF} 1=600.0 \mathrm{MHz} ; z g 30 ; \mathrm{NS}=32 ; \mathrm{DS}=0 ; \mathrm{TD}=65536 ; \mathrm{D} 1=1 ; \mathrm{SW}=$ 26.04; O1P = 12) of the $\mathrm{CF}_{3}$-DSI 1f/2b-complex in $\mathrm{CD}_{2} \mathrm{Cl}_{2}$ at $180 \mathrm{~K}$. 


\section{$1.2 .5\left(\mathrm{CF}_{3}\right)_{2}-\mathrm{DSI} 1 \mathrm{e} / 2 \mathrm{~d}\left(\mathrm{CD}_{2} \mathrm{Cl}_{2}, 180 \mathrm{~K}\right)$}
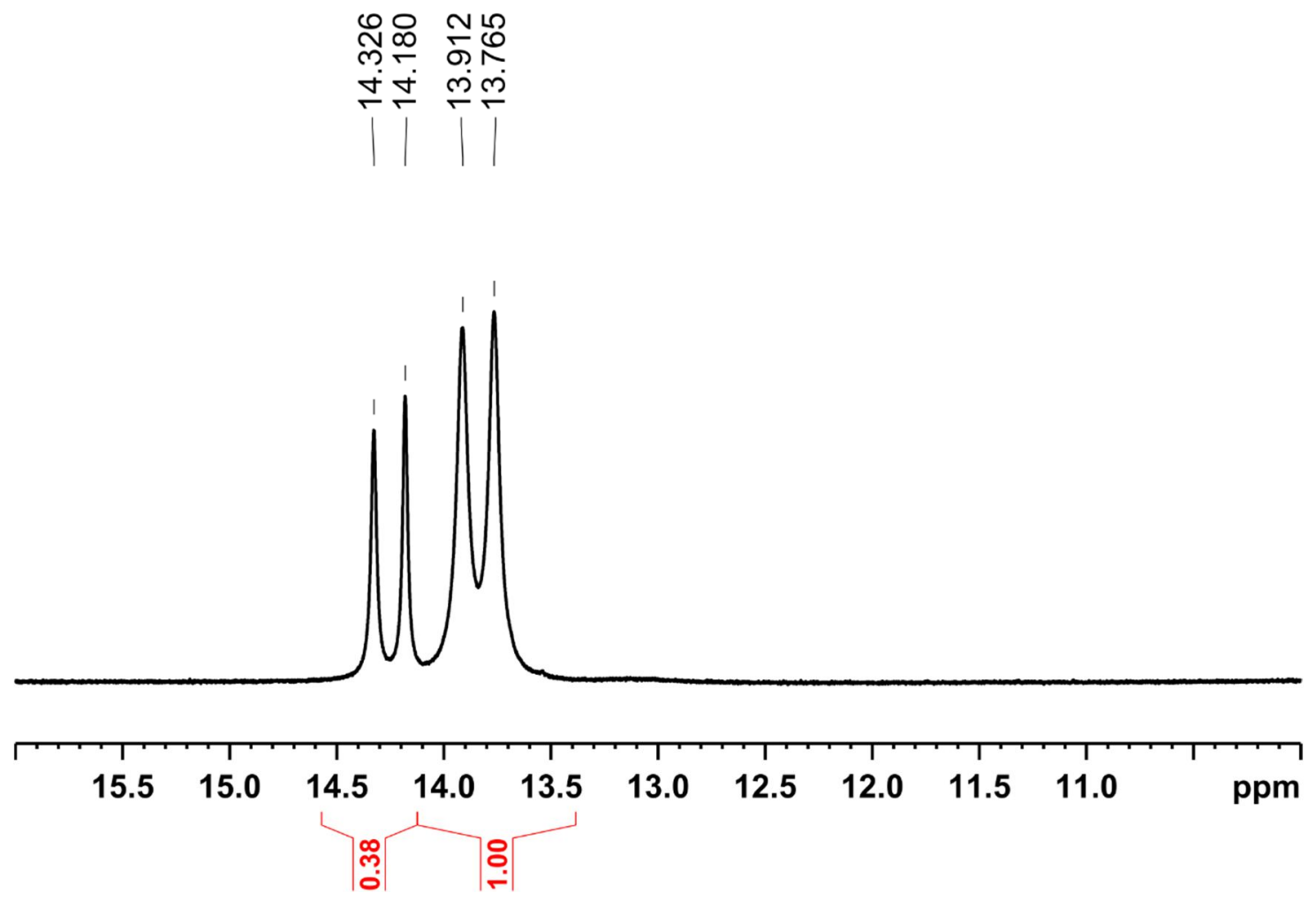

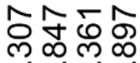

กั่จุ๐

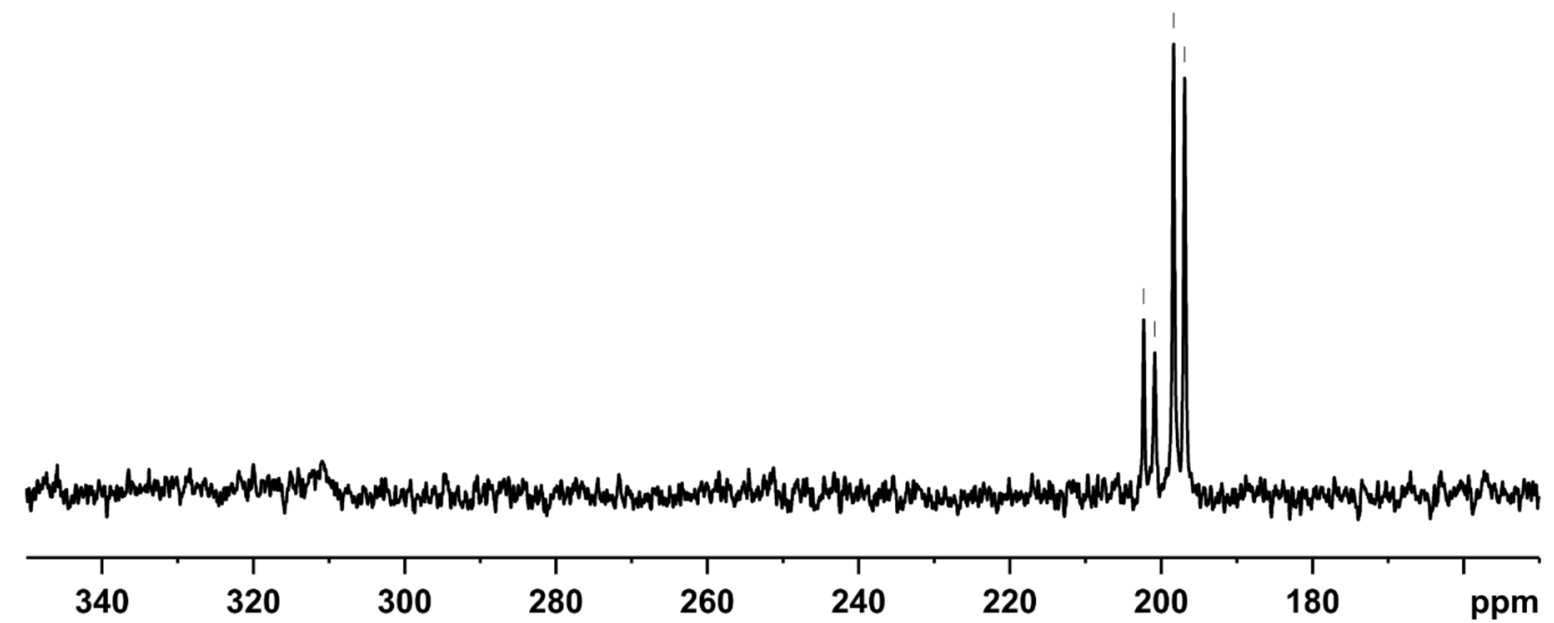

Figure S 5: Low field section of the ${ }^{1} \mathrm{H}$ spectrum $(\mathrm{BF} 1=600.0 \mathrm{MHz} ; z g 30 ; \mathrm{NS}=32 ; \mathrm{DS}=0$; TD = 65536; $\mathrm{D} 1=2 ; \mathrm{SW}=$ $21.55 ; \mathrm{O} 1 \mathrm{P}=8.75)$ and the ${ }^{15} \mathrm{~N}$ spectrum $(\mathrm{BF} 1=60.8 \mathrm{MHz} ; \mathrm{zg} 30 ; \mathrm{NS}=1024 ; \mathrm{DS}=0 ; \mathrm{TD}=65536 ; \mathrm{SW}=507.5 \mathrm{ppm}$; $\mathrm{O} 1 \mathrm{P}=200$ ) of the $\mathrm{CF}_{3}$-DSI $1 \mathbf{e} / \mathbf{2 d}$-complex in $\mathrm{CD}_{2} \mathrm{Cl}_{2}$ at $180 \mathrm{~K}$. 


\subsection{6 $\left(\mathrm{CF}_{3}\right)_{2}$-DSI 1e/3a $\left(\mathrm{CD}_{2} \mathrm{Cl}_{2}, 180 \mathrm{~K}\right)$}
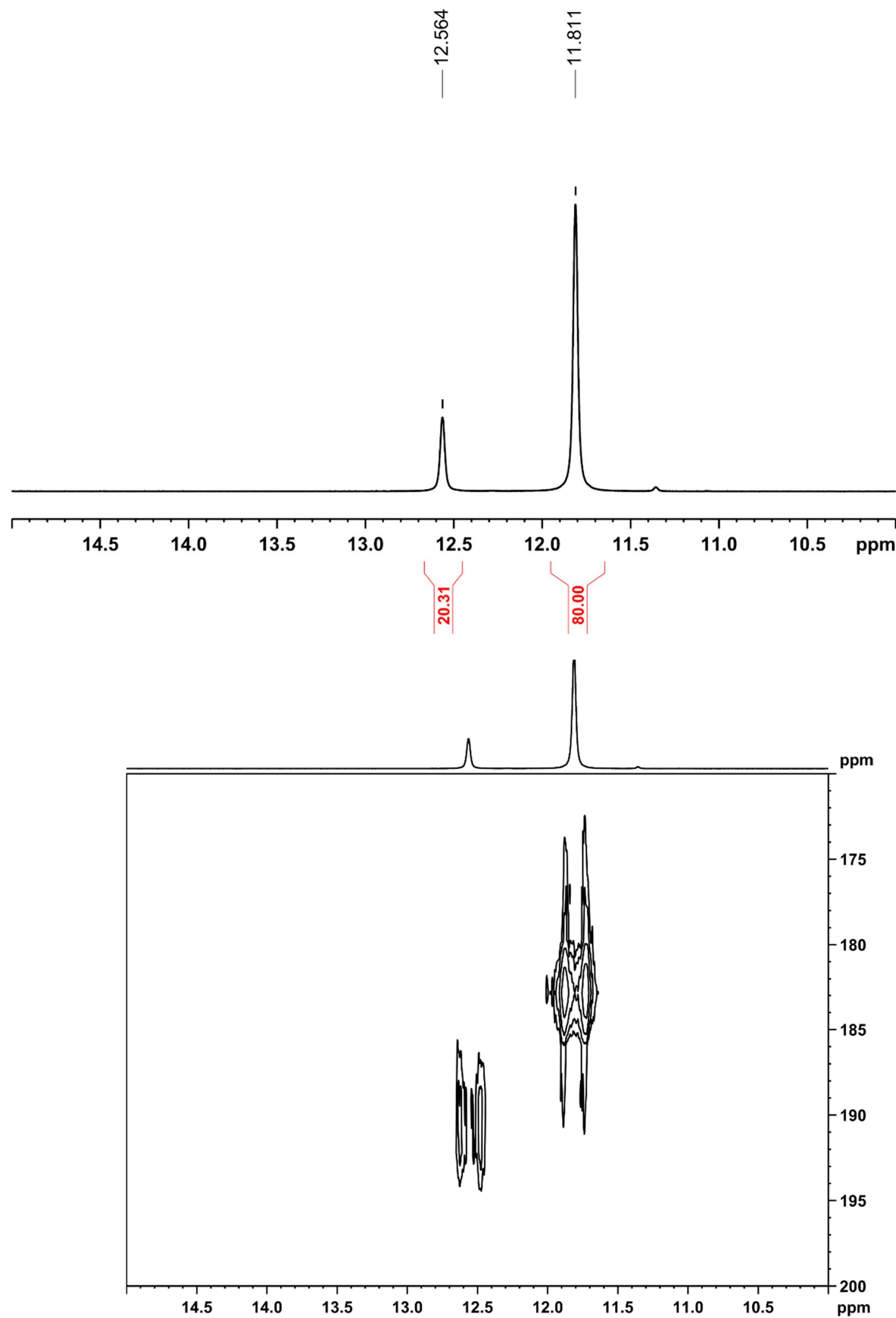

Figure S 6: Low field section of the ${ }^{1} \mathrm{H}$ spectrum $(\mathrm{BF} 1=600.0 \mathrm{MHz} ; z g 30 ; \mathrm{NS}=64 ; \mathrm{DS}=0 \mathrm{TD}=32892 ; \mathrm{D} 1=2 ; \mathrm{SW}=$ 24.03; O1P = 6) and the ${ }^{1} \mathrm{H},{ }^{15} \mathrm{~N}-\mathrm{HMBC}$ spectrum (BF1 $=600.0 \mathrm{MHz} ; \mathrm{BF} 2=60.8 \mathrm{MHz}$; inv4gp/rndqf swfix; NS = 64; DS = 16; TD-F2 = 4096; TD-F1 = 128; D1 = 6; SW-F2 = 14 ppm; SW-F1 = 400 ppm; O1P-F2 = 6.36; O1P-F1 = 200) of the $\left(\mathrm{CF}_{3}\right)_{2}$-DSI 1e/3a (non- ${ }^{15} \mathrm{~N}$-labelled)-complex in $\mathrm{CD}_{2} \mathrm{Cl}_{2}$ at $180 \mathrm{~K}$. 


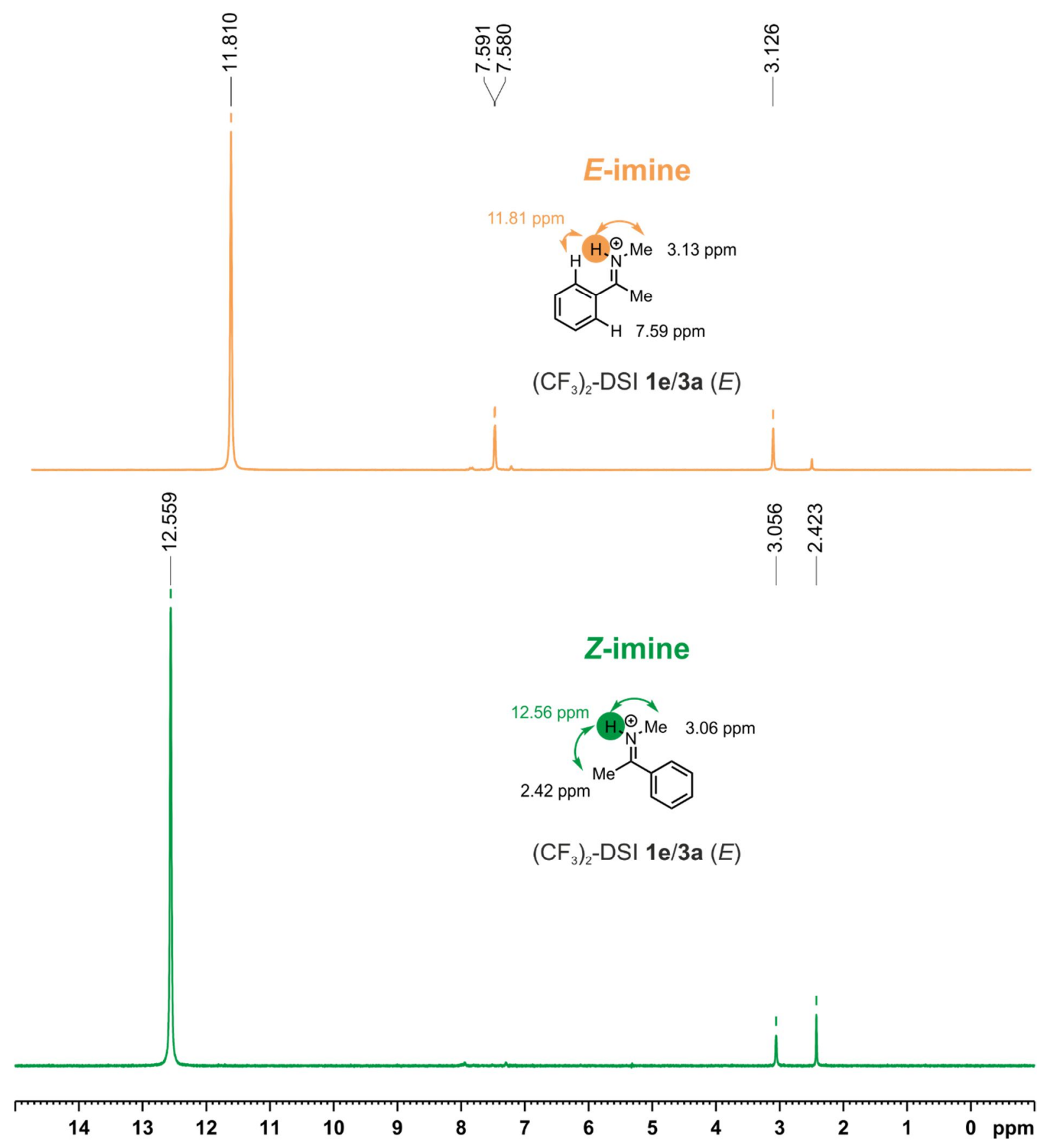

Figure S 7: $1 \mathrm{D}$ selective ${ }^{1} \mathrm{H}$ NOESY spectra $(\mathrm{BF} 1=600.0 \mathrm{MHz}$; selnogp; $\mathrm{NS}=128 ; \mathrm{DS}=2 ; \mathrm{TD}=65536 ; \mathrm{D} 1=2 ; \mathrm{SW}=$ $24 \mathrm{ppm} ; \mathrm{O} 1 \mathrm{P}=6$, mixing time $\mathrm{D} 8=150 \mathrm{~ms}$ ) of the $\left(\mathrm{CF}_{3}\right)_{2}$-DSI 1e/3a (non- ${ }^{-15} \mathrm{~N}-$ labelled)-complex in $\mathrm{CD}_{2} \mathrm{Cl}_{2}$ at $180 \mathrm{~K}$ irradiated at $\delta 11.81 \mathrm{ppm}$ (top spectrum) and $12.56 \mathrm{ppm}$ (bottom spectrum). 


\subsubsection{TRIP 1a/3a $\left(\mathrm{CD}_{2} \mathrm{Cl}_{2}, 180 \mathrm{~K}\right)$}

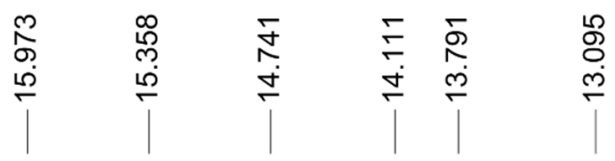
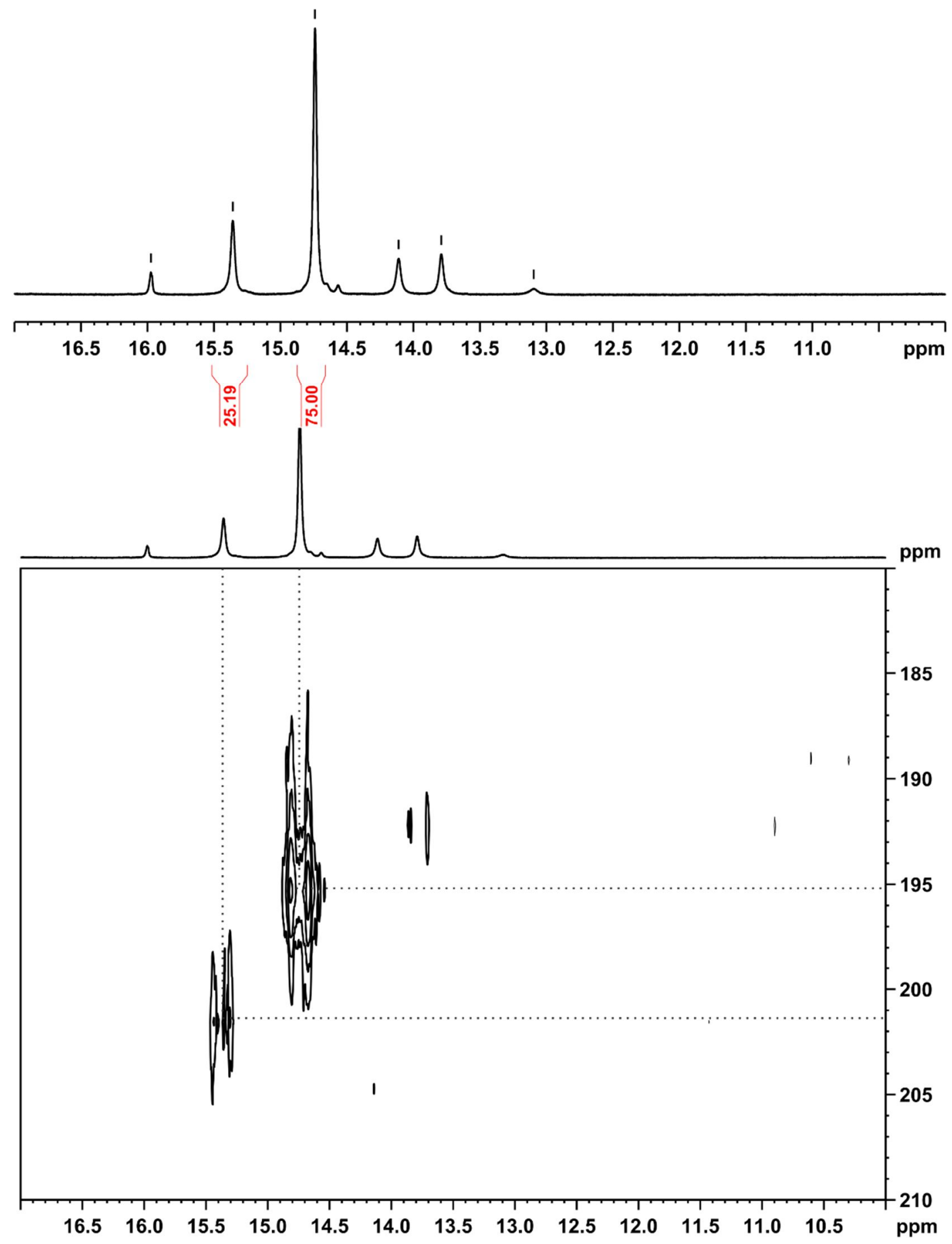

Figure S 8: Low field section of the ${ }^{1} \mathrm{H}$ spectrum $(\mathrm{BF} 1=600.0 \mathrm{MHz} ; \mathrm{zg} 30 ; \mathrm{NS}=64 ; \mathrm{DS}=0 \mathrm{TD}=32892 ; \mathrm{D} 1=2 ; \mathrm{SW}=$ 24.03; O1P = 6) and the ${ }^{1} \mathrm{H},{ }^{15} \mathrm{~N}-\mathrm{HMBC}$ spectrum (BF1 $=600.0 \mathrm{MHz} ; \mathrm{BF} 2=60.8 \mathrm{MHz}$; inv4gplrndqf_swfix; NS = 64; DS = 16; TD-F2 = 4096; TD-F1 = 128; D1 = 6; SW-F2 = 18 ppm; SW-F1 = 400 ppm; O1P-F2 = 7.98; O1P-F1 = 200) of the TRIP 1a/2e (non- ${ }^{15} \mathrm{~N}$-labelled)-complex in $\mathrm{CD}_{2} \mathrm{Cl}_{2}$ at $180 \mathrm{~K}$. 


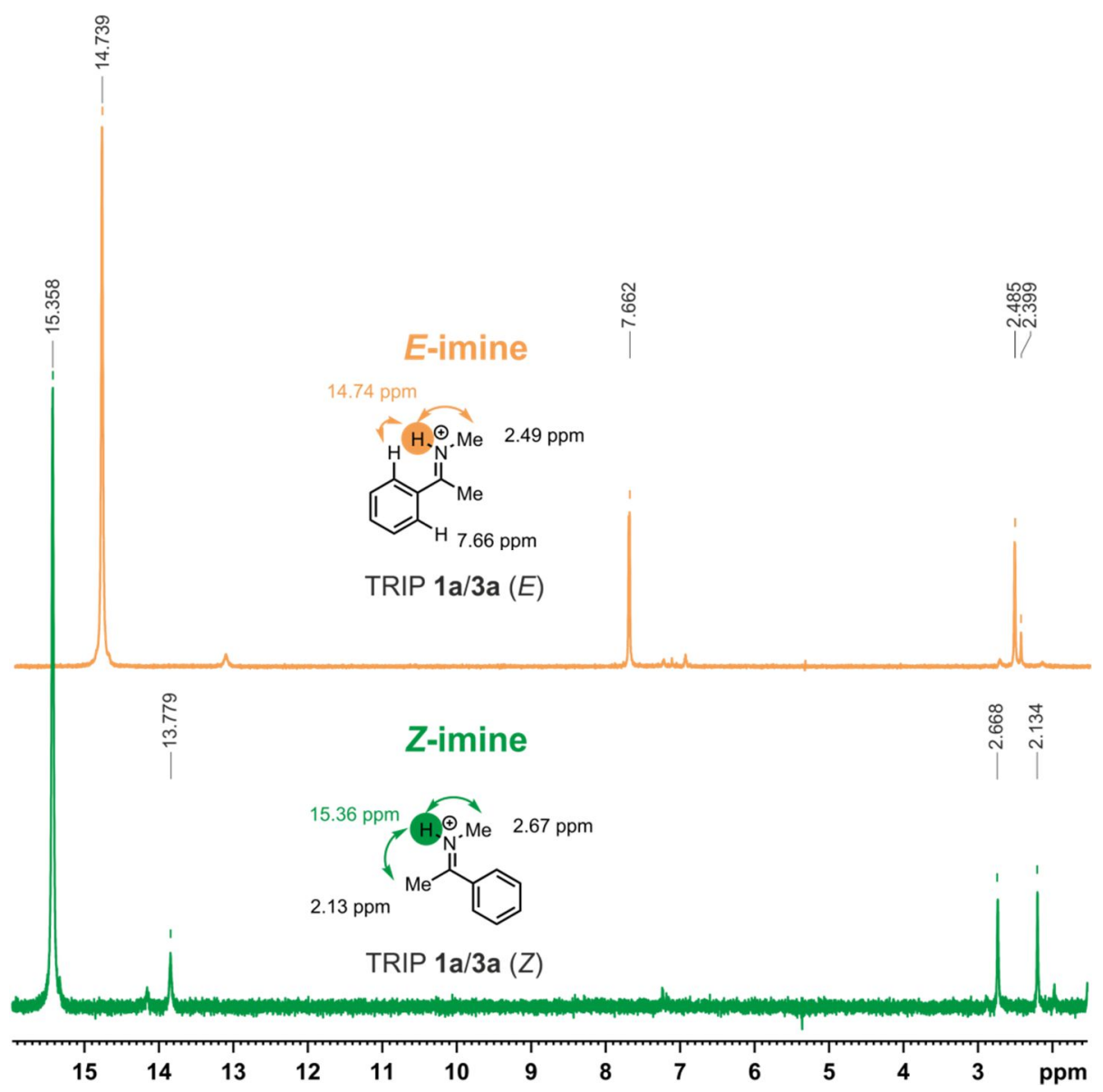

Figure S 9: ${ }^{1} \mathrm{H} 1 \mathrm{D}$ selective NOESY spectra $(\mathrm{BF} 1=600.0 \mathrm{MHz}$; selnogp; $\mathrm{NS}=128 ; \mathrm{DS}=2 ; \mathrm{TD}=65536 ; \mathrm{D} 1=2 ; \mathrm{SW}=$ $24 \mathrm{ppm} ; \mathrm{O} 1 \mathrm{P}=6$, mixing time D8 $=150 \mathrm{~ms}$ ) of the TRIP $1 \mathbf{a} / 3 \mathbf{a}$ (non- ${ }^{15} \mathrm{~N}$-labelled)-complex in $\mathrm{CD}_{2} \mathrm{Cl}_{2}$ at $180 \mathrm{~K}$ irradiated at $\delta 14.74 \mathrm{ppm}$ (top spectrum) and $15.36 \mathrm{ppm}$ (bottom spectrum).

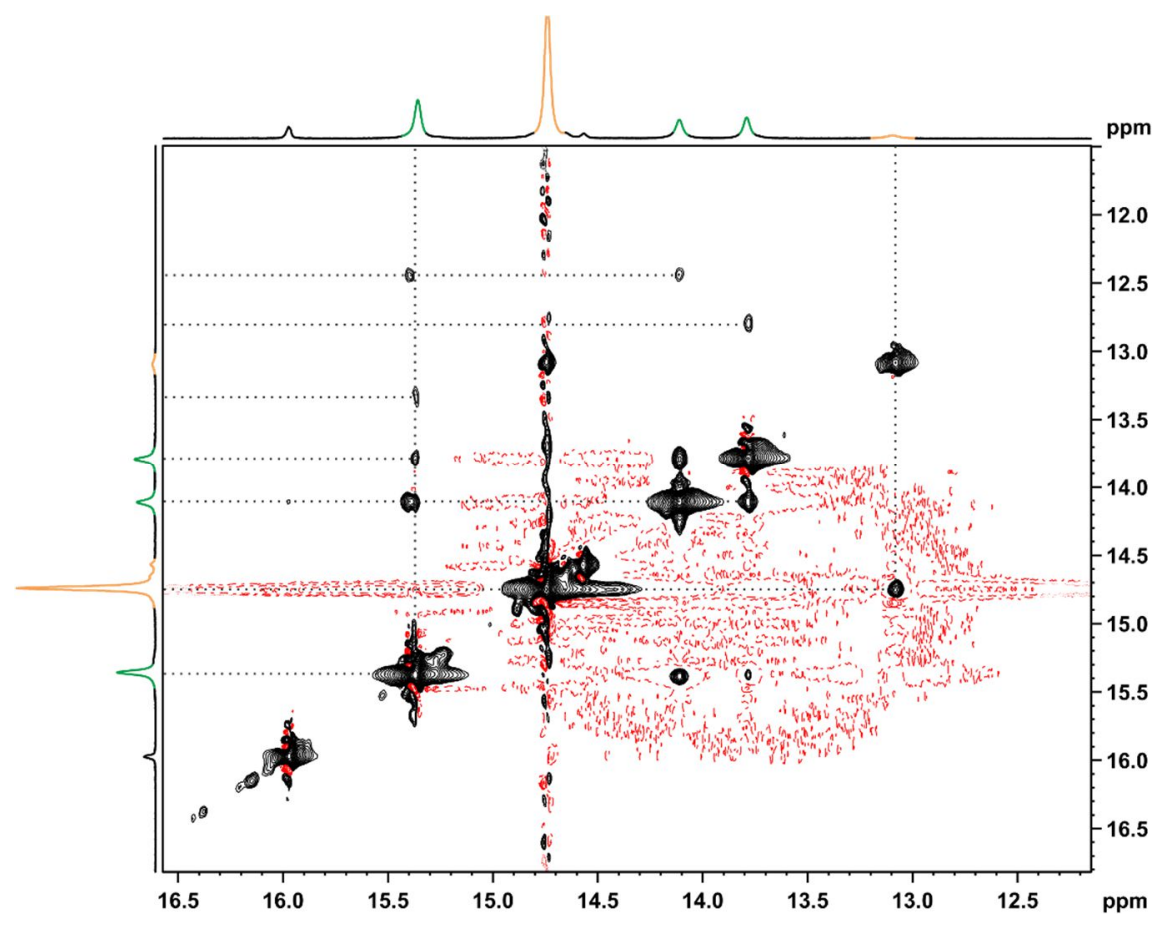

Figure S 10: Low field section of ${ }^{1} \mathrm{H}$ 2D NOESY spectrum (BF1 = 600.0 MHz; noeygpph; NS = 24; DS = 16; TD1 = 256; $\mathrm{D} 1=4 ; \mathrm{SW}=18 \mathrm{ppm} ; \mathrm{O} 1 \mathrm{P}=8$, mixing time D8 $=100 \mathrm{~ms}$ ) of the TRIP $1 \mathrm{a} / 3 \mathrm{a}$ (non- ${ }^{15} \mathrm{~N}$-labelled)-complex in $\mathrm{CD}_{2} \mathrm{Cl}_{2}$ at $180 \mathrm{~K}$, showing the presence of various $E$ - and $Z$-complexes. 


\subsubsection{BINSA 1g/2e $\left(\mathrm{CD}_{2} \mathrm{Cl}_{2}, 180 \mathrm{~K}\right)$}

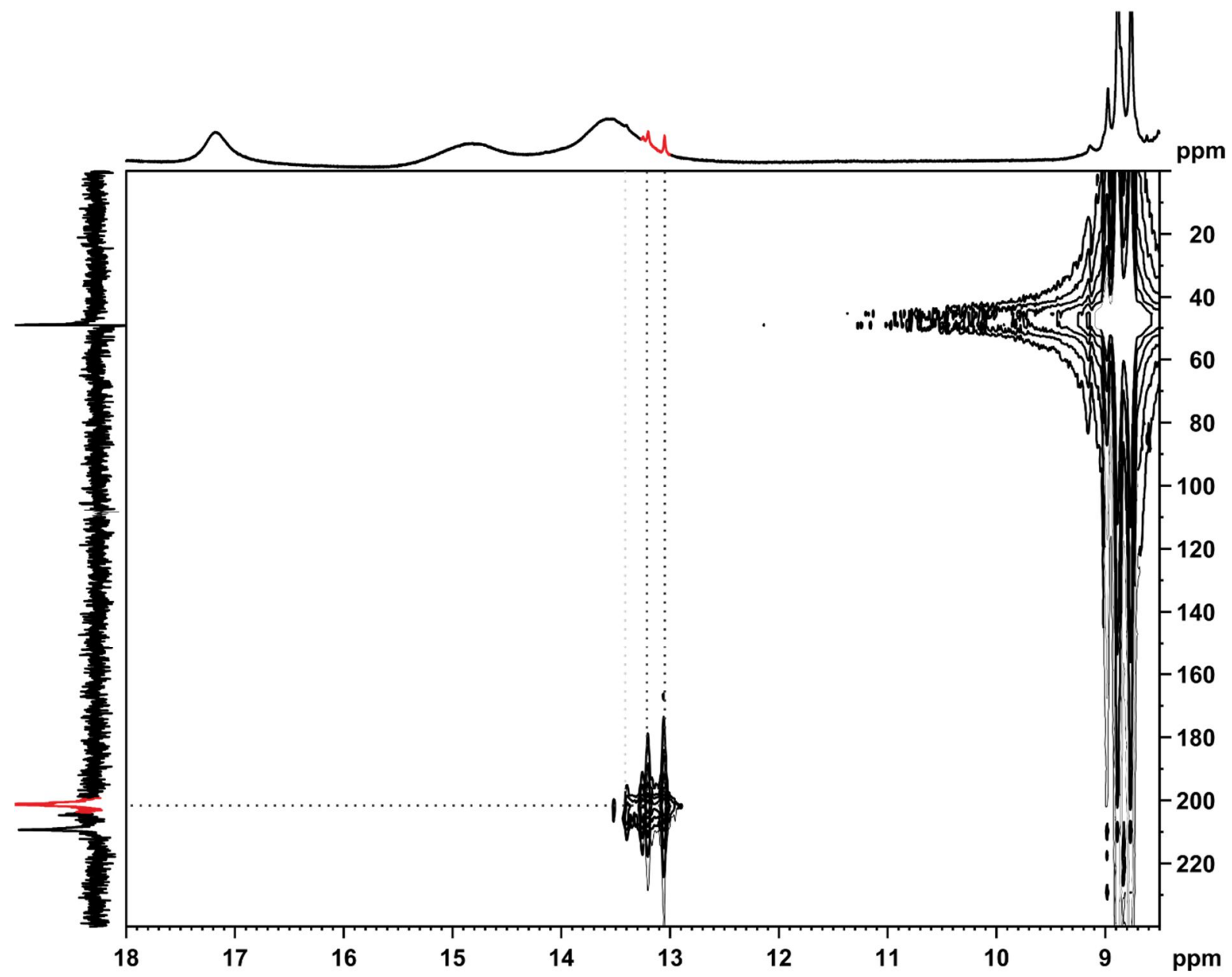

Figure S 11: Low field section of the ${ }^{1} \mathrm{H}$ spectrum (BF1 = $600.0 \mathrm{MHz} ; z g 30 ; \mathrm{NS}=64 ; \mathrm{DS}=0 \mathrm{TD}=32892 ; \mathrm{D} 1=2$; $\mathrm{SW}=$ 22; O1P = 10), ${ }^{15} \mathrm{~N}$ spectrum $(\mathrm{BF} 1=60.8 \mathrm{MHz} ; z g 30 ; \mathrm{NS}=2048 ; \mathrm{DS}=0 ; \mathrm{TD}=65536 ; \mathrm{SW}=507.5 \mathrm{ppm} ; \mathrm{O} 1 \mathrm{P}=200)$ and the ${ }^{1} \mathrm{H},{ }^{15} \mathrm{~N}-\mathrm{HMBC}$ spectrum $(\mathrm{BF} 1=600.0 \mathrm{MHz} ; \mathrm{BF} 2=60.8 \mathrm{MHz} ;$ inv4gplrndqf_swfix; NS = 16; DS = 16; TD-F2 = 4096; TD-F1 = 128; D1 = 6; SW-F2 = 22 ppm; SW-F1 = 500 ppm; O1P-F2 = 10; O1P-F1 = 200) of the BINSA 1g/2ecomplex in $\mathrm{CD}_{2} \mathrm{Cl}_{2}$ at $180 \mathrm{~K}$. 


\section{Steiner-Limbach Correlation}

\subsection{Theory}

For the theory behind the hydrogen bond analysis of the chemical shifts using the SteinerLimbach curve see the supporting material of the reference. ${ }^{[1,2]}$

\subsection{Used fit parameters for the Steiner-Limbach curve}

Table S 2: Used NMR parameters $\left(\delta(\underline{N})^{\circ}, \delta(H \underline{N})^{\circ}, \delta(O H \underline{N})^{*}, \delta(O \underline{H})^{\circ}, \delta(\underline{H} N)^{\circ}, \delta(O \underline{H} N)^{*}\right)$ as well as the correction factors $\left(\mathrm{f}, \mathrm{g}, \mathrm{c}^{\mathrm{H}}, \mathrm{d}^{\mathrm{H}}\right)$ used for the fit of the Steiner-Limbach curve are shown. They were taken from reference ${ }^{[2]}$ and slightly modified to our initial study of TRIP/imine-complexes. ${ }^{[1]}$

\begin{tabular}{|c|c|c|c|c|c|c|c|c|c|c|}
\hline systems & $\begin{array}{c}\delta(\underline{N})^{\circ} \\
{[p p m]}\end{array}$ & $\begin{array}{l}\delta(\mathrm{HN})^{\circ} \\
{[\mathrm{ppm}]}\end{array}$ & $\begin{array}{c}\delta(\mathrm{OHN})^{*} \\
{[\mathrm{ppm}]}\end{array}$ & $\begin{array}{l}\delta(\mathrm{OH})^{\circ} \\
{[\mathrm{ppm}]}\end{array}$ & $\begin{array}{l}\delta(\underline{\mathrm{HN}})^{\circ} \\
{[\mathrm{ppm}]}\end{array}$ & $\begin{array}{c}\delta(\mathrm{OHN})^{*} \\
{[\mathrm{ppm}]}\end{array}$ & f & g & $\mathrm{c}^{\mathrm{H}}$ & $d^{H}$ \\
\hline imine/TRIP[1] & 0 & -170 & -6 & 2 & 6 & 16 & 8 & 2 & 360 & 0.3 \\
\hline $\begin{array}{l}\text { imine/TiPSY } \\
\text { imine/TRIFP } \\
\text { imine/TRIM }^{[2]}\end{array}$ & 0 & -151.6 & -4.89 & 1.04 & 12.33 & 14.2 & 7.3 & 1.36 & 349.9 & 0.33 \\
\hline imine /DSI & 0 & -151.6 & -4.89 & 1.04 & 12.33 & 14.2 & 7.3 & 1.36 & 349.9 & 0.33 \\
\hline
\end{tabular}

\subsection{Referencing of the ${ }^{15} \mathrm{~N}$ chemical shift}

As proposed in literature the ${ }^{15} \mathrm{~N}$ chemical shifts were referenced to the chemical shift of the non-hydrogen bonded imine ${ }^{15} \mathrm{~N}$-labelded $(E)-1$-(4-bromophenyl)- $N$-phenylethan-1-imine $\left(\delta^{15} \mathrm{~N}=340.8 \mathrm{ppm}^{[1]}, 300 \mathrm{~K}, \mathrm{CD}_{2} \mathrm{Cl}_{2}\right) .{ }^{[3]}$ Thus, the ${ }^{15} \mathrm{~N}$ chemical shifts reflect the deviation of the complexed imines from their neutral form $\left(\delta^{15} \mathrm{~N}=\sim 330 \mathrm{ppm}\right)$ and range from -90 to -150 ppm. Because of this correction $\delta(\underline{\mathrm{N}})^{\circ}$ can be set to 0 . 


\subsection{Steiner-Limbach curve with TRIP/3a-, $\left(\mathrm{CF}_{3}\right)_{2}$-DSI/3a- and BINSA/2e-complexes}

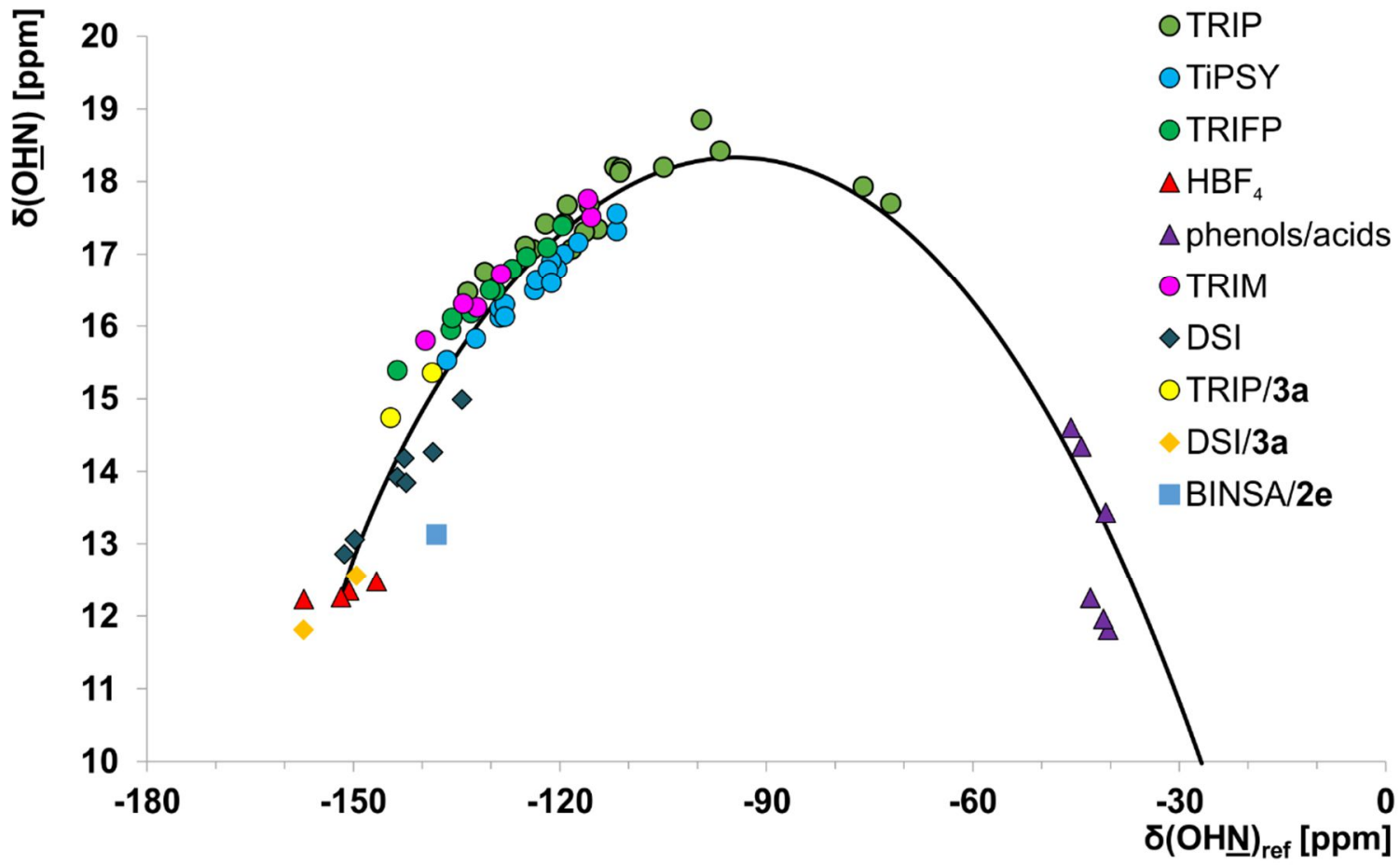

Figure S 12: Steiner-Limbach correlation curve with experimental $\delta{ }^{1} \mathrm{H}$ and $\delta{ }^{15} \mathrm{~N}$ chemical shifts of the imine complexes. The curve shows data for complexes with weak acids, chiral phosphoric acids, disulfonimides, disulfonic acid, and $\mathrm{HBF}_{4}$. 


\section{$3 \quad{ }^{15} \mathrm{~N}$-labeling of the DSI $1 \mathrm{e}$}

\subsection{Synthesis of the DSI-precursor BINSA}

The disulfonic acid BINSA (19) was synthesized according to the literature. ${ }^{[4]}$<smiles>Oc1c(-c2cc(C(F)(F)F)cc(C(F)(F)F)c2)cc2ccccc2c1-c1c(O)c(-c2cc(C(F)(F)F)cc(C(F)(F)F)c2)cc2ccccc12</smiles>

5

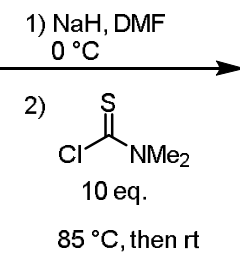

$85^{\circ} \mathrm{C}$, then $\mathrm{rt}$

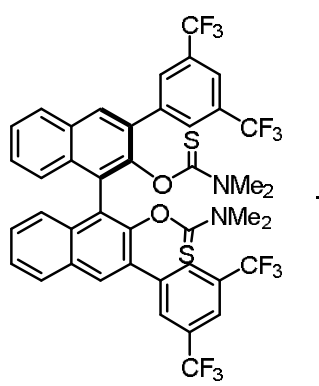

6
$97 \%$ yield<smiles>COC(=O)Sc1c(-c2cc(C(F)(F)F)cc(C(F)(F)F)c2)cc2ccccc2c1-c1c(-c2cc(C(F)(F)F)cc(C(F)(F)F)c2)cc2ccccc2c1-c1cc(C(F)(F)F)cc(C(F)(F)F)c1</smiles>

$79 \%$ yield<smiles>O=C(O)c1c(-c2cc(C(F)(F)F)cc(C(F)(F)F)c2)cc2ccccc2c1-c1c(O)c(-c2cc(C(F)(F)F)cc(C(F)(F)F)c2)cc2ccccc12</smiles>

$1 \mathrm{~g}$
$46 \%$ yield

\subsection{Synthesis of the ${ }^{15} \mathrm{~N}$-labeled DSI 1e}

The ${ }^{15} \mathrm{~N}$-DSI 1e was synthesized from its precursor BINSA using ${ }^{15} \mathrm{NH}_{3}$ (aq.) (instead of $2 \mathrm{M}$ $\mathrm{NH}_{3}$ in $\mathrm{MeOH}$ ) by following the literature procedure. ${ }^{[4]}$

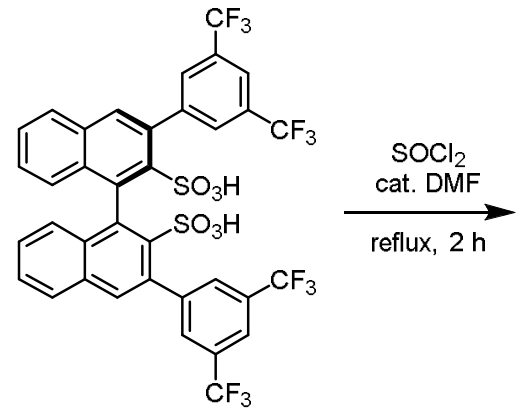

$1 \mathrm{~g}$<smiles>O=S(=O)(OCl)c1c(-c2cc(C(F)(F)F)cc(C(F)(F)F)c2)cc2ccccc2c1-c1cc2ccccc2cc1-c1cc(C(F)(F)F)cc(C(F)(F)F)c1</smiles>

8

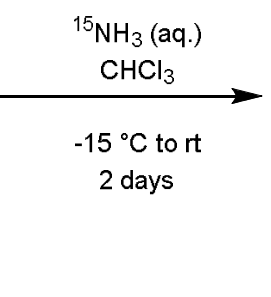

$-15^{\circ} \mathrm{C}$ to $\mathrm{rt}$<smiles>O=S(=O)(O)c1cc2ccccc2cc1S(=O)(=O)c1cc(C(F)(F)F)cc(C(F)(F)F)c1</smiles>

(R)-DSI-1e 


\subsection{Comparison of the spectra of ${ }^{15} \mathrm{~N}$-labeld and not labeled $\left(\mathrm{CF}_{3}\right)_{2}-\mathrm{DSI} 1 \mathrm{e} / 2 \mathrm{a}$ $\left(\mathrm{CD}_{2} \mathrm{Cl}_{2}, 180 \mathrm{~K}\right)$}

a) ${ }^{1} \mathrm{H}$ spectrum

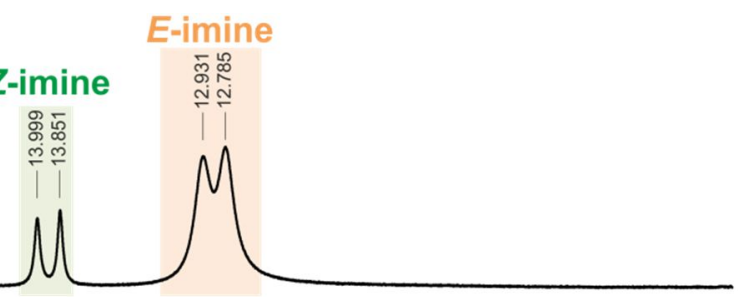

not labeld DSI 1e/2a

b) ${ }^{1} \mathrm{H}$ spectrum

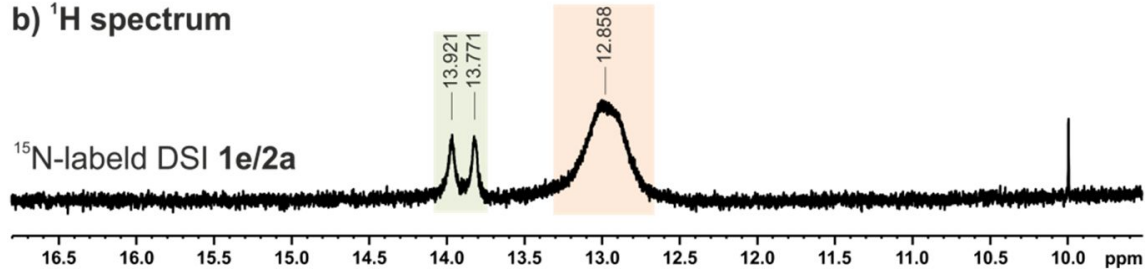

Figure S 13: a) Low field section of the ${ }^{1} \mathrm{H}$ spectrum of DSI 1e and ${ }^{15} \mathrm{~N}$-labeled imine $2 \mathrm{a}(1: 1,50 \mathrm{mM})$ in $\mathrm{CD}_{2} \mathrm{Cl}_{2}$ at $180 \mathrm{~K} . \mathrm{b})$ The region of the hydrogen bonds with in the ${ }^{1} \mathrm{H}$ spectra of ${ }^{15} \mathrm{~N}$-labeld $\left(\mathrm{CF}_{3}\right)_{2}$-DSI $1 \mathrm{e}$ and ${ }^{15} \mathrm{~N}$-labeled imine 2a $(1: 1,32 \mathrm{mM})$ in $\mathrm{CD}_{2} \mathrm{Cl}_{2}$ at $180 \mathrm{~K}$. The signal of the hydrogen bonded proton in the binary $E$-complex is significantly broadened.
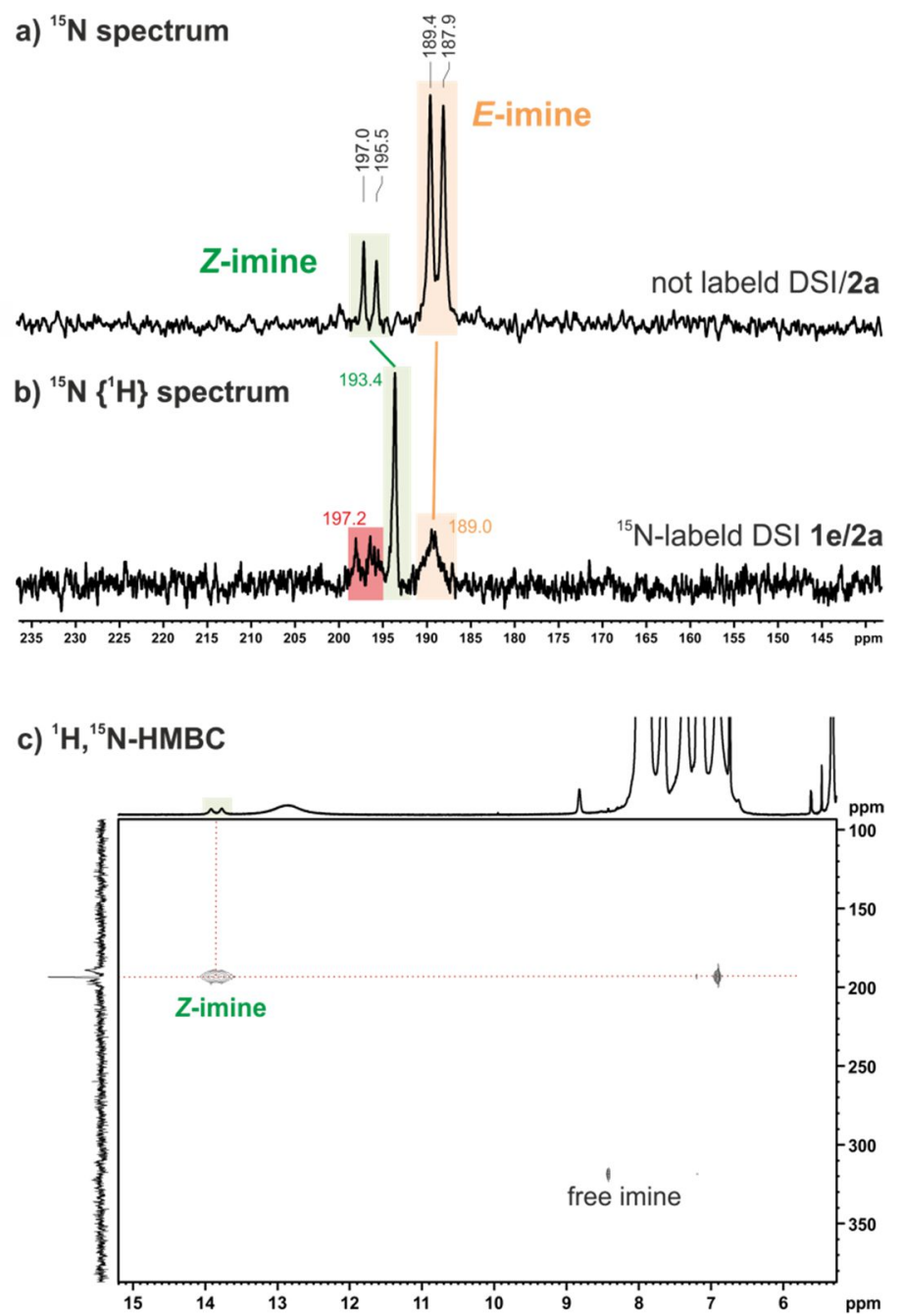

Figure S 14: a) ${ }^{15} \mathrm{~N}$ spectrum of DSI $1 \mathrm{e}$ and ${ }^{15} \mathrm{~N}$-labeled imine $2 \mathrm{a}(1: 1,50 \mathrm{mM})$ in $\mathrm{CD}_{2} \mathrm{Cl}_{2}$ at $180 \mathrm{~K}$. b) To increase the sensitivity of the ${ }^{15} \mathrm{~N}$ spectra an inverse gated decoupled ${ }^{15} \mathrm{~N}$ spectra was recorded of the ${ }^{15} \mathrm{~N}$-labeld DSI $1 \mathrm{e}$ and imine $2 \mathrm{a}(1: 1,32 \mathrm{mM})$ in $\mathrm{CD}_{2} \mathrm{Cl}_{2}$ at $180 \mathrm{~K}$. The red signals may be from the ${ }^{15} \mathrm{~N}$ of the catalyst within the binary $\mathrm{E}$ - and Z-complex. c) To identify the observed signals a ${ }^{1} \mathrm{H},{ }^{15} \mathrm{~N}-\mathrm{HMBC}$ at $180 \mathrm{~K}$ was measured. 


\subsection{Comparison of the ${ }^{15} \mathrm{~N}$-spectra of the free $\left(\mathrm{CF}_{3}\right)_{2}-\mathrm{DSI} 1 \mathrm{e}$ and the $\left(\mathrm{CF}_{3}\right)_{2}-\mathrm{DSI} 1 \mathrm{e} / 2 \mathrm{a}$ - binary complex $\left(\mathrm{CD}_{2} \mathrm{Cl}_{2}\right)$}

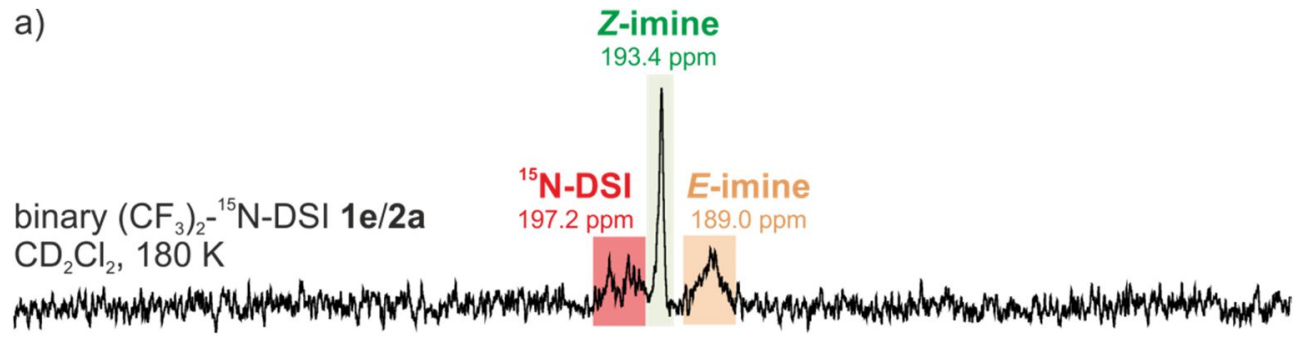

b)

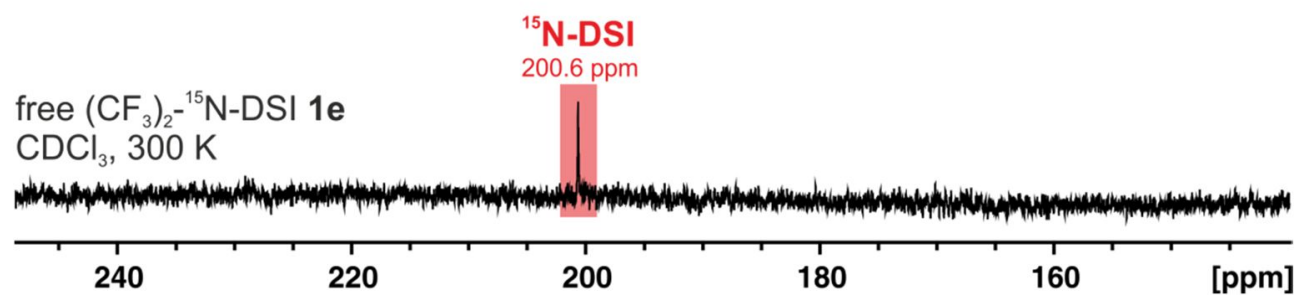

Figure S 15: a) To increase the sensitivity of the ${ }^{15} \mathrm{~N}$ spectrum an inverse gated decoupled ${ }^{15} \mathrm{~N}$ spectrum was recorded of the ${ }^{15} \mathrm{~N}$-labeld DSI $1 \mathrm{e}$ and imine $2 \mathrm{a}(1: 1,32 \mathrm{mM})$ in $\mathrm{CD}_{2} \mathrm{Cl}_{2}$ at $180 \mathrm{~K}$. The red signal, which seems to be from the ${ }^{15} \mathrm{~N}$ of the catalyst within the binary $E$ - and Z-complex, is significantly broadened. b) In contrast the signal of the free ${ }^{15} \mathrm{~N}$-labeled DSI $1 \mathrm{e}$ in an inverse gated decoupled ${ }^{15} \mathrm{~N}$ spectrum is sharp $\left(\mathrm{CDCl}_{3}, 300 \mathrm{~K}\right)$. 


\section{Calculated ${ }^{1} J_{\mathrm{HN}},{ }^{1 \mathrm{~h}} \mathrm{~J}_{\mathrm{NH}}$ and ${ }^{2 \mathrm{~h}} \mathrm{~J}_{\mathrm{NN}}$ Coupling Constants}

For the calculations of the coupling constants of type $E_{N}$, a reduced model (truncated 3,3'substituents) based on the ground state was used. TPSS with IGLO-III basis set were used in the calculation.

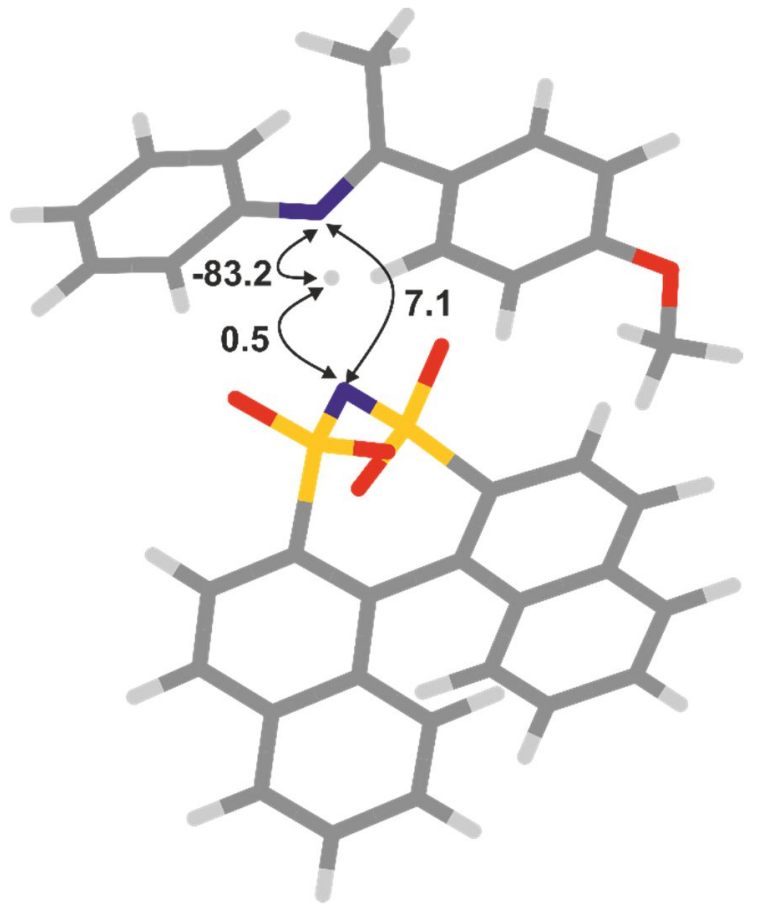

Figure S 16: All calculated coupling constants for the most stable type $E_{N}$ structure of the simplified binary DSI/2a-complex are shown (in Hz). The catalyst structure was simplified by truncation of the 3,3'-substitents. 


\section{Assignments of the ${ }^{1} \mathrm{H}$ and ${ }^{13} \mathrm{C}$ Chemical Shifts}

The ${ }^{1} \mathrm{H}$ (red) and ${ }^{13} \mathrm{C}$ (blue) chemical shifts of all investigated binary complexes were assigned with standard 2D NMR experiments $\left({ }^{1} \mathrm{H},{ }^{1} \mathrm{H}\right.$-COSY, ${ }^{1} \mathrm{H},{ }^{1} \mathrm{H}$-TOCSY, ${ }^{1} \mathrm{H},{ }^{1} \mathrm{H}$-NOESY, ${ }^{1} \mathrm{H},{ }^{13} \mathrm{C}-\mathrm{HSQC},{ }^{1} \mathrm{H},{ }^{13} \mathrm{C}-\mathrm{HMBC}$ ) at $180 \mathrm{~K}$. The ${ }^{15} \mathrm{~N}$ (orange) and ${ }^{19} \mathrm{~F}$ (green) chemical shifts were assigned with ${ }^{1} \mathrm{H},{ }^{19} \mathrm{~F}-\mathrm{HMBC}$ and ${ }^{1} \mathrm{H},{ }^{15} \mathrm{~N}-\mathrm{HMBC}$ spectra respectively.

\section{$5.1 \quad\left(C F_{3}\right)_{2}-D S I$ 1e/2a $\left(C D_{2} C l_{2}, 180 K\right)$}

${ }^{19} \mathrm{~F}:-62,2$ and $-61,9$
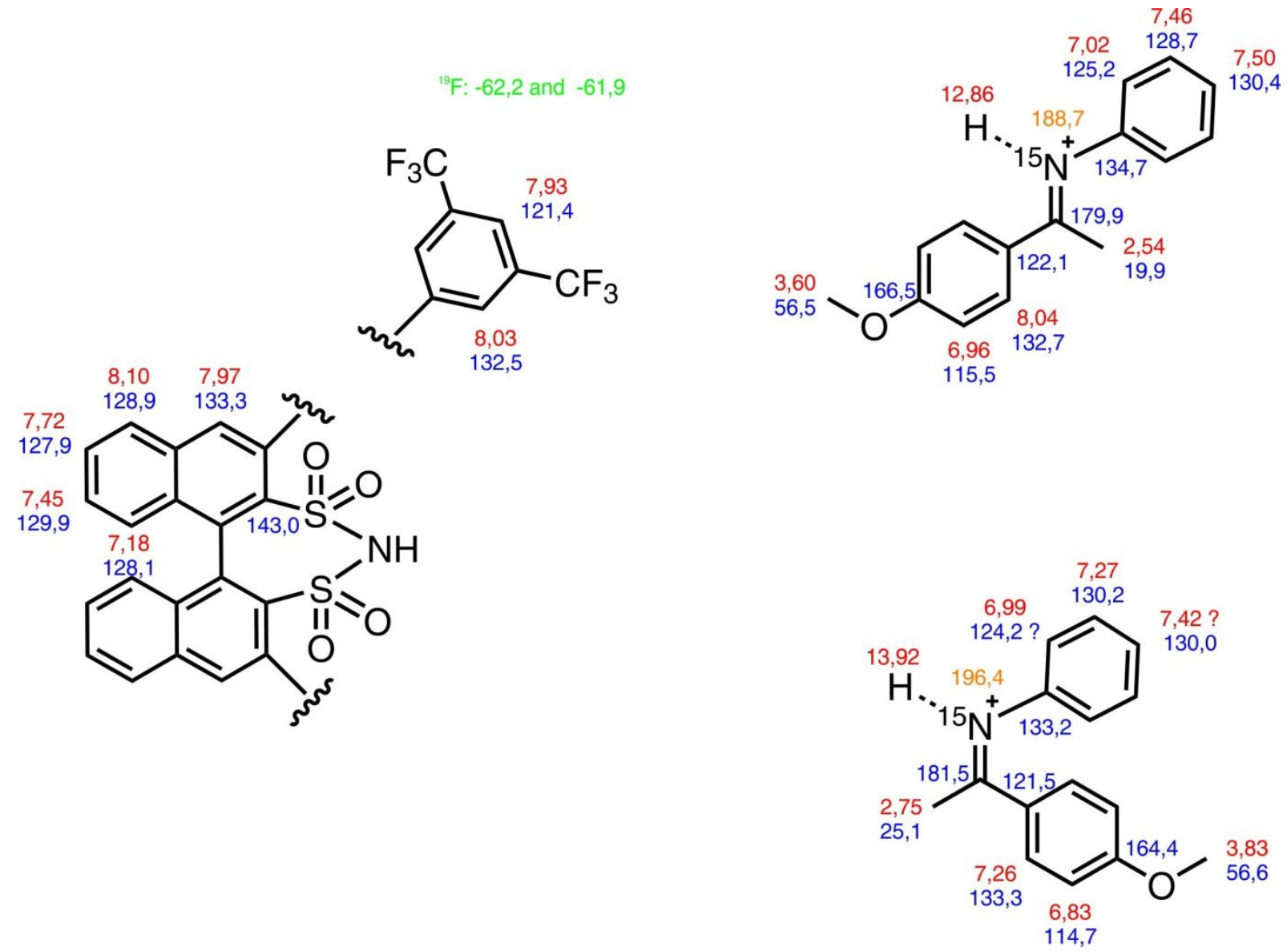


\section{$5.2 C F_{3}-D S I 1 f / 2 a\left(C D_{2} C l_{2}, 180 K\right)$}
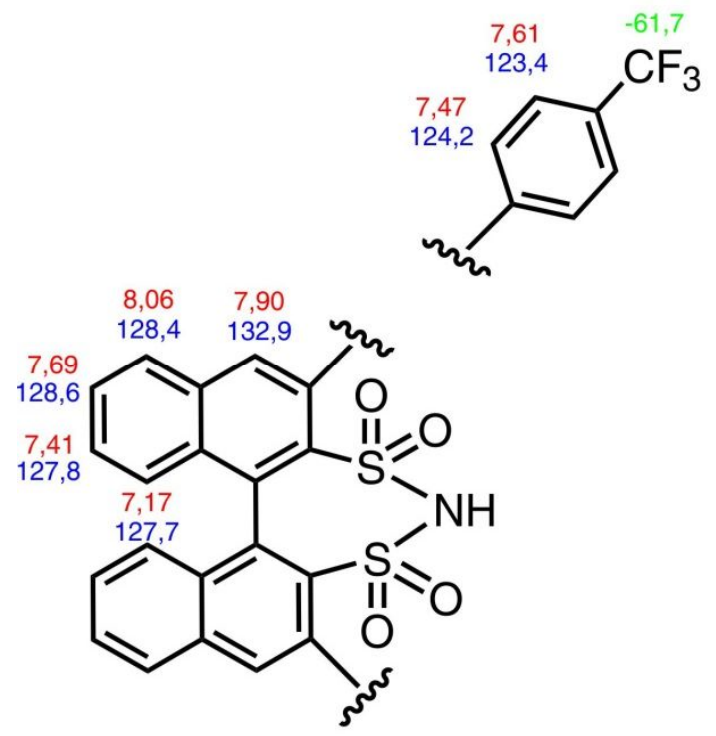

\section{$5.3 C F_{3}-D S I 1 f / 2 b\left(C D_{2} C l_{2}, 180 K\right)$}
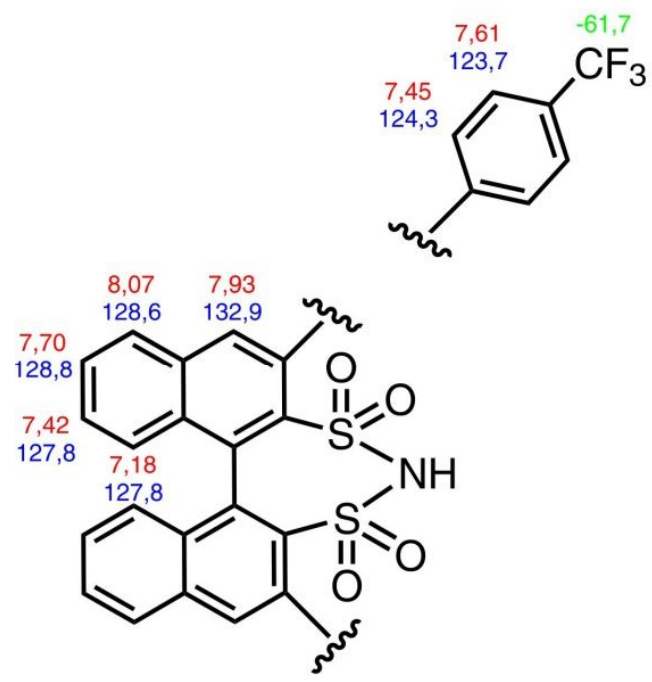
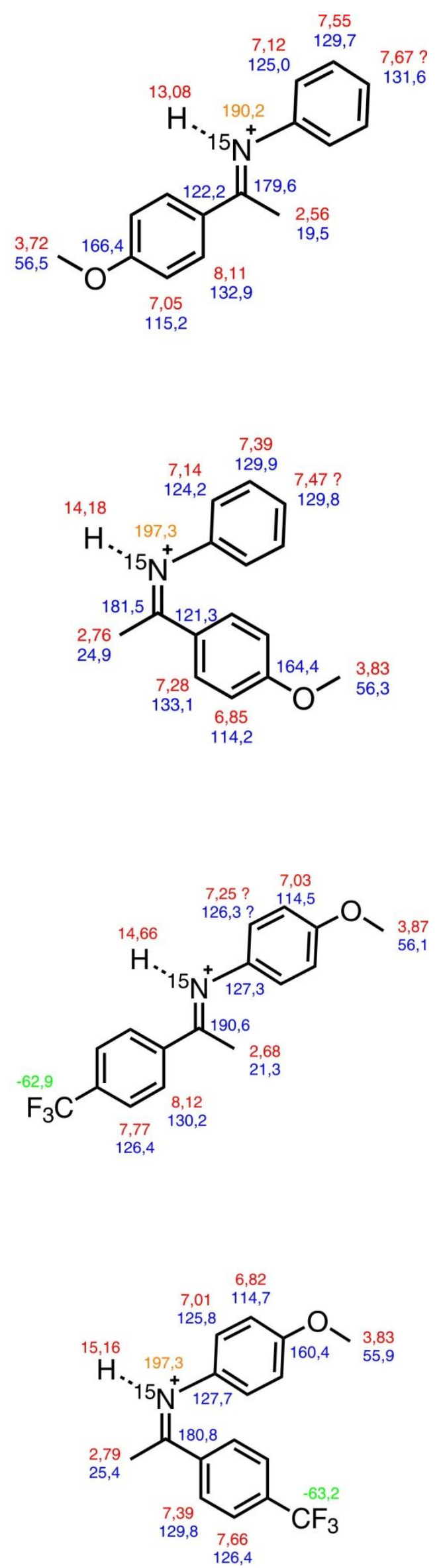


\section{$6 \quad{ }^{1} \mathrm{H}$ Spectrum of a Binary DSI 1e-Complex with an Excess of Imine 2a (1:2)}

a) free imine $2 a$

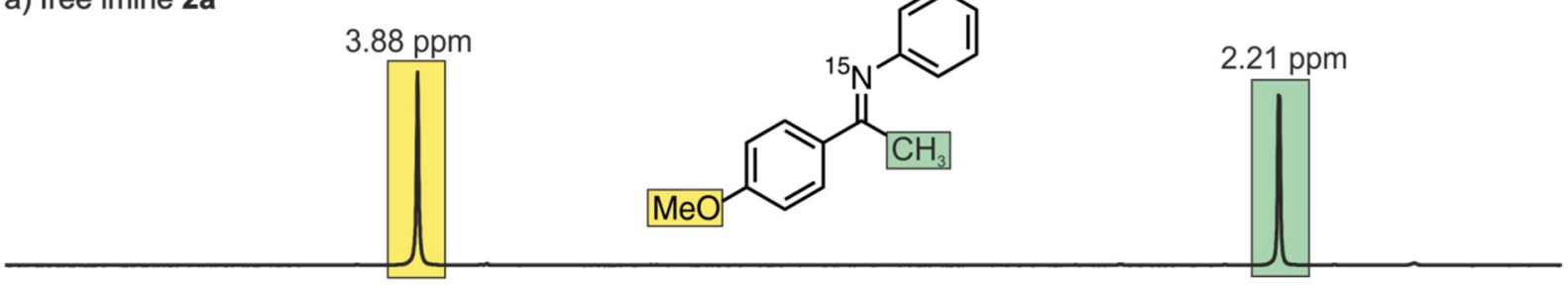

b) $\left(\mathrm{CF}_{3}\right)_{2}$-DSI 1e : imine $(1: 1)$

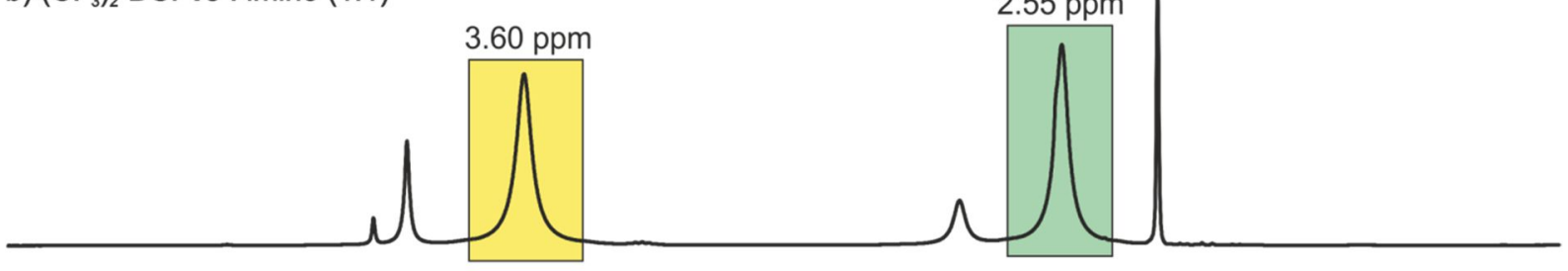

c) $\left(\mathrm{CF}_{3}\right)_{2}$-DSI 1e : imine $2 \mathrm{a}(1: 2)$

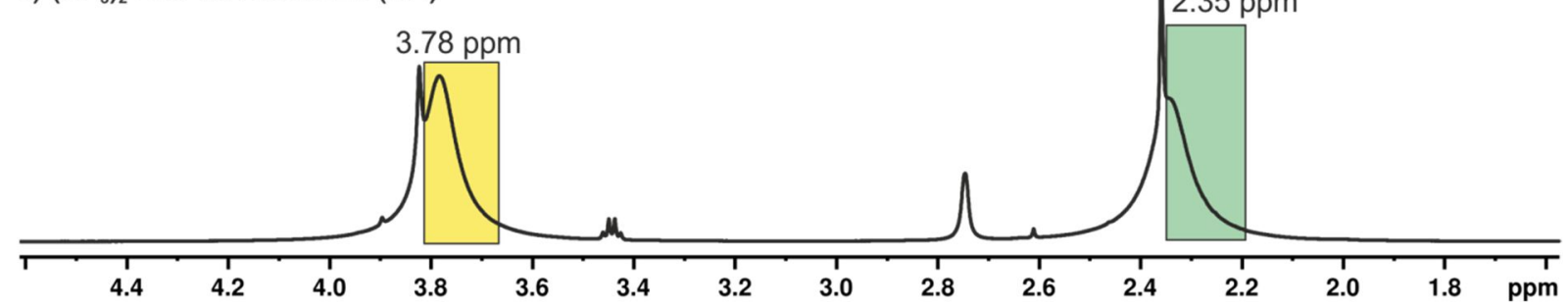

Figure S 17: When comparing the aliphatic region of the ${ }^{1} \mathrm{H}$-spectra from a) the free imine $\left.2 \mathrm{a}, \mathrm{b}\right)$ the $1: 1$ sample of DSI 1e and imine $\mathbf{2 a}$ and c) the 1:2 sample of DSI 1e and imine 2a it became obvious that the free $E$-imine is even at $180 \mathrm{~K}$ in fast exchange with the $E$-imine in the binary complex. All samples were prepared in $\mathrm{CD}_{2} \mathrm{Cl}_{2}$. 


\section{Predicted Structures of the DSI-Complexes by Calculations}

All geometries were optimized at TPSS/def2-SVP ${ }^{[8]}$ level of theory using empirical dispersion correction $\mathrm{D}^{[9]}$ in continuum of DCM with SMD model. ${ }^{[10]}$ Software used for the geometry optimization was Gaussian 09.D01.[5]

\subsection{E-complexes}

a)

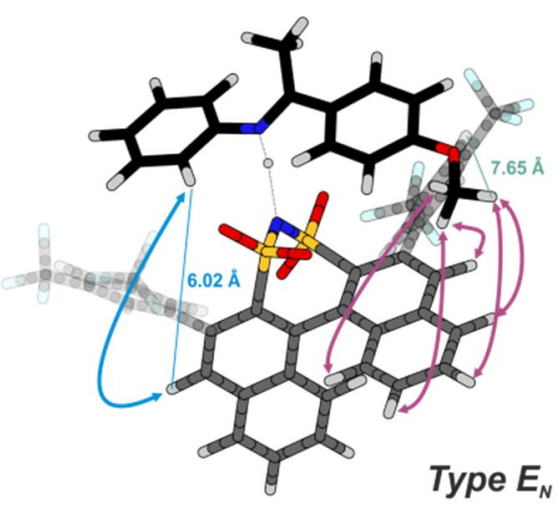

b)

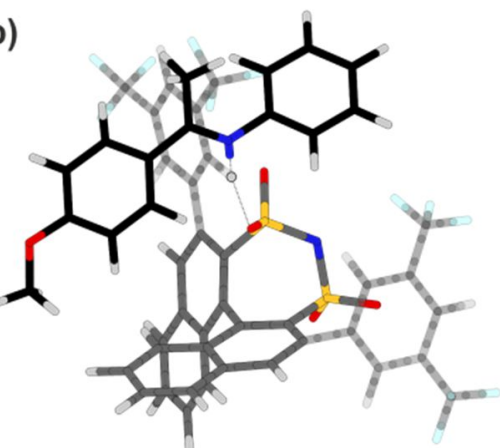

Type I $E_{0}$

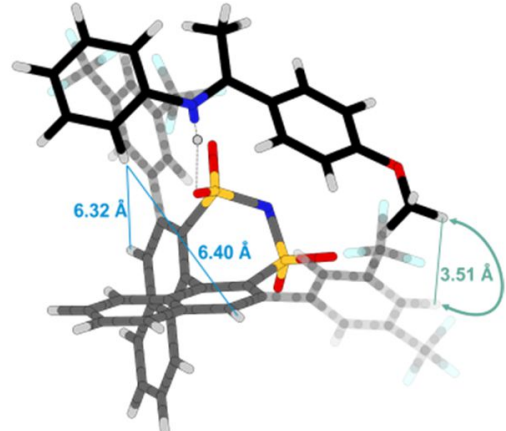

Type II $E_{0}$

Figure S 18: Calculations predict for the DSI/imine 2a-complexes the existence of a) one stable structure, where a hydrogen bond to the nitrogen of the DSI-catalyst (type $E_{N}$ ), is formed and b) several orientations with a hydrogen bond to one of the oxygens. The two most stable structures are shown. While the purple and blue NOEs are not unambiguous for one structure, type // $E_{o}$ of both DSI/2a-complexes could be identified by the observation of the green NOE. All distances given in this figure were obtained from calculations.

\section{Type I $E_{N}$}

1\1|GINC-LOGINIFOptIRTPSSTPSSIdef2SVPIC51H32F12N2O5S2\JHIOEI30-Aug-201

8101॥p tpsstpss/def2svp empiricaldispersion=gd3 int=ultrafine opt scr

$\mathrm{f}=($ smd, solvent $=$ ch2cl2, read) $)$ titlell $0,1 \backslash \mathrm{C}, 0.8793045694,2.4370740122,-0$

$.8997212825 \backslash \mathrm{C}, 1.9302273186,1.6724719242,-0.3844562484 \backslash \mathrm{C}, 3.245319521,1$.

$7001067891,-0.9492357743 \backslash \mathrm{C}, 3.4570376202,2.4979815347,-2.0685391948 \backslash \mathrm{H}, 4$

$.4599508164,2.5475434532,-2.5059157872 \mathrm{lC}, 2.4193679979,3.2760222289,-2$.

6492005675 IC , 1.1066059342,3.2573047958,-2.0577656131।C,0.0709701558,4.

$0257923496,-2.6690513408 \mathrm{H},-0.9369375409,4.0023370859,-2.2456273811 \mathrm{lC}$,

$0.3278145287,4.7885735028,-3.7976449131 \mathrm{lH},-0.4798380744,5.3690396033,-$

4.2562525762।C,1.6288446013,4.8212468968,-4.3687515158ไH,1.8161315095,

$5.4313180825,-5.2588981347 \backslash \mathrm{C}, 2.6531537478,4.0775039373,-3.8063770881 \mathrm{lH}$

,3.6579809815,4.0881316418,-4.2425208166\C,-0.5024257133,2.4373748589, $-0.3017557045 \backslash \mathrm{C},-1.4038882974,1.3917203254,-0.5198865283 \backslash \mathrm{C},-2.77190564$ $32,1.4652585884,-0.0958119282 \backslash C,-3.160957321,2.5681615165,0.6580714868$ $\mathrm{IH},-4.2047977763,2.656027745,0.9777348775 \backslash \mathrm{C},-2.2580962191,3.6155688245$ 
,0.9871080351।C,-0.9130992171,3.5727882206,0.4768516091 IC,-0.021115543 $2,4.6372275077,0.8008851195 \mathrm{lH}, 1.0083853212,4.6045278626,0.4320720322 \mathrm{IC}$ $,-0.442794021,5.6965763129,1.5898574859 \backslash \mathrm{H}, 0.2559461001,6.5029541244,1$. $8368190147 \backslash \mathrm{C},-1.7737374077,5.7431494266,2.0865002864 \backslash \mathrm{H},-2.0915813408,6$ $.5878670533,2.7070097875 \backslash \mathrm{C},-2.6635943887,4.7230302045,1.7907327944 \mathrm{IH},-$ $3.6905320855,4.7483933439,2.1714000432 \backslash \mathrm{C},-3.8101698451,0.4866122534,-0$ $.5231034199 \backslash \mathrm{C},-4.7440057987,-0.025534019,0.3969375117 \backslash \mathrm{C},-3.9142226523$, $0.110919795,-1.8767009446 \backslash C,-5.7422204749,-0.9139618863,-0.0295509129 \mid$ C,-4.9225939292,-0.7675533207,-2.2940298661।C,-5.8414899552,-1.2937369 $237,-1.3759200189|\mathrm{C}, 4.4061508207,0.9644768458,-0.3707728548| C, 5.161171$ $3435,0.092318704,-1.175342499 \backslash C, 4.8009010034,1.1781287429,0.9631828782$ IC,6.2857386341,-0.5603901997,-0.6474621528|C,5.9281870223,0.525029739 $8,1.4788948822 \mathrm{IC}, 6.6791502523,-0.3508770469,0.6817649692 \mathrm{H}, 4.861304007$ $8,-0.086765208,-2.2116264302 \mathrm{lH},-3.2029662156,0.5072381624,-2.607244179$ $5 \backslash \mathrm{H}, 4.2270583076,1.8598059825,1.5970464892 \backslash \mathrm{H}, 7.5592725014,-0.856381354$ $3,1.0873317254 \backslash \mathrm{H},-4.6794701766,0.2519999363,1.4518347583 \backslash \mathrm{H},-6.62119604$ $37,-1.9862230662,-1.7030964481 \backslash C, 6.2956289687,0.7312105313,2.927405130$ $5 \backslash \mathrm{C}, 7.0568599282,-1.5381962903,-1.4986025547 \backslash \mathrm{C},-6.6873066023,-1.529122$ $9953,0.9715481524 \backslash C,-5.0239198391,-1.1155393415,-3.7584421491 \backslash F, 6.9196$ $366522,-1.292172789,-2.8236392371 \mathrm{~F}, 6.6415539418,-2.8243745276,-1.2980$ 219178IF,8.384349733,-1.5225193993,-1.2192315046IF,7.5933494324,0.4302 40922,3.1808542591 IF,5.5453255968,-0.0497418387,3.7523499374IF,6.09580 $70569,2.0136903507,3.3263082573 \mathrm{IF},-5.5863283766,-0.1078329339,-4.48175$ 42023।F,-5.7783968287,-2.2201702647,-3.978065067\F,-3.8042193801,-1.34 $57747516,-4.3087621861 \mathrm{IF},-6.2870616964,-2.7796152423,1.3450306849 \mathrm{IF},-7$ $.9420097922,-1.6671749119,0.4726536276 \mathrm{VF},-6.7864870819,-0.7991547147,2$ $.1102851556 \mathrm{IN}, 0.4420689618,-0.5699189591,0.0152655714 \backslash \mathrm{S}, 1.4155565418,0$ $.4526830603,0.857533517 \backslash 0,2.5323025141,-0.4102662534,1.3293531381 \backslash 0,0$. $7098250976,1.2219516397,1.919078446$ IS $,-0.6546374438,-0.1498365718,-1.1$ 461299688।O,-1.6215952966,-1.2764054651,-1.1363088403।O,-0.0197844346, $0.1849580941,-2.4471726293 \backslash \mathrm{H}, 0.190906313,-2.1909950334,0.5446898822 \backslash \mathrm{C}$, $-3.0484947266,-0.6842903694,3.579890546 \mid C,-3.6929287735,-1.8922952042$, $3.217659322 \backslash \mathrm{H},-4.7542111696,-2.0090710279,3.4494066567 \backslash \mathrm{C},-2.9948092084$ $,-2.8824267368,2.5382306682 \backslash \mathrm{H},-3.5287483654,-3.7837230099,2.2226818097$ IC,-1.6289295692,-2.6971698197,2.1907977269lC,-0.9845750513,-1.5122853 $525,2.6243251569 \mathrm{H}, 0.0861072307,-1.3787748869,2.4572121853 \backslash \mathrm{C},-1.673442$ $4367,-0.5139367722,3.3076816978 \backslash \mathrm{H},-1.1360180501,0.3910911976,3.5961713$ 582\C,-0.9235610285,-3.6852429867,1.3768083057\C,-1.3054233091,-5.1323 $080561,1.4352118742 \backslash \mathrm{H},-0.4256724228,-5.7780759527,1.2805063834 \backslash \mathrm{H},-2.03$ $98401674,-5.3669016202,0.6426386868 \mathrm{lH},-1.7628145888,-5.3670425422,2.40$ $70566827 \backslash \mathrm{N}, 0.0522356867,-3.2452666404,0.6029891421 \backslash \mathrm{C}, 0.9501959339,-3.9$ $20768938,-0.2709837416 \backslash \mathrm{C}, 2.2368826465,-3.3499814082,-0.3849736417 \backslash \mathrm{H}, 2$. $4850591288,-2.4573381286,0.1994943728 \mid C, 3.1759248568,-3.9279295611,-1$. $2457970213 \mathrm{lH}, 4.1769557829,-3.4921571939,-1.3206397548 \mathrm{IC}, 2.8364597559,-$ $5.0584278012,-2.0077236152 \backslash \mathrm{C}, 1.5451022184,-5.6000458254,-1.9151688705 \backslash$ $\mathrm{H}, 1.2655191786,-6.4635559799,-2.5278767051 \mathrm{lC}, 0.5931339285,-5.035764259$ $9,-1.0542876031 \backslash \mathrm{H},-0.4213454125,-5.4372410713,-1.0309341718 \mathrm{lH}, 3.572551$ $0502,-5.5071268663,-2.6832058097 \backslash 0,-3.8297056052,0.2482834473,4.167126$ 7187\C,-3.2551405843,1.5220837739,4.5080560267\H,-4.0790319312,2.11315 $11006,4.9338780429 \mathrm{lH},-2.4545996251,1.4074291614,5.2607108212 \mathrm{lH},-2.8590$ $602641,2.0290787454,3.610537533$ IIVersion=ES64L-G09RevD.01।State=1-AlHF $=-4440.6092707 \backslash \mathrm{RMSD}=4.913 \mathrm{e}-09 \mathrm{RMSF}=1.978 \mathrm{e}-06 \mathrm{IDipole}=-1.5625845,0.33426$ 63,1.2714596lQuadrupole=-29.7197826,45.1747037,-15.4549212,-0.4600814, -29.5693002,-4.5919497/PG=C01 [X(C51H32F12N2O5S2)]॥@ 


\section{Type I Eo}

1 \1।GINC-WORKER1IFOptIRTPSSTPSSIdef2SVPIC51H32F12N2O5S2\JHIOEI30-Apr-2 01910।॥p tpsstpss/def2svp empiricaldispersion=gd3 int=ultrafine opt $s$ $\mathrm{crf}=(\mathrm{smd}$, solvent $=\mathrm{ch} 2 \mathrm{cl} 2$, read) $\|$ title $\| 0,1 \backslash \mathrm{C},-2.2341655662,3.4431898563$ ,2.5465188579IC,-3.1707940399,2.4871149451,2.1402348516IC,-4.542576601 $7,2.5568732356,2.5518338457 \backslash \mathrm{C},-4.9177712073,3.5894973786,3.4078378573 \backslash$ $\mathrm{H},-5.96647431,3.67335657,3.712709673 \backslash \mathrm{C},-3.9950287375,4.5572350017,3.88$ 38068921।C,-2.6259568174,4.4933822182,3.4471480238\C,-1.7009934668,5.4 $507820517,3.963564172 \mathrm{lH},-0.6506999477,5.4013656054,3.6635097889 \mathrm{IC},-2.1$ $178163176,6.4322693093,4.849514834 \backslash \mathrm{H},-1.3928359946,7.1547336402,5.2395$ 634824IC,-3.4766266244,6.5083154926,5.2595156567\H,-3.7907598478,7.292 $8354318,5.9562702621 \mathrm{IC},-4.3966226722,5.5875479721,4.7862315324 \mathrm{IH},-5.44$ $45275333,5.629578955,5.1028960897 \backslash \mathrm{C},-0.7932582005,3.4113150843,2.11868$ 7624IC,0.1160615605,2.5042619206,2.6694249247\C,1.5191148478,2.5634418 $233,2.3843430295 \backslash \mathrm{C}, 1.9577683334,3.5053245155,1.4597969397 \backslash \mathrm{H}, 3.02815662$ $17,3.5793607187,1.2391384118 \backslash \mathrm{C}, 1.0638856816,4.4056628664,0.8173067102 \backslash$ C,-0.3328864365,4.3807573552,1.1645446668IC,-1.2184601818,5.2916799116 $, 0.5160238281 \backslash \mathrm{H},-2.2849738529,5.2652594584,0.7582841888 \backslash \mathrm{C},-0.741401949$ $6,6.1928865552,-0.4232702997 \backslash \mathrm{H},-1.4346192141,6.8817173503,-0.917861238$ 5IC,0.6402003192,6.2268471244,-0.7555662126।H,1.0022325699,6.946010239 $6,-1.4981606155 \backslash \mathrm{C}, 1.5245795271,5.3503359098,-0.1477900259 \backslash \mathrm{H}, 2.59018730$ $84,5.3650666036,-0.4016542719 \mathrm{CC}, 2.5334830264,1.7306094507,3.0946404536$ IC,3.452030336,0.9521121762,2.3714342299|C,2.6281866129,1.7678722478,4 $.5005441185 \backslash \mathrm{C}, 4.4348728498,0.2079349792,3.0455501461 \backslash \mathrm{C}, 3.6145230868,1$. $026029463,5.1622486639 \backslash \mathrm{C}, 4.5233396326,0.234690052,4.4423610059 \backslash \mathrm{C},-5.61$ $80977413,1.6283964979,2.0987616019 \mathrm{IC},-6.4588907761,1.0290393609,3.0522$ 424627\C,-5.8695003688,1.3979480406,0.730587338IC,-7.5299081123,0.2137 $265714,2.6479331362 \backslash \mathrm{C},-6.9329636833,0.5773261302,0.3398420644 \backslash \mathrm{C},-7.775$ $9185076,-0.0214306155,1.291770335 \mathrm{H},-6.2705140627,1.1916599479,4.11769$ $76061 \backslash \mathrm{H}, 1.9325791626,2.3851614056,5.0758391661 \backslash \mathrm{H},-5.2299341049,1.85748$ $71186,-0.0257697082 \backslash \mathrm{H},-8.6016595384,-0.6651438566,0.978538189 \mathrm{H}, 3.3892$ $669039,0.9137571771,1.2796349354 \mathrm{H}, 5.291293785,-0.342425469,4.96219996$ 82IC,-7.1279023163,0.2398584191,-1.1160472493।C,-8.3644632177,-0.46382 $84293,3.7043622413|C, 5.3741935105,-0.6523968551,2.2375743028| C, 3.66883$ $12356,1.0538020666,6.6692223064 \mathrm{IF},-8.6899166306,0.3791728521,4.7173618$ 83IF,-7.6928352093,-1.5064681239,4.2811059681IF,-9.5190975447,-0.97107 $13644,3.2141472447 \backslash F,-8.4404313459,0.1870347572,-1.4610554588 \backslash F,-6.604$ $1782581,-0.9846877358,-1.4114293174$ IF,-6.5304468966,1.1296097531,-1.94 $34615775 \mathrm{IF}, 4.8521036599,0.6067400641,7.1550885412 \mathrm{IF}, 2.7000075737,0.272$ $3661189,7.228707186$ IF $, 3.4800009223,2.3053541177,7.1621364154 \mathrm{IF}, 4.74417$ 26858,-1.7435834098,1.7234486438IF,6.419329729,-1.110909512,2.96839666 $33 \mathrm{IF}, 5.8921249067,0.0231896425,1.1777124528 \mathrm{~N},-1.471526962,0.296412964$ $9,2.343940863 \backslash S,-2.4542271786,1.0496635137,1.2597666542 \backslash O,-3.495719487$ $9,0.0475095541,0.9230313755 \backslash 0,-1.6981177188,1.6229139314,0.1142988105 \backslash$ $\mathrm{S},-0.6321992753,1.0644846351,3.491355285 \backslash \mathrm{O}, 0.4043895024,0.1146083095,3$ $.9632709964 \backslash \mathrm{O},-1.514264742,1.6392008313,4.5801887093 \backslash \mathrm{H},-1.5263066488,0$ $.6324203706,6.0083885951 \backslash \mathrm{C},-3.4514130722,-2.573134037,3.131779854 \mathrm{IC},-2$ $.200597365,-3.1913398867,3.3785877865 \backslash \mathrm{H},-1.863506451,-3.9788749709,2.6$ 981481773।C,-1.4155348207,-2.7849338462,4.4461011156।H,-0.4399560013,$3.2573614834,4.5888406495|C,-1.83185285,-1.7282543765,5.3029386316| C$,3.0916987214,-1.1229521769,5.0432387831\H,-3.4665179783,-0.3364186473, $5.7059734205 \backslash \mathrm{C},-3.9002343961,-1.5427890269,3.9918880385 \mathrm{\backslash H},-4.873144519$ $6,-1.0724155461,3.839429938 \mid C,-0.9805675964,-1.2591677242,6.3877443843$ IC,-0.0106629421,-2.1699030181,7.0705689579\H,-0.4963556706,-2.6630084 $123,7.9325572524 \backslash \mathrm{H}, 0.8736079349,-1.6205946179,7.4283805429 \backslash \mathrm{H}, 0.3063921$ 
$563,-2.9689575499,6.3853290243 \backslash \mathrm{N},-1.0876600449,0.019241021,6.735154005$ IC,- $0.4521262797,0.7518204445,7.7722493406 \backslash C,-0.1003554909,2.086428918$ $3,7.4775610905 \backslash \mathrm{H},-0.3237395185,2.4877284819,6.4844166673 \mathrm{IC}, 0.540949317$ $9,2.864035897,8.4463048763 \backslash \mathrm{H}, 0.8275764329,3.893164534,8.2064242206 \backslash \mathrm{C}, 0$ $.8290306015,2.3244876572,9.7106130305 \mathrm{IC}, 0.4475548656,1.0069700961,10.0$ $084203079 \backslash \mathrm{H}, 0.6464223364,0.5881666786,11.0005639929 \backslash \mathrm{C},-0.1983253374,0$. $2153497856,9.0491914703 \mathrm{lH},-0.521965096,-0.7954547714,9.3057137923 \backslash \mathrm{H}, 1$. $338301409,2.932671705,10.4654471345 \backslash 0,-4.1392592796,-3.0404837899,2.07$ 70030059IC,-5.3758334947,-2.3988699395, 1.7082614467\ل ,-5.6924570836,-2 $.8811806172,0.7724662602 \backslash \mathrm{H},-6.1425489269,-2.5565178585,2.4863848875 \mathrm{lH}$, $-5.2045974214,-1.3258159431,1.5354143275$ IIVersion=ES64L-G09RevD.01।Sta te $=1-A l H F=-4440.6063984 \backslash$ RMSD $=5.657 \mathrm{e}-09 \backslash \mathrm{RMSF}=2.947 \mathrm{e}-06 \backslash \mathrm{Dipole}=0.0053561$ ,1.9117424,4.9997181 \Quadrupole=-20.1980373,19.4357579,0.7622795,1.559 7429,-2.5994384,-18.6795613/PG=C01 [X(C51H32F12N2O5S2)]॥@

\section{Type II Eo}

1 \1।GINC-WORKEROIFOptIRTPSSTPSSIdef2SVPIC51H32F12N2O5S2IJHIOEI30-Apr-2 019101॥p tpsstpss/def2svp empiricaldispersion=gd3 int=ultrafine opt s crf $=($ smd, solvent $=$ ch2cl2, read) $) \mid$ title $\| 0,1 \backslash C, 0.0345938751,1.4654756589$, 1.913257879IC,-0.6173866824,0.4820596832,1.1577558464IC,-2.0100737584, $0.1940072739,1.3399510689 \mathrm{lC},-2.6865842824,0.8306114571,2.3743030869 \mathrm{lH}$, $-3.7570401741,0.6406823255,2.5082474204 \mathrm{IC},-2.0387886039,1.7494971804,3$ $.2449548965 \backslash \mathrm{C},-0.6626340502,2.0904843733,3.0044326668 \backslash \mathrm{C},-0.0171654154$, $3.0000898728,3.8936332073 \mathrm{H}, 1.0400998556,3.2358241337,3.7430928355 \backslash \mathrm{C},-$ $0.7102817376,3.566425394,4.9521120997 \backslash \mathrm{H},-0.1974877133,4.2563003667,5.6$ $307386918 \backslash \mathrm{C},-2.079477248,3.2534488192,5.1693477938 \mathrm{H},-2.6154222787,3.7$ $146781475,6.0058105243 \backslash \mathrm{C},-2.7300698176,2.3600814507,4.3336247437 \backslash \mathrm{H},-3$. $7815162881,2.1020900351,4.5004802842 \backslash C, 1.4239906637,1.9165070145,1.571$ $378818 \backslash \mathrm{C}, 2.5315049776,1.0730548847,1.6843096767 \backslash \mathrm{C}, 3.8315533076,1.45927$ $70558,1.230717141 \backslash \mathrm{C}, 4.0109122498,2.7731695004,0.8090403501 \backslash \mathrm{H}, 5.0021368$ $426,3.0970983767,0.4736343107 \backslash C, 2.9300670506,3.6906935253,0.7202452094$ IC,1.5995140231,3.2480022152,1.0514297237\C,0.5150520593,4.1563646077, $0.8509338596 \mathrm{lH},-0.5071889924,3.8301624385,1.0582124616 \mathrm{lC}, 0.7424456981$, $5.4468457006,0.3979280395 \mathrm{lH},-0.10273538,6.1287645194,0.2597849472 \mathrm{lC}, 2$. $0600805803,5.8904412874,0.1039983212 \backslash \mathrm{H}, 2.2222228545,6.9134412372,-0.25$ $17991498 \mid \mathrm{C}, 3.1307006946,5.0251400948,0.2536645818 \mathrm{lH}, 4.149007854,5.3477$ $211747,0.0109937082 \backslash \mathrm{C}, 4.9734050758,0.5134644886,1.0715077512 \mathrm{IC}, 4.81650$ $66667,-0.6412852909,0.2829176498 \mid C, 6.2401726159,0.8109638728,1.6066478$ 697\C,5.9141397967,-1.4779128935,0.0348893713\C,7.3265076182,-0.041246 $8353,1.3636031551 \backslash \mathrm{C}, 7.1746260093,-1.1920355304,0.5752515885 \backslash \mathrm{C},-2.81127$ $49045,-0.6378843442,0.3922944083 \backslash C,-3.5373886067,-1.7513761297,0.84138$ $60357 \backslash C,-2.9358334736,-0.2337756603,-0.9530112862 \backslash C,-4.3631598009,-2.4$ $613062656,-0.0476802618|C,-3.7776160057,-0.9345799297,-1.8232717783| C$, $-4.4935812184,-2.0604525553,-1.3816188069 \mathrm{H},-3.452264883,-2.0702410338$ $, 1.8844279244 \mathrm{|H}, 6.3733932573,1.702612464,2.2250910391 \mathrm{lH},-2.3873965494$, $0.6428014974,-1.3085887204 \backslash \mathrm{H},-5.1531360147,-2.6034191945,-2.0631482491$ $\mathrm{IH}, 3.8327373538,-0.8818085685,-0.1327087463 \backslash \mathrm{H}, 8.0232214203,-1.85441823$ $53,0.3860916105 \backslash C,-3.9289441942,-0.4912421623,-3.2561215901 \backslash \mathrm{C},-5.09467$ $26138,-3.6821343551,0.454082878 \mid C, 5.707392031,-2.6995375635,-0.8242213$ 137\C,8.6910334211,0.277892281,1.9223330119|F,-5.6444203484,-3.4721753 $234,1.678881349 \mathrm{IF},-4.2648730278,-4.7519452763,0.5843825323 \mathrm{IF},-6.097481$ $1094,-4.0634370076,-0.3746889702 \mathrm{IF},-5.2116022553,-0.5839367883,-3.6860$ 09094IF,-3.188351055,-1.26088121,-4.1136211223IF,-3.5303490911,0.78815 $58461,-3.450121778 \mid \mathrm{F}, 9.5514120697,0.6887184326,0.9493517231 \mathrm{IF}, 9.265584$ $6268,-0.8072622803,2.5045380692 \mathrm{IF}, 8.6570585012,1.2604972544,2.85436346$ 24IF,4.7681097957,-3.5329992797,-0.3021095403IF,6.8376717885,-3.425850 
1824,-0.987860924IF,5.2675498451,-2.3675044461,-2.0725702993IN,1.41193 $75361,-1.4021603083,1.1955705528 \mid S, 0.4181060039,-0.650254835,0.1588228$ $335 \backslash O,-0.4326625732,-1.6941408417,-0.4601615304 \backslash 0,1.1781847522,0.20219$ $85517,-0.839579349 \mathrm{IS}, 2.1863682769,-0.5734557126,2.397744146910,3.44243$ $07713,-1.3050864619,2.6804529571 \mid \mathrm{O}, 1.3017859024,-0.3251139469,3.567325$ $9212 \mathrm{lH}, 0.6529361812,-0.1945172308,-2.473225312 \mathrm{IC},-1.2267719221,4.13360$ 96846,-3.8561143385।C,-0.561773401,3.44949795,-2.8098699156।H,-0.32871 $02842,3.9490388906,-1.8665153143 \mid C,-0.1954913446,2.1177315968,-2.97834$ 4305\H, $0.3066713198,1.6257890392,-2.1394248014 \backslash \mathrm{C},-0.460068254,1.417682$ $7057,-4.1864284951 \backslash \mathrm{C},-1.1270055367,2.1274892588,-5.2253868477 \backslash \mathrm{H},-1.368$ $0853701,1.6322411933,-6.1689452985 \backslash C,-1.5070032882,3.4521156908,-5.065$ $1969572 \backslash \mathrm{H},-2.0263388411,3.9902368253,-5.8636189279 \backslash \mathrm{C},-0.1142231209,0.0$ $113160657,-4.3708133184 \backslash \mathrm{C},-0.342365173,-0.6553048864,-5.6959605294 \backslash \mathrm{H}, 0$ $.1751943338,-1.6224080631,-5.7578417219 \backslash \mathrm{H}, 0.0228267661,-0.002152682,-6$ $.5045736523 \backslash \mathrm{H},-1.4211630484,-0.8208991566,-5.8576701134 \mathrm{IN}, 0.4155891374$ $,-0.6783898654,-3.3725764314 \mid C, 0.7083201475,-2.0780847532,-3.312453771$ $8 \mathrm{lC}, 1.9673660703,-2.4653375588,-2.8219090188 \mathrm{lH}, 2.7099331662,-1.7056506$ $331,-2.5634149468 \backslash \mathrm{C}, 2.2552633901,-3.8280286421,-2.6742698132 \backslash \mathrm{H}, 3.23382$ $56265,-4.1321535692,-2.2934709041 \backslash \mathrm{C}, 1.2907073714,-4.7948281814,-3.0002$ $567089 \mathrm{IC}, 0.0293356292,-4.3964944216,-3.4704262021 \backslash \mathrm{H},-0.7344019635,-5.1$ $456883378,-3.7042947114 \backslash \mathrm{C},-0.2720630646,-3.0371113915,-3.6215904465 \mathrm{IH}$, $-1.2681271036,-2.718678649,-3.9369612729 \mathrm{H}, 1.5184832232,-5.8586757991$, $-2.8750092327 \backslash 0,-1.6264699853,5.4162588697,-3.7911456126 \backslash \mathrm{C},-1.35929972$ $46,6.1734160407,-2.596691629 \mathrm{H},-1.7693471247,7.1759851043,-2.784926135$ $4 \backslash \mathrm{H},-1.8644166268,5.7223091225,-1.7245742953 \backslash \mathrm{H},-0.2728843602,6.2429172$ 884,-2.4125426432IIVersion=ES64L-G09RevD.01।State $=1-A l H F=-4440.6024952$ IRMSD=2.644e-09|RMSF=2.938e-06IDipole=-3.2317603,6.0127334,-5.5215452 Quadrupole $=-30.7853429,23.2211136,7.5642293,-4.7518935,-9.8284866,17.8$ 627781 $\mid \mathrm{PG}=\mathrm{C} 01$ [X(C51H32F12N2O5S2)]1@@

\subsection{Z-complexes}

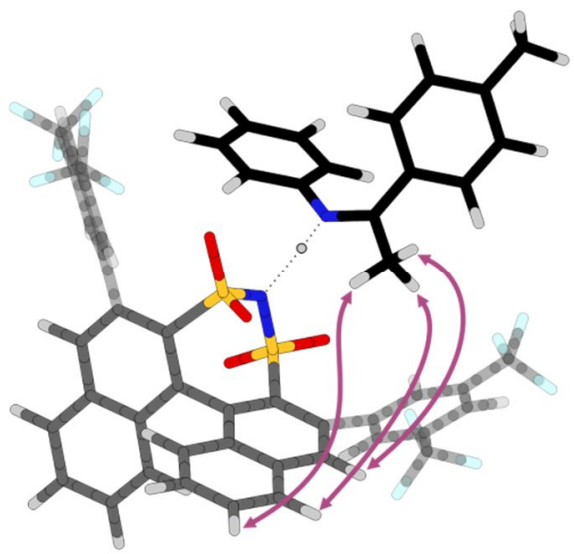

Type $I Z_{N}$

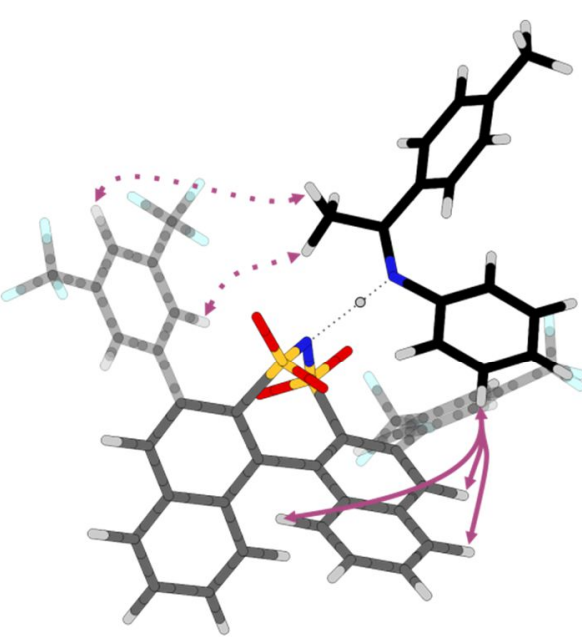

Type II $Z_{N}$

Figure S 19: Calculations predict two stable structures for the DSI/Z-2a-complex, where the hydrogen bond to the nitrogen is formed. Within the $\mathrm{CF}_{3}$-DSI/Z-2b-complex some characteristic NOEs (shown in purple) indicate the existence of both structures (see below). However, for the other complexes it was not possible to identify the different structures. This may be due to the fast exchange of several hydrogen bonded species. 


\section{Type I $Z_{N}$}

1\1|GINC-LOGINIFOptIRTPSSTPSSIdef2SVPIC51H32F12N2O4S2IJHIOEI10-Oct-201 8101॥p tpsstpss/def2svp empiricaldispersion=gd3 int=ultrafine opt scr $\mathrm{f}=($ smd, solvent $=$ ch2cl2, read) $) \mid$ titlell0,1 $\mid C,-0.0062106269,-3.181524239,0$ $.5849268747 \backslash \mathrm{C}, 1.1193012089,-2.4592295897,0.1759338914 \mid \mathrm{C}, 2.4091644458,-$ $2.6705283545,0.7592378934 \backslash \mathrm{C}, 2.5216002348,-3.5938181737,1.79209333 \backslash \mathrm{H}, 3$. $5029304178,-3.7699988512,2.2459022644 \backslash C, 1.4053370189,-4.3392251604,2.2$ $606894547 \backslash \mathrm{C}, 0.1187467171,-4.1444164528,1.6443570045 \backslash \mathrm{C},-0.9958342464,-4$ $.8858938616,2.1393059536 \backslash \mathrm{H},-1.9831526214,-4.7315920823,1.6947864872 \mathrm{IC}$, $-0.8399687007,-5.787430733,3.1805871833 \mathrm{H},-1.7063621385,-6.346491054,3$ $.549931345 \backslash \mathrm{C}, 0.4337201201,-5.9897654305,3.7778332399 \mathrm{H}, 0.5406060122,-6$ $.7070787999,4.5986265444 \backslash \mathrm{C}, 1.5332700114,-5.2768624102,3.3287744 \backslash \mathrm{H}, 2.51$ $76375767,-5.419784749,3.7878007486 \mid C,-1.3587219889,-2.9767543281,-0.04$ 07374695\C,-2.1422731329,-1.8507419231,0.2338976503।C,-3.4450141109,-1 $.6752239228,-0.3337844992 \backslash C,-3.9125371709,-2.6440198015,-1.2149194438 \mid$ $\mathrm{H},-4.9125877895,-2.5336288235,-1.6480220437 \backslash \mathrm{C},-3.1450162181,-3.7921641$ 643,-1.5510063924\C,-1.850175714,-3.9751781391,-0.9496983942\C,-1.0841 $448044,-5.1234676979,-1.3112443339 \backslash \mathrm{H},-0.0894262578,-5.2624007832,-0.87$ $8494271 \backslash \mathrm{C},-1.5842054278,-6.0539850794,-2.2088194794 \mathrm{IH},-0.9816027861,-6$ $.9283294939,-2.4771897738 \mathrm{lC},-2.8706926738,-5.881396303,-2.7877975645 \mathrm{lH}$ ,-3.2530426289,-6.6267701082,-3.4933931612lC,-3.6344932412,-4.77123572 $68,-2.4664533242 \backslash \mathrm{H},-4.6239520371,-4.6241030325,-2.9131015067 \backslash \mathrm{C},-4.3506$ $195324,-0.5411414529,0.015069125 \mathrm{IC},-4.8366315432,0.3122698712,-0.99181$ 92132\C,-4.7756396871,-0.3471902454,1.3417755728IC,-5.7213859056,1.351 $3698782,-0.6682885335 \backslash \mathrm{C},-5.6739117328,0.6832904696,1.6511625784 \backslash \mathrm{C},-6.1$ $497560795,1.5451040324,0.6532252984 \backslash \mathrm{C}, 3.6389898312,-1.9686376419,0.286$ 607791\C,4.3412066609,-1.1172106897,1.1564958615\C,4.1196426107,-2.161 $2835591,-1.0206492082 \backslash C, 5.4817512166,-0.4363880592,0.7074188732$ IC,5.28 $10388754,-1.5031649638,-1.4484598229 \mathrm{IC}, 5.9648885311,-0.6252769194,-0.5$ $956874387 \backslash \mathrm{H}, 3.9702675925,-0.9535229244,2.1713632786 \backslash \mathrm{H},-4.4001272631,-1$ $.0017801637,2.1331371617 \backslash \mathrm{H}, 3.5798579435,-2.8205633285,-1.7060941081 \mathrm{\backslash H}$, $6.8560402368,-0.0938146781,-0.9418017752 \mathrm{H},-4.5046332018,0.1776128683$, $-2.0245823512 \mathrm{lH},-6.8415285204,2.3539308401,0.9034578439 \backslash \mathrm{C}, 5.7515300357$ $,-1.6820878926,-2.8708809173|C, 6.1778979365,0.5583253791,1.6019667566|$ C,-6.2008456367,2.3086930623,-1.7307152629IC,-6.1689394587,0.829733571 ,3.0687819612IF,5.5339389256,0.7437147673,2.7789857852IF,6.2649743062, $1.7832138765,1.0041192267 \backslash F, 7.4517638764,0.1908557,1.8956651238 \backslash F, 7.08$ $66426688,-1.4777563982,-3.0005054574 \mathrm{IF}, 5.1414475655,-0.8091519989,-3.7$ 187527105 IF,5.4877952789,-2.9264882522,-3.3433423291 IF,-7.2221323679,0 $.00357072,3.3229806689 \mathrm{IF},-6.5907747766,2.0897509281,3.3397491753 \mathrm{IF},-5$. $2117400555,0.5210082586,3.9793535427 \backslash \mathrm{F},-5.6832698851,3.5592334906,-1.5$ $510763016 \mathrm{IF},-7.5512929318,2.4551055031,-1.7133363854 \mathrm{IF},-5.8552724401,1$ $.9207444787,-2.9808276564 \backslash \mathrm{N},-0.1373082324,-0.0316102476,0.0023639332 \mathrm{SS}$ $, 0.7748703563,-1.0501652861,-0.9301247951 \backslash 0,1.9955213347,-0.2612221878$ $,-1.2318470457 \backslash 0,0.0242471846,-1.5955589086,-2.089035924 \mid S,-1.27670824$ $51,-0.4998196217,1.0979240656 \backslash 0,-0.6939766184,-1.0754090086,2.33822845$ $61 \backslash \mathrm{O},-2.1382033139,0.6978762832,1.2730416356 \mid C, 3.2681926192,6.91967583$ $15,1.0645083033 \backslash \mathrm{C}, 1.8816887412,6.6822774508,0.9171935963 \mathrm{IH}, 1.186106154$ 
$7,7.5288505751,0.9254663092 \backslash \mathrm{C}, 1.3810292277,5.3906117788,0.7621041118 \backslash \mathrm{H}$ ,0.3024432733,5.2391314065,0.6623146094IC,2.2573562747,4.2769811216,0. $7727385125 \mathrm{IC}, 3.6399003933,4.5032871123,0.9635650958 \mathrm{H}, 4.3369106639,3.6$ $607212293,0.9913366452 \backslash \mathrm{C}, 4.1327707083,5.8064333875,1.0864726022 \mathrm{H}, 5.21$ $01877041,5.9623103621,1.2089656754 \backslash C, 1.7548042958,2.8991378424,0.66522$ 93138IC,2.4247693143,1.8028882189,1.4383568855।H,1.775811382,0.9177358 $518,1.5093867608 \backslash \mathrm{H}, 2.7224257413,2.1488577307,2.4397628609 \backslash \mathrm{H}, 3.34044397$ $77,1.5101371951,0.894823132 \mathrm{lN}, 0.7343724936,2.5450647744,-0.081609598 \mathrm{lH}$ $, 0.4060854634,1.5256944034,0.0180504665 \backslash C, 0.0053365797,3.2511345844,-1$ $.0854490748 \mathrm{IC},-1.3794191793,3.007183095,-1.1548820881 \mathrm{lH},-1.8470268245$, $2.3363162376,-0.4260720119 \backslash \mathrm{C},-2.1347504238,3.6329346763,-2.1558525415 \backslash$ $\mathrm{H},-3.2137150955,3.4586567343,-2.2049506209 \mathrm{IC},-1.5118496856,4.477907514$ $7,-3.0877490569 \backslash \mathrm{C},-0.1237428936,4.6901594998,-3.0258293665 \mathrm{IH}, 0.3685208$ $869,5.330515789,-3.7653763721 \backslash \mathrm{C}, 0.6441261632,4.0768342478,-2.029165927$ $9 \mathrm{H}, 1.7266484936,4.2245086502,-1.9897203444 \mathrm{IH},-2.104837711,4.963004375$ 6,-3.8703007826।C,3.7930554622,8.3274447015,1.1985600948\H,3.299601124 $3,8.8535525768,2.0360469455 \mathrm{H}, 4.8819233265,8.3413255731,1.3688044494 \mathrm{IH}$ ,3.5773283606,8.9108641908,0.2839143287IIVersion=ES64L-G09RevD.01IStat $\mathrm{e}=1-\mathrm{AlHF}=-4365.4354595 \mathrm{IRMSD}=4.598 \mathrm{e}-09 \backslash \mathrm{RMSF}=3.547 \mathrm{e}-06 \backslash \mathrm{Dipole}=1.8393518$, $1.643645,0.5433751 \backslash$ Quadrupole $=-24.3037798,54.9327541,-30.6289743,29.77$ 94239,25.600083,-6.9949413|PG=C01 [X(C51H32F12N2O4S2)]॥@

\section{Type II $Z_{N}$}

1 \1।GINC-WORKER1IFOptIRTPSSTPSSIdef2SVPIC51H32F12N2O4S2 IJHIOEI10-Oct-2 01810।॥p tpsstpss/def2svp empiricaldispersion=gd3 int=ultrafine opt s crf $=($ smd, solvent $=$ ch2cl2, read) $) \backslash t i t l e \| 0,1 \backslash C, 0.2447270992,-3.0307115589$ $, 0.8494143459 \mid \mathrm{C}, 1.125317216,-2.1368585662,0.2225140602 \backslash \mathrm{C}, 2.4861140505$, $-1.9923244527,0.6531945154 \backslash \mathrm{C}, 2.8861483006,-2.6631014232,1.8041950364 \mathrm{IH}$ ,3.9259140105,-2.5783615856,2.1376411693IC,1.9958905614,-3.4862132857, $2.5453085875 \backslash C, 0.6619346828,-3.7004784104,2.0510227049 \mid C,-0.2244619313$ $,-4.5227688223,2.8073771479 \mathrm{lH},-1.2512159725,-4.6642219058,2.4587752463$ IC,0.1960314438,-5.1200145089,3.9855363639\H,-0.5002850537,-5.74114157 $82,4.558960079 \mid \mathrm{C}, 1.5215960469,-4.926999557,4.4606716228 \mathrm{H}, 1.8404796983$ $,-5.4100763956,5.3904972713|C, 2.4035109128,-4.1249268687,3.7546385036|$ $\mathrm{H}, 3.4248398677,-3.9630490209,4.1162653572 \mathrm{CC},-1.1063716259,-3.332396497$ $2,0.2633329999 \backslash \mathrm{C},-2.1187096855,-2.3739835505,0.1872075946 \mid C,-3.3333537$ $979,-2.5905909394,-0.5352124199 \mid C,-3.5909880331,-3.8763715962,-0.99597$ $58424 \backslash \mathrm{H},-4.5235983159,-4.0757643571,-1.534851243 \backslash \mathrm{C},-2.6325439801,-4.91$ $97509371,-0.8771926216|C,-1.3395374785,-4.6340404101,-0.3056146584| C,-$ $0.3450402843,-5.6585041674,-0.3294767154 \backslash \mathrm{H}, 0.6540833865,-5.4474716125$, $0.0604850107 \backslash \mathrm{C},-0.6318344487,-6.9147957799,-0.8401334637 \backslash \mathrm{H}, 0.144173011$ $6,-7.6875883146,-0.8465847264 \backslash \mathrm{C},-1.9213447858,-7.2077636262,-1.3601931$ $94 \mathrm{IH},-2.1344961819,-8.2072532009,-1.7540872923 \mathrm{IC},-2.898397128,-6.22646$ $07177,-1.3859010853 \backslash \mathrm{H},-3.887429821,-6.432895634,-1.8092642605 \backslash \mathrm{C},-4.233$ $0347546,-1.4756627667,-0.9472052094 \backslash \mathrm{C},-3.6761369,-0.3962484129,-1.6668$ $659319 \mid C,-5.6153808088,-1.5023551577,-0.714254309 \backslash C,-4.4902588829,0.64$ $64277512,-2.1142827562 \mid \mathrm{C},-6.4268167831,-0.4499301212,-1.1767662099 \mathrm{IC},-$ $5.8754820763,0.6315525732,-1.8722940742 \backslash C, 3.5158937401,-1.253125281,-0$ 
$.1307386468 \mid C, 4.3829272398,-0.3445325743,0.5001519547 \backslash C, 3.6855476066,-$ $1.5169805517,-1.5049762019|\mathrm{C}, 5.3929853471,0.2960993844,-0.2356981081| \mathrm{C}$ ,4.7000920674,-0.8785683266,-2.2273046522/C,5.5627843058,0.0342616341, $-1.6012082857 \backslash \mathrm{H}, 4.2557859485,-0.1212866797,1.5629023137 \backslash \mathrm{H},-6.059915789$ $5,-2.3308037668,-0.1547681785 \backslash \mathrm{H}, 3.0224673562,-2.2263540879,-2.00827403$ $37 \backslash \mathrm{H}, 6.3573013675,0.5261827699,-2.16790759481 \mathrm{H},-2.6009199148,-0.382679$ $3924,-1.8669356303|\mathrm{H},-6.5117394822,1.4492037013,-2.2211638236| \mathrm{C}, 4.8369$ $388777,-1.1330827556,-3.7077438434 \mid C, 6.2778871911,1.315423121,0.437346$ 9901।C,-3.8870653372,1.8324562232,-2.8237625547\C,-7.914692703,-0.5209 193539,-0.938113577\F,6.4684025499,1.0436145803,1.7531696397IF,5.74796 $05205,2.5709932064,0.374141199 \mathrm{IF}, 7.5042574526,1.3900455783,-0.13802520$ 88IF,6.1195108067,-1.0014088387,-4.1330569991IF,4.0952542311,-0.258559 6017,-4.4419813824IF,4.4238087837,-2.3770010543,-4.0562715708IF,-8.522 $2268402,-1.3912564271,-1.7919866286 \mathrm{IF},-8.5261981361,0.6772277934,-1.10$ $14243747 \mathrm{IF},-8.2060368694,-0.9532987328,0.3164026082 \mathrm{VF},-3.9150107535,2$. 9511058614,-2.0439099847/F,-4.5623433012,2.1455537778,-3.9602075826IF, $-2.5918709554,1.6325073173,-3.1708049375 \mathrm{IN},-0.6619607299,-0.0631077107$ $, 0.00301575161 \mathrm{~S}, 0.3898328955,-0.9265417921,-0.9425148576 \mid 0,1.389841290$ $2,0.0962888109,-1.340854443910,-0.2798783469,-1.6835315781,-2.03249608$ 89IS,-1.7591182513,-0.7946161006,1.00717002721O,-1.1542693173,-1.15386 20109,2.3229046586।O,-2.9482669193,0.0846779368,1.0717963177।C, 1.17649 $64227,7.2461398767,1.8951247212 \backslash \mathrm{C}, 0.8640859742,6.1760467214,2.76564194$ $29 \mathrm{lH}, 0.8344027684,6.3517026345,3.8468611476 \mathrm{lC}, 0.5919595026,4.900356252$ $5,2.2744997072 \backslash \mathrm{H}, 0.3445673263,4.093813929,2.9710321334 \backslash \mathrm{C}, 0.6005329006$, 4.6539631945, $0.8788498277 \backslash \mathrm{C}, 0.8735201964,5.7262602385,0.0013493 \mathrm{lH}, 0.88$ $29089994,5.5620507188,-1.0802265182$ lC $, 1.174854712,6.9952070223,0.50840$ $00535 \backslash \mathrm{H}, 1.4100798676,7.808377659,-0.1869705745 \mathrm{IC}, 0.2559967376,3.323701$ $8194,0.3503286342 \mathrm{lC},-0.6382407478,3.205740218,-0.844930526 \mathrm{lH},-0.824279$ $0588,2.1540457963,-1.1026376626 \mathrm{H},-0.16014951,3.7077758237,-1.70594870$ $37 \mathrm{\backslash H},-1.5917499331,3.727902587,-0.6570880925 \backslash \mathrm{N}, 0.6597138469,2.20202126$ $05,0.9033900171 \mathrm{lH}, 0.1799067071,1.3180372542,0.5404805582 \backslash \mathrm{C}, 1.640024657$ $5,1.9577461658,1.9129227808 \mathrm{IC}, 1.4038100844,0.88322444404,2.7904715233 \mathrm{lH}$ $, 0.4889948647,0.2878351066,2.69910036291 \mathrm{C}, 2.3536126237,0.582106421,3.7$ 764896387\H,2.1699460214,-0.2551977427,4.4578253098\C,3.5291102872,1.3 $406973455,3.8842440699 \mathrm{IC}, 3.7682431793,2.391162931,2.9813667655 \mathrm{lH}, 4.702$ $2202508,2.9582931765,3.0330033098 \mid C, 2.8353022562,2.6987575691,1.985474$ $5576 \mathrm{lH}, 3.0417728116,3.4906923483,1.2617960779) \mathrm{H}, 4.2701329935,1.1022090$ $176,4.65434318 \mathrm{IC}, 1.4995890198,8.6116741866,2.4482909385 \mathrm{IH}, 2.4086674213$ ,8.5713765019,3.07706429161H,1.6671796267,9.3471829596,1.6447405656 $\mathrm{lH}$, $0.6810848103,8.9784506314,3.0943392085 \mathrm{IIVersion}=E S 64 L-G 09 R e v D .01 \mid$ State $=1-\mathrm{AlHF}=-4365.4396613 \mathrm{RMSD}=4.458 \mathrm{e}-09 \mathrm{RMSF}=4.746 \mathrm{e}-06 \mathrm{IDipole}=2.2980543,1$ $.7654466,3.0146497 \backslash$ Quadrupole $=-44.050728,63.265117,-19.214389,15.17512$ 64,21.6884146,4.2021546/PG=C01 [X(C51H32F12N2O4S2)]।@ 


\section{Structure Identification of All Binary Complexes}

\subsection{NOE build-up curve}

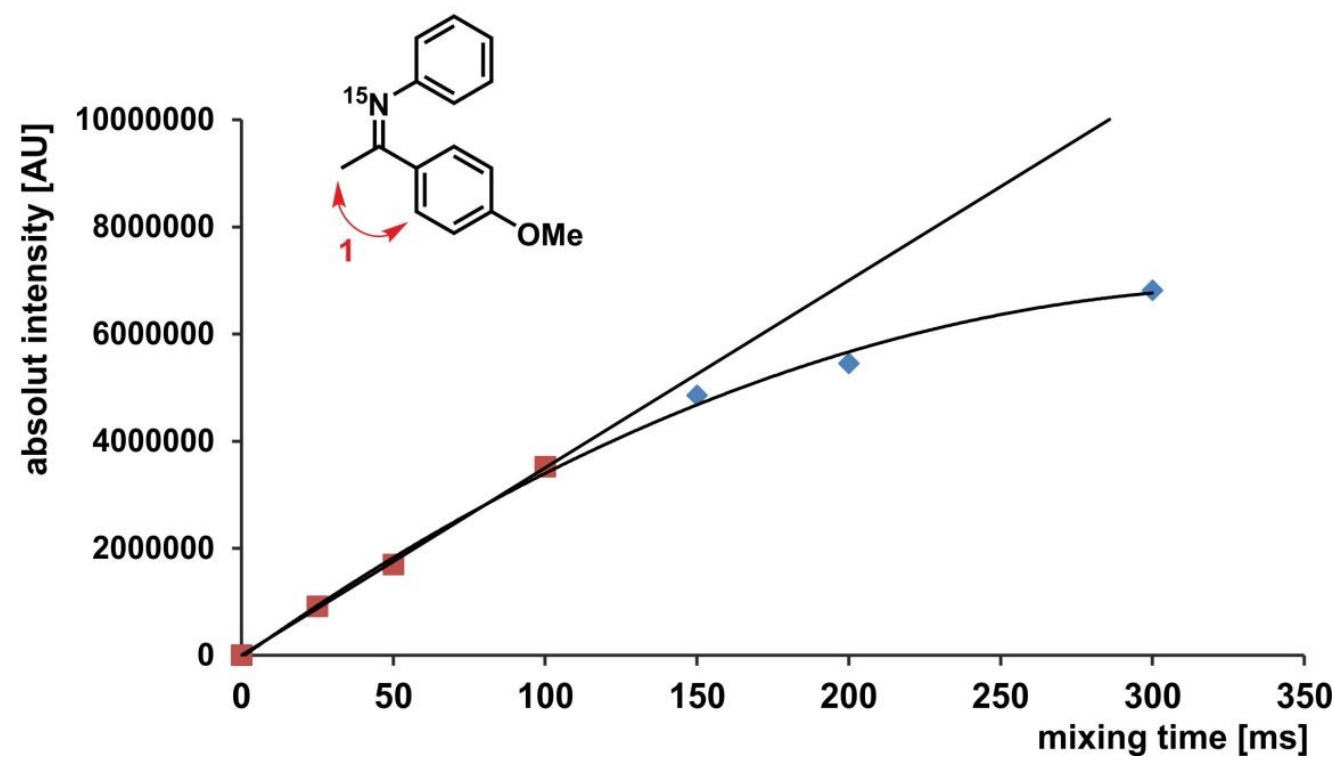

Figure S 20: Selective 1D ${ }^{1} \mathrm{H}-\mathrm{NOESYs}$ of the 1e/2a-complex in $\mathrm{CD}_{2} \mathrm{Cl}_{2}$ at $180 \mathrm{~K}$ with different mixing times were measured. The a-methyl group of the imine was excited. The resulting build-up of the intensity of intramolecular NOE 1 (red arrow) is shown. Due to spin diffusion effects, even with 100 ms mixing time a deviation from a linear build was observed.

\section{$8.2\left(C F_{3}\right)_{2}-D S I$ 1e/2a $\left(C D_{2} C l_{2}, 180 K\right)$}

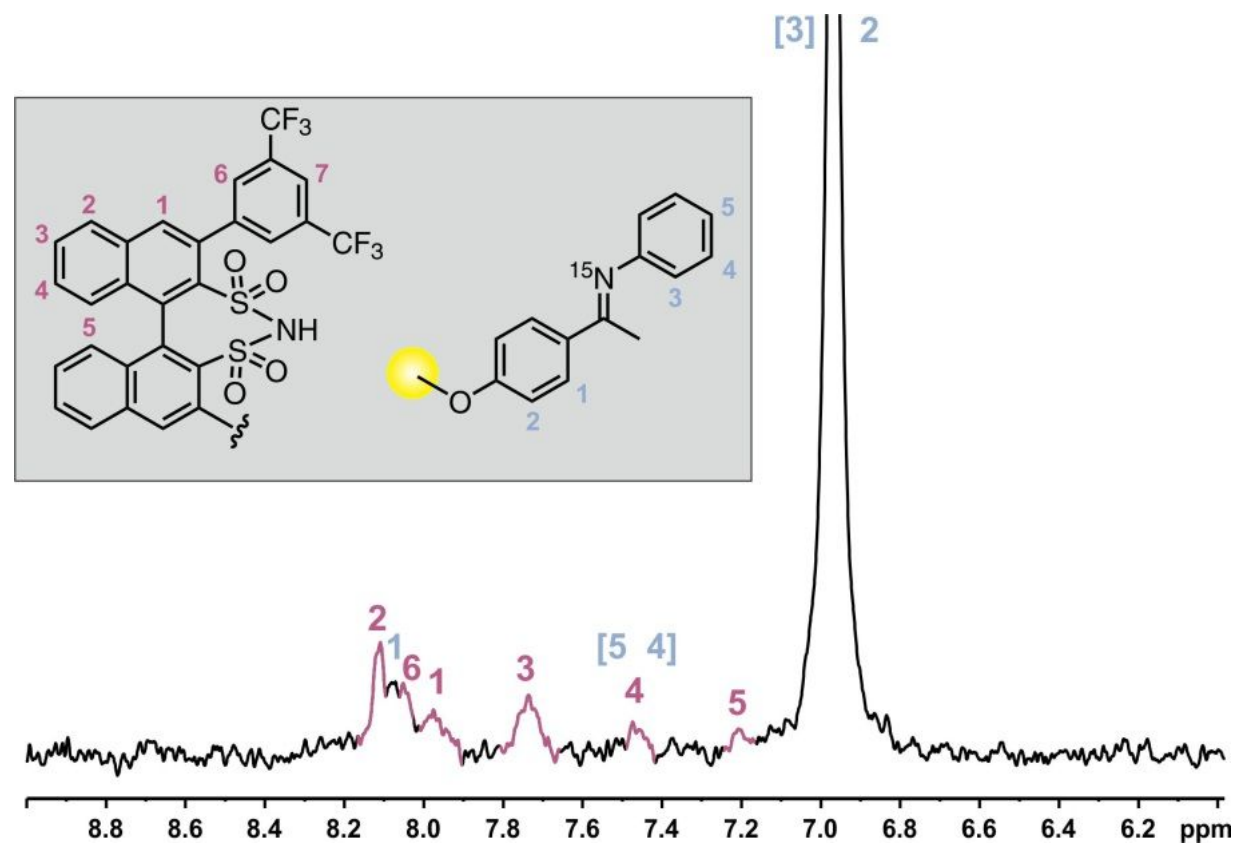

Figure S 21: A section of a selective 1D ${ }^{1} \mathrm{H}-\mathrm{NOESY}\left(\tau_{\mathrm{mix}}=100 \mathrm{~ms}\right.$, RE-Burp pulse $)$ excited on the $p$-methoxy group of $E-2 a$ (yellow circle) at $180 \mathrm{~K}$ in $\mathrm{CD}_{2} \mathrm{Cl}_{2}$ is shown. NOEs are visible to the complete catalyst backbone, indicating the existence of type $E_{N}$. However, also for some of the less populated type Eo-orientations (e.g. type IEO) NOEs between the $p$-methoxy group of $E-2 \mathrm{a}$ (yellow circle) and the BINOL backbone were expected. But due to the fact, that the NOE to proton 2 (purple) of the BINOL backbone is the strongest in combination with the comparison of the calculated distances these groups (type $E_{N}=3.18 \AA$ and type $I E_{O}=5.12 \AA$ ) it can be assumed, that type $E_{N}$ exists in solution. The signals of the protons in brackets are overlapping. 

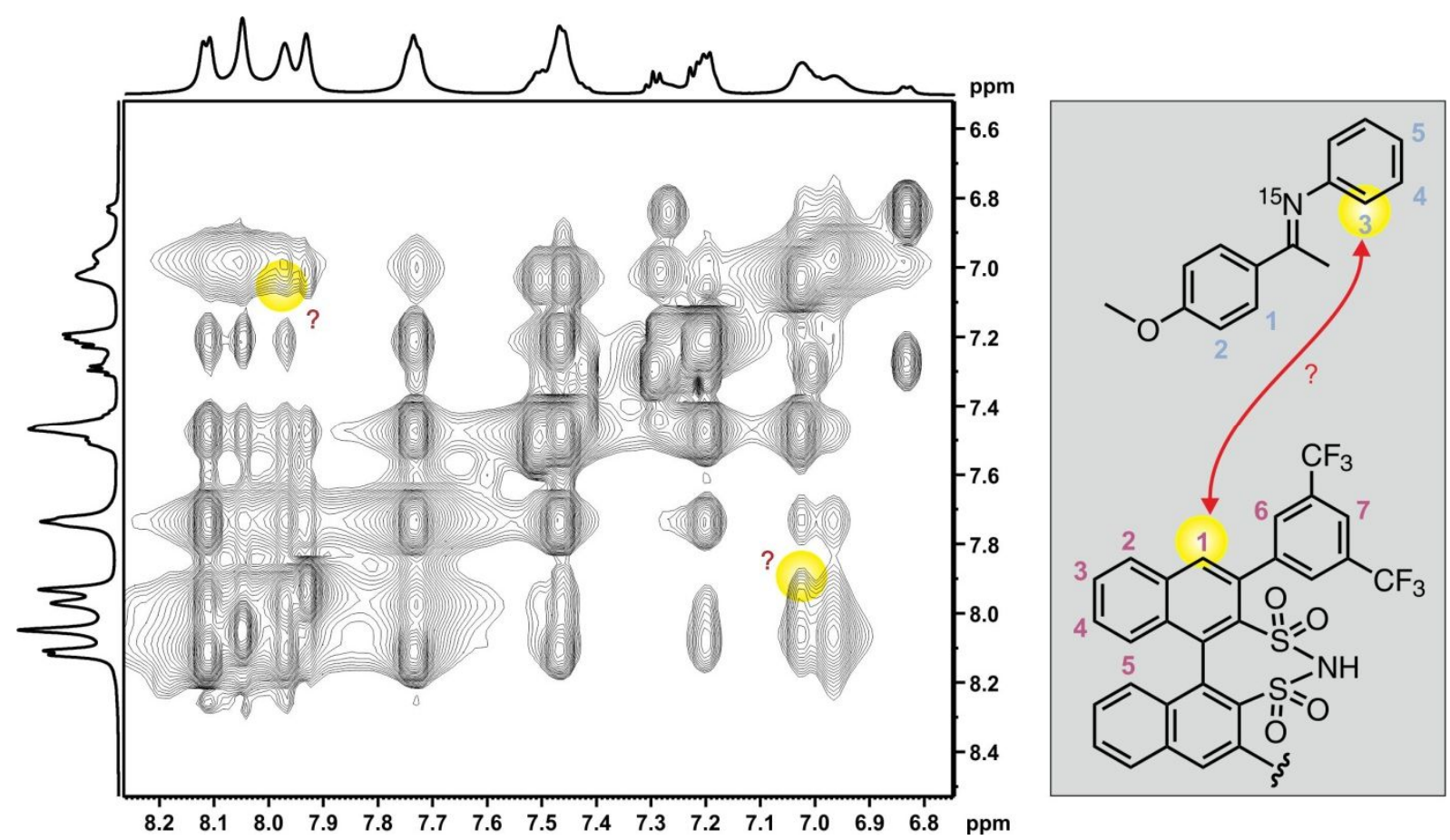

Figure S 22: A 2D ${ }^{1} \mathrm{H},{ }^{1} \mathrm{H}$ NOESY spectrum $\left(\tau_{\text {mix }}=300 \mathrm{~ms}\right)$ of complex $1 \mathrm{e} / 2 \mathrm{a}$ at $180 \mathrm{~K}$ in $\mathrm{CD}_{2} \mathrm{Cl}_{2}$. In contrast to the CPAcomplexes the NOE (labeled in yellow) between proton 1 of the DSI (purple) and proton 3 of the imine (blue) does not identify type IIE $E_{O}$ unambiguously, because these calculated distances for type $E_{N}(6.02 \AA)$ and type IIEO (6.32 $\AA$ and 6.40 $\AA$ ) are similar. See Figure S 18.
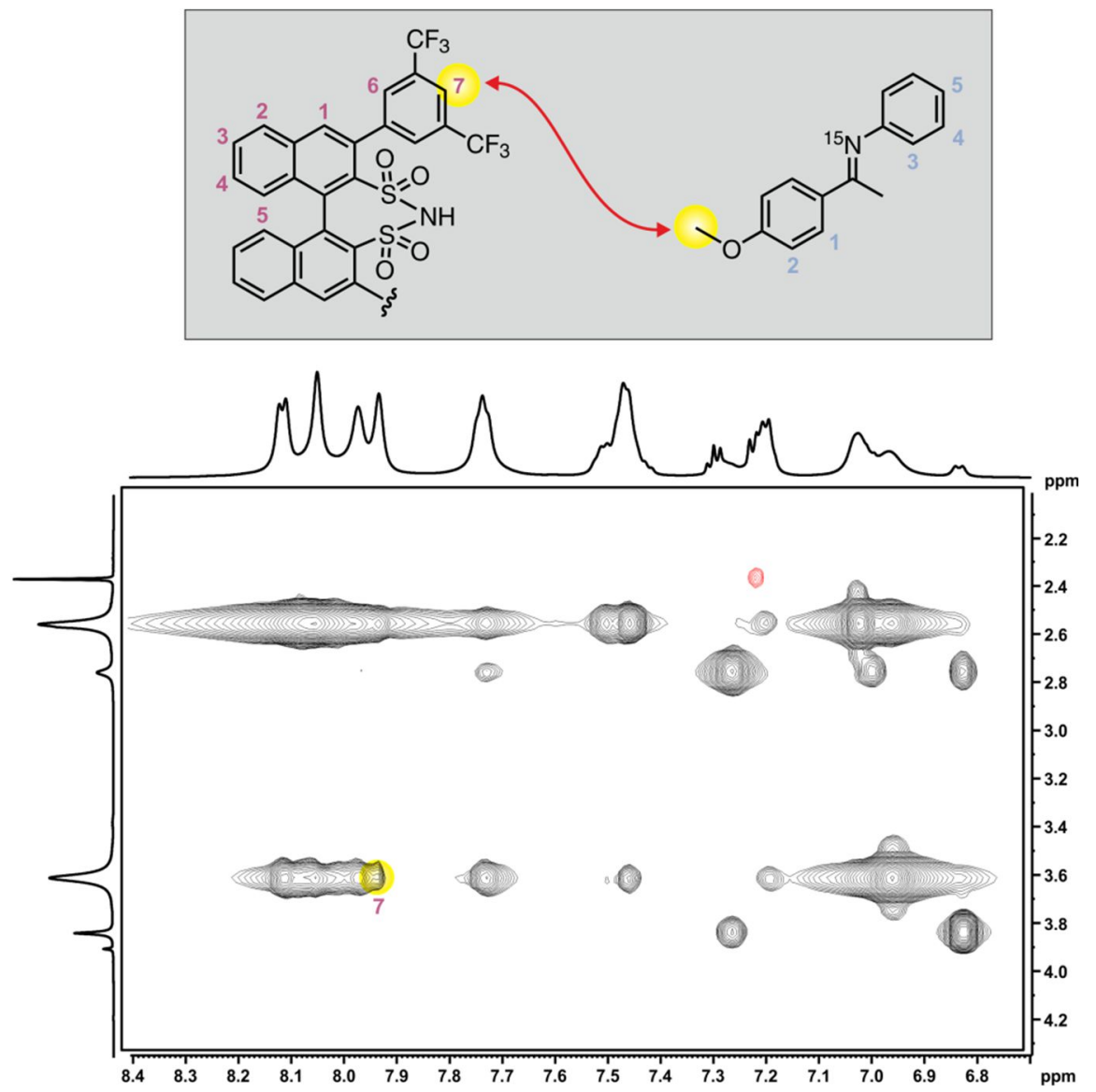

Figure S 23: A 2D ${ }^{1} \mathrm{H},{ }^{1} \mathrm{H}$ NOESY spectrum $\left(\tau_{\text {mix }}=300 \mathrm{~ms}\right.$ ) of complex $1 \mathrm{e} / 2 \mathrm{a}$ at $180 \mathrm{~K}$ in $\mathrm{CD}_{2} \mathrm{Cl}_{2}$. The NOE (labeled in yellow) between proton 7 of the DSI (purple) and the p-methoxy-group of the imine suggest the existence of type IIEO. The calculated distances between these groups are in type $E_{N}(7.65 \AA)$ are significantly bigger than in type IIEO $(3.51 \AA)$. In Figure $\mathrm{S} 18$ this NOE is shown in green. 


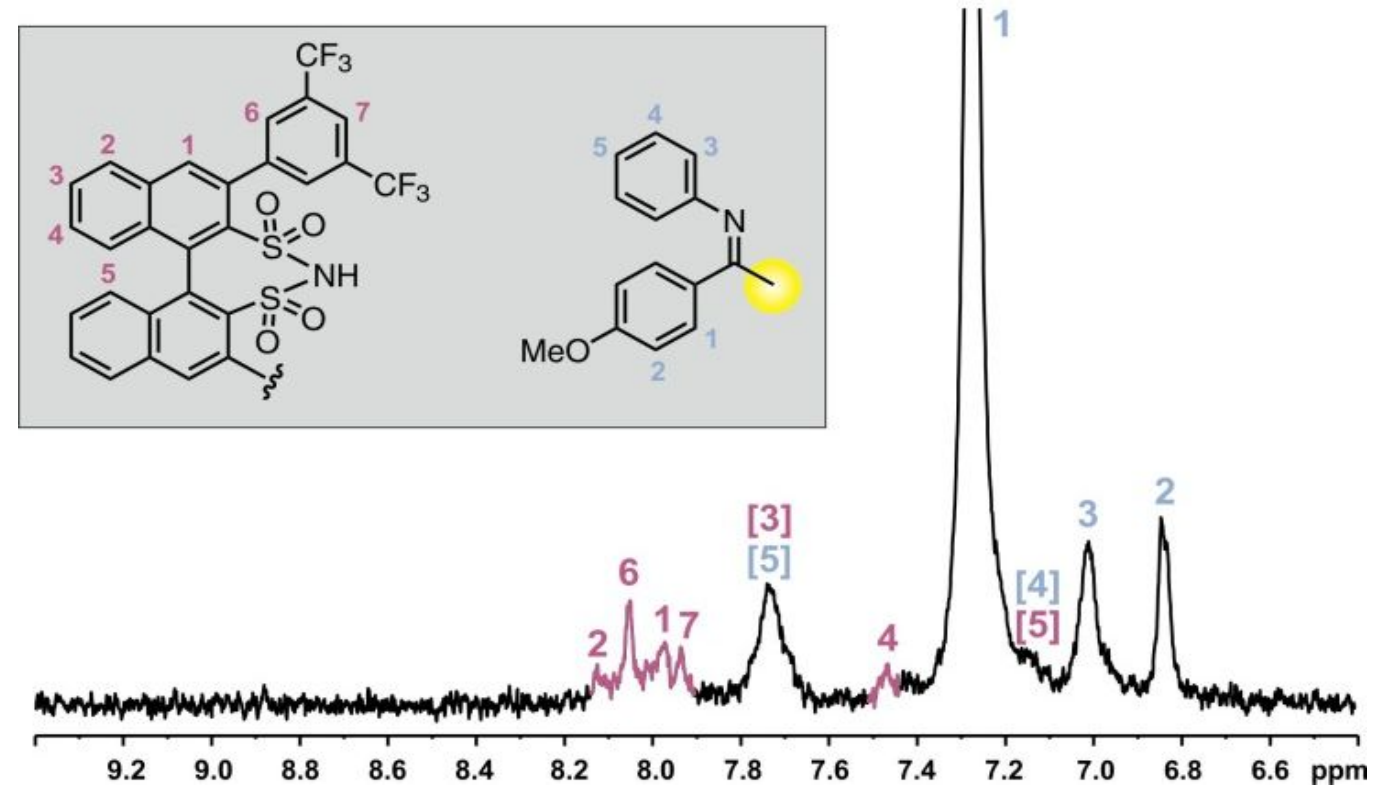

Figure S 24: A section of a selective 1D ${ }^{1} \mathrm{H},{ }^{1} \mathrm{H}-\mathrm{NOESY}\left(\tau_{\text {mix }}=100 \mathrm{~ms}\right.$, Gauss 180 pulse $)$ excited on the $\alpha$-methyl group of Z-2a (yellow circle) at $180 \mathrm{~K}$ in $\mathrm{CD}_{2} \mathrm{Cl}_{2}$ is shown. NOEs are visible to the complete catalyst backbone. Due to the extreme motion of the imine inside the binary complex no experimentally determination of the existing structures of the Z-complexes was possible. The signals of the protons in brackets are overlapping.
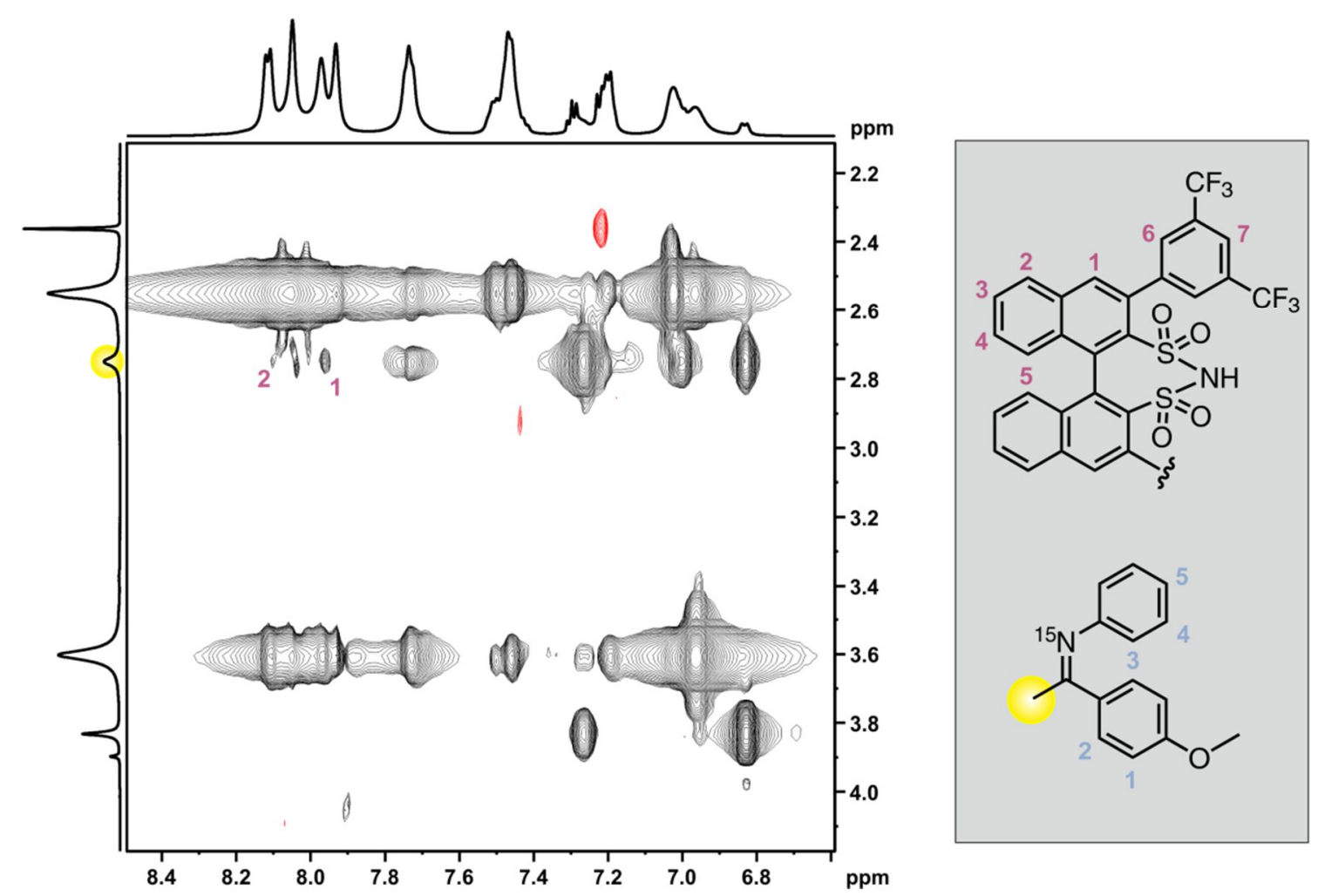

Figure S 25: $2 \mathrm{D}{ }^{1} \mathrm{H},{ }^{1} \mathrm{H}$ NOESY spectrum ( $\tau_{\text {mix }}=300 \mathrm{~ms}$ ) of complex 1e/2a at $180 \mathrm{~K}$ in $\mathrm{CD}_{2} \mathrm{Cl}_{2}$. The slight NOEs between the a-methyl-group of the imine (labeled in yellow) and some protons of the backbone of the catalyst (purple 1 and 2 ) give a hint for the existence of type $I Z_{N}$. On the other hand the experimental proof of type $I I Z_{N}$ was not possible. 

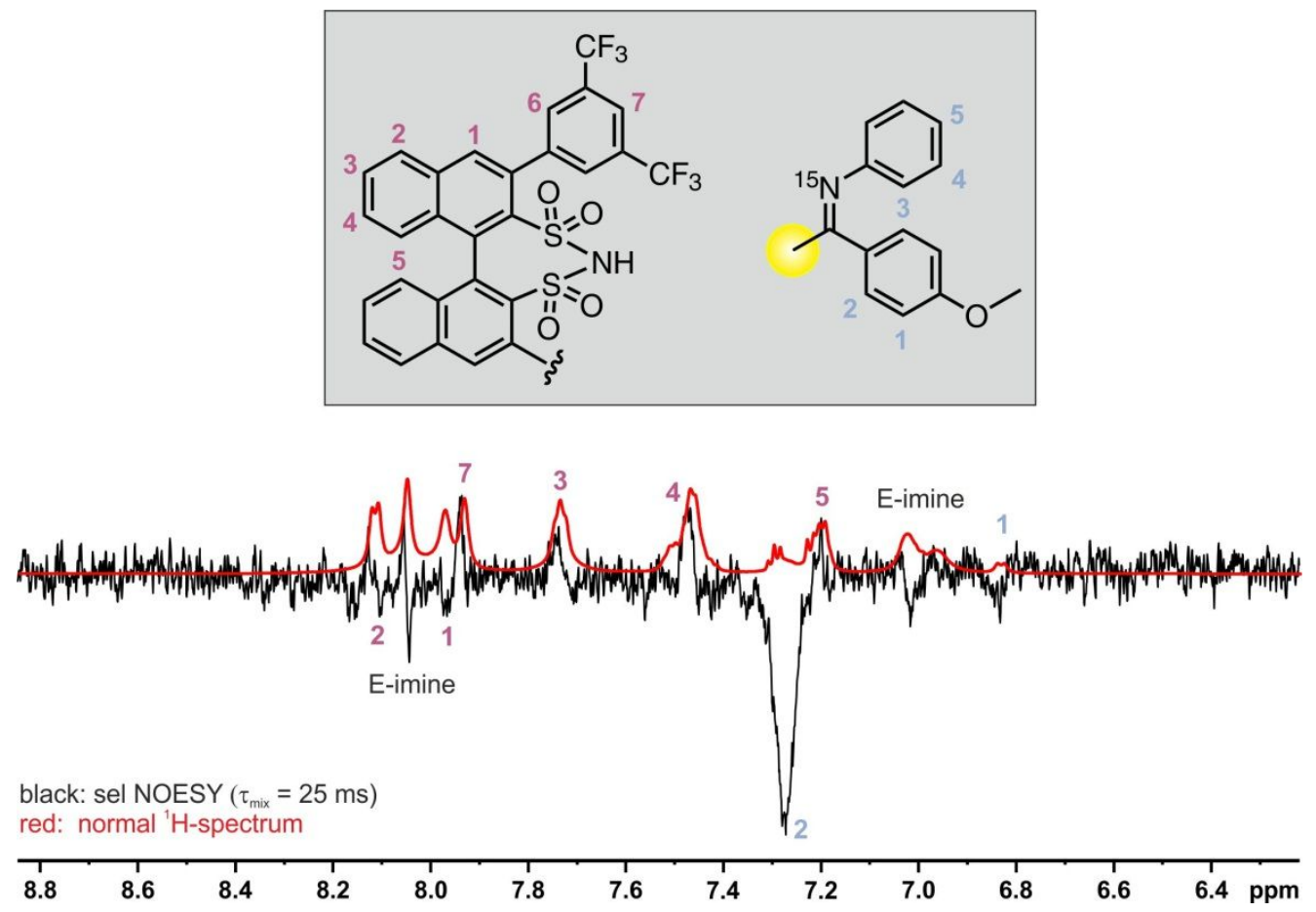

Figure S 26: A section of a selective 1D ${ }^{1} \mathrm{H},{ }^{1} \mathrm{H}-\mathrm{NOESY}\left(\tau_{\mathrm{mix}}=25 \mathrm{~ms}\right.$, Gauss 180 pulse) excited on the $\alpha$-methyl group of $\mathrm{Z-2a}$ (yellow circle) at $180 \mathrm{~K}$ in $\mathrm{CD}_{2} \mathrm{Cl}_{2}$ is shown. NOEs to the most protons of the catalyst backbone can be obtained. Due to the high excess of the binary E-complex it was not possible to excite exclusively the a-methyl group of $Z$-imine. Potentially also small parts of the a-methyl group of E-imine were excited. Therefore also the NOEs to the aromatic protons of the $E$-imine are visible.

\subsection{CF $\mathrm{CF}_{3}-\mathrm{DSI} 1 \mathrm{f} / 2 \mathrm{a}\left(\mathrm{CD}_{2} \mathrm{Cl}_{2}, 180 \mathrm{~K}\right)$}
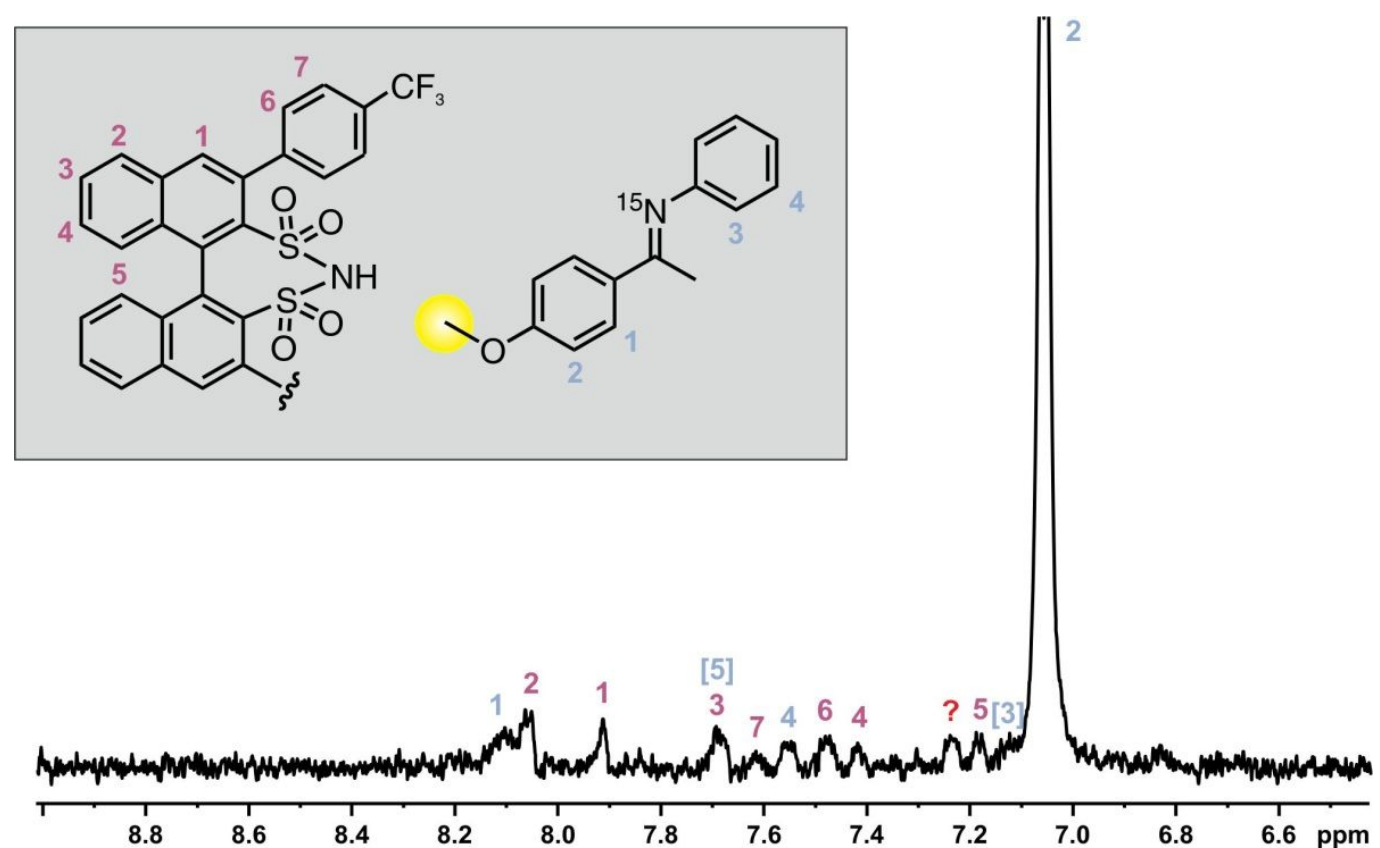

Figure S 27: A section of a selective 1D ${ }^{1} \mathrm{H}-\mathrm{NOESY}\left(\tau_{\text {mix }}=100 \mathrm{~ms}\right.$, RE-Burp pulse $)$ excited on the $p$-methoxy group of $E-2 a$ (yellow circle) at $180 \mathrm{~K}$ in $\mathrm{CD}_{2} \mathrm{Cl}_{2}$ is shown. NOEs are visible to the complete catalyst backbone, indicating the existence of type $E_{N}$. However, also for some of the less populated type $E_{O}$-orientations (e.g. type $\left.I E_{O}\right)$ NOEs between the $p$-methoxy group of $E-2 a$ (yellow circle) and the BINOL backbone were expected. But in analogy the Figure $S 21$ the strong NOEs to the protons 1 and 2 of the backbone (purple) indicate the existence auf type $E_{N}$. Nevertheless the sterically less demanding 3,3'-substituents of the catalyst seems to enable a higher mobility of the imine in the complex. Therefore the intensity to the NOE to the proton 2 of the backbone (purple) is increased compared to the $\left(\mathrm{CF}_{3}\right)_{2}$-DSI 1e/2a-complex. The signals of the protons in brackets are overlapping. The signal marked with a red ? might be from toluene ( $E$-complex : Z-complex : toluene $=7.6: 1: 1$ in the investigated sample). 

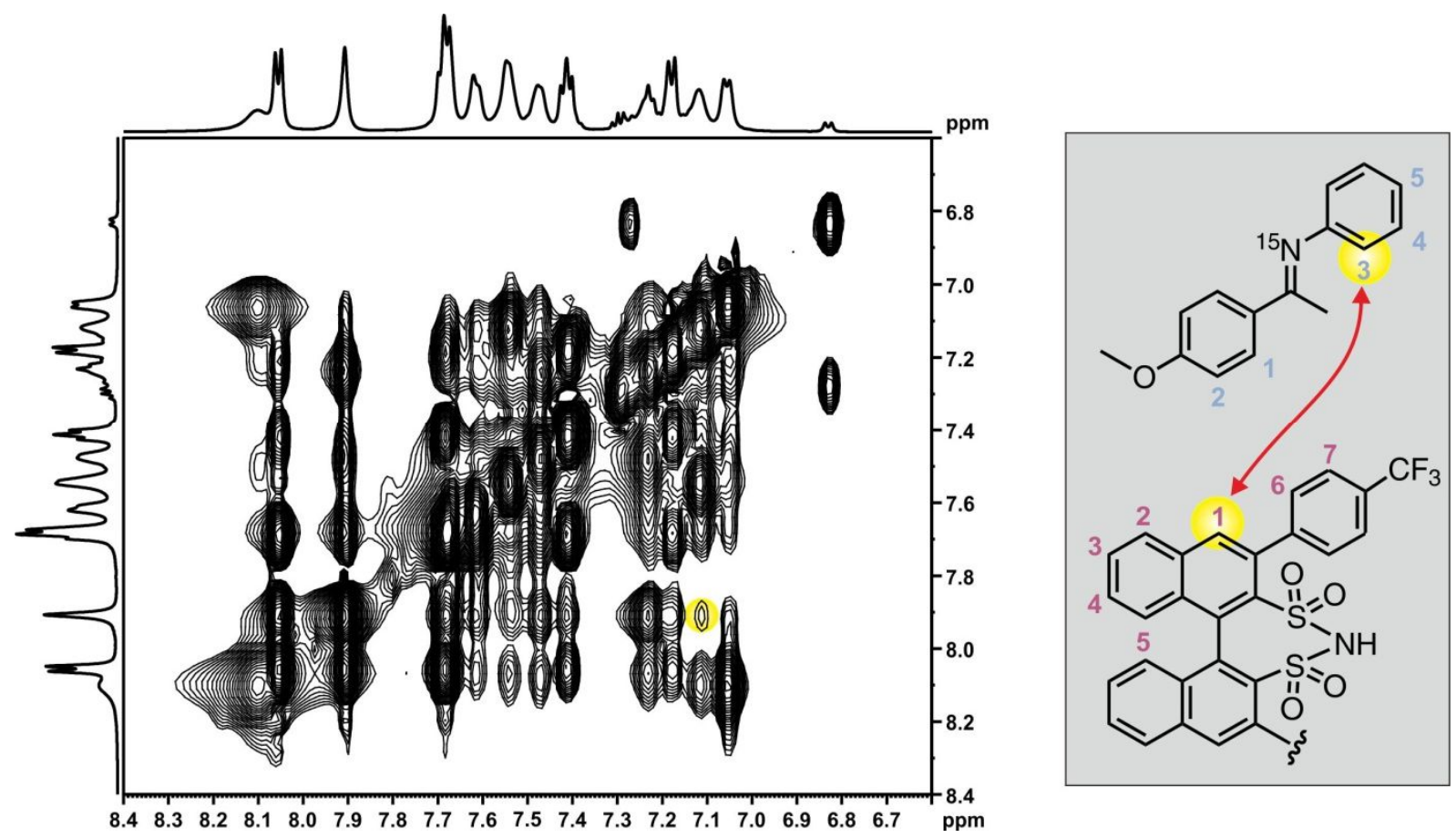

Figure S 28: $2 \mathrm{D}{ }^{1} \mathrm{H},{ }^{1} \mathrm{H}$ NOESY spectrum $\left(\tau_{\text {mix }}=300 \mathrm{~ms}\right.$ ) of complex $1 \mathrm{f} / 2 \mathrm{a}$ at $180 \mathrm{~K}$ in $\mathrm{CD}_{2} \mathrm{Cl}_{2}$. In contrast to the CPAcomplexes the NOE between proton 1 of the DSI (purple) and proton 3 of the imine (blue) does not identify type IIEO (labeled in yellow) unambiguously.

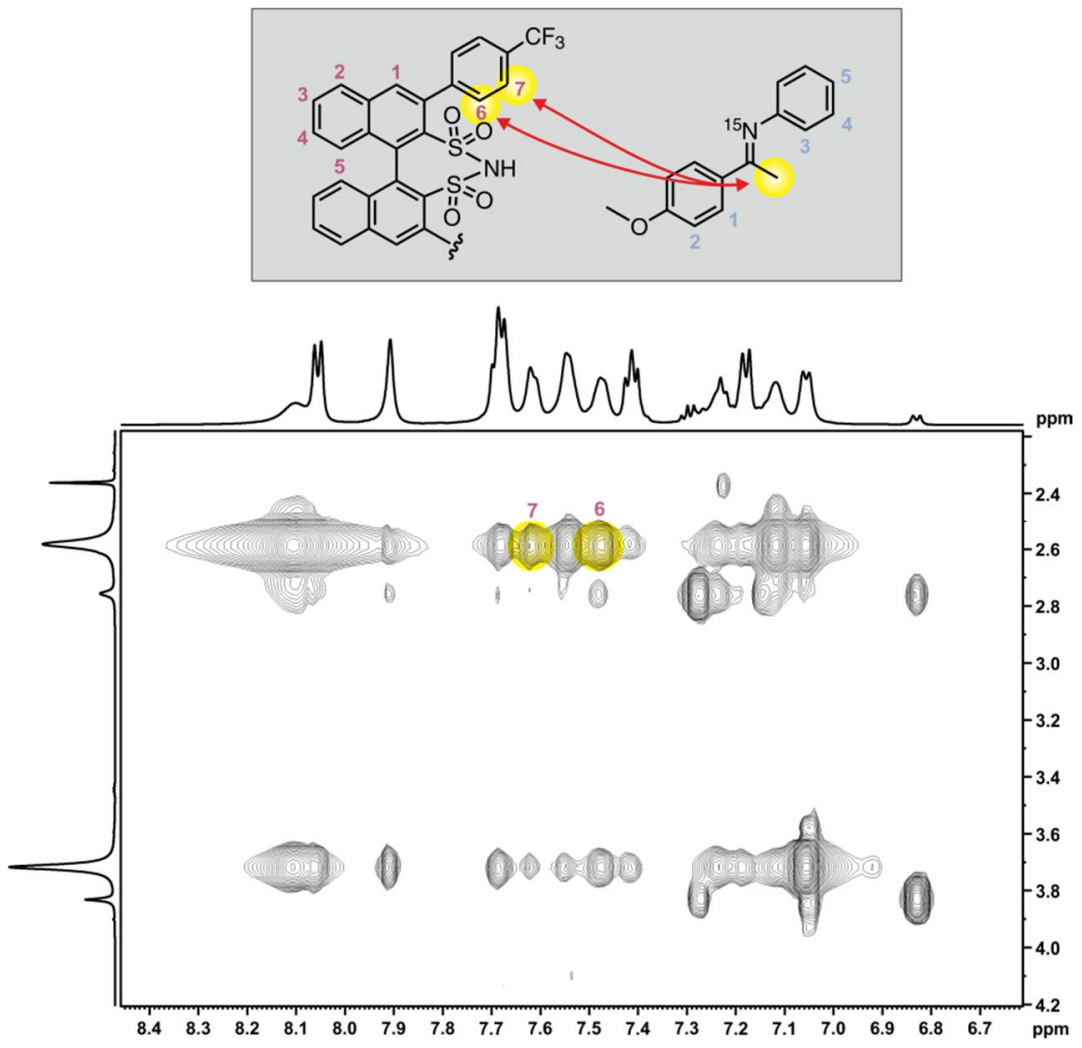

Figure S 29: A 2D ${ }^{1} \mathrm{H},{ }^{1} \mathrm{H}$ NOESY spectrum $\left(\tau_{\text {mix }}=300 \mathrm{~ms}\right.$ ) of complex $1 \mathbf{f} / \mathbf{2 a}$ at $180 \mathrm{~K}$ in $\mathrm{CD}_{2} \mathrm{Cl}_{2}$. The NOE (labeled in yellow) between the 3,3'-substituents of the DSI (purple 6 and 7) and the p-methoxy-group of the imine suggest the existence of type $I I E_{O}$. These assumption is based on the calculated distances between these groups are for the 1e/2acomplex (see green NOE in Figure S 18). 


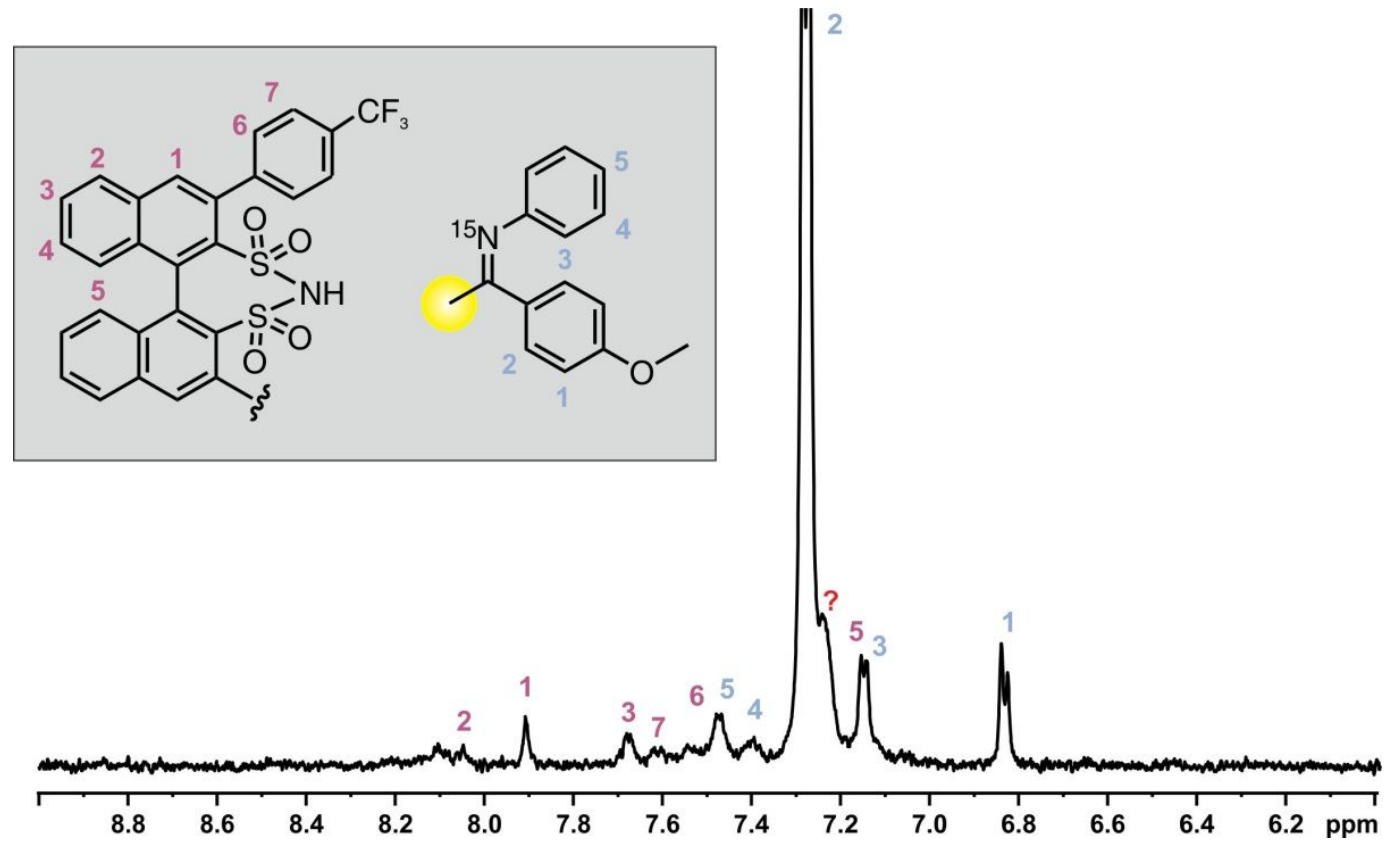

Figure S 30: A section of a selective 1D ${ }^{1} \mathrm{H},{ }^{1} \mathrm{H}$-NOESY $\left(\tau_{\text {mix }}=100 \mathrm{~ms}\right.$, Gauss 180 pulse $)$ excited on the $\alpha$-methyl group of Z-2a (yellow circle) at $180 \mathrm{~K}$ in $\mathrm{CD}_{2} \mathrm{Cl}_{2}$ is shown. NOEs are visible to the complete catalyst backbone. Due to the extreme motion of the imine inside the binary complex no experimentally determination of the existing structures of the $Z$-complexes was possible. The signal marked with a red ? might be from toluene. Therefore, that the excited a-methyl group of Z-2a is close to the methyl-group of toluene it might also be excited ( $E$-complex : Z-complex : toluene $=7.6: 1: 1$ in the investigated sample).
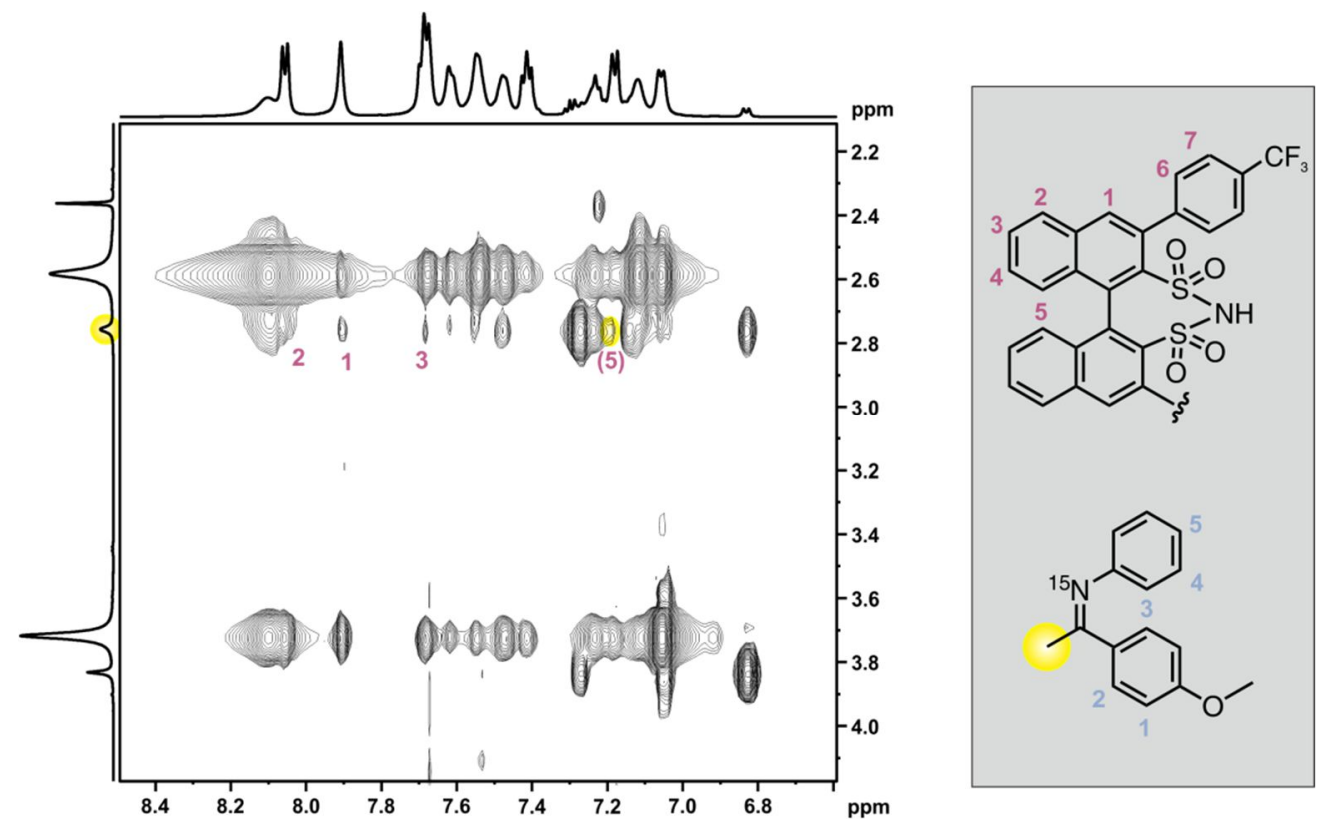

Figure S 31: $2 \mathrm{D}{ }^{1} \mathrm{H},{ }^{1} \mathrm{H}$ NOESY spectrum $\left(\tau_{\text {mix }}=300 \mathrm{~ms}\right.$ ) of complex $1 \mathbf{f} / 2 \mathrm{a}$ at $180 \mathrm{~K}$ in $\mathrm{CD}_{2} \mathrm{Cl}_{2}$. The slight NOEs between the a-methyl-group of the imine (labeled in yellow) and some protons of the backbone of the catalyst (purple 1, 2, 3 and 5 ) give a hint for the existence of type $I Z_{N}$. On the other hand the experimental proof of type $I I Z_{N}$ was not possible. 


\section{$8.4 C F_{3}-D S I 1 f / 2 b\left(C D_{2} C l_{2}, 180 K\right)$}
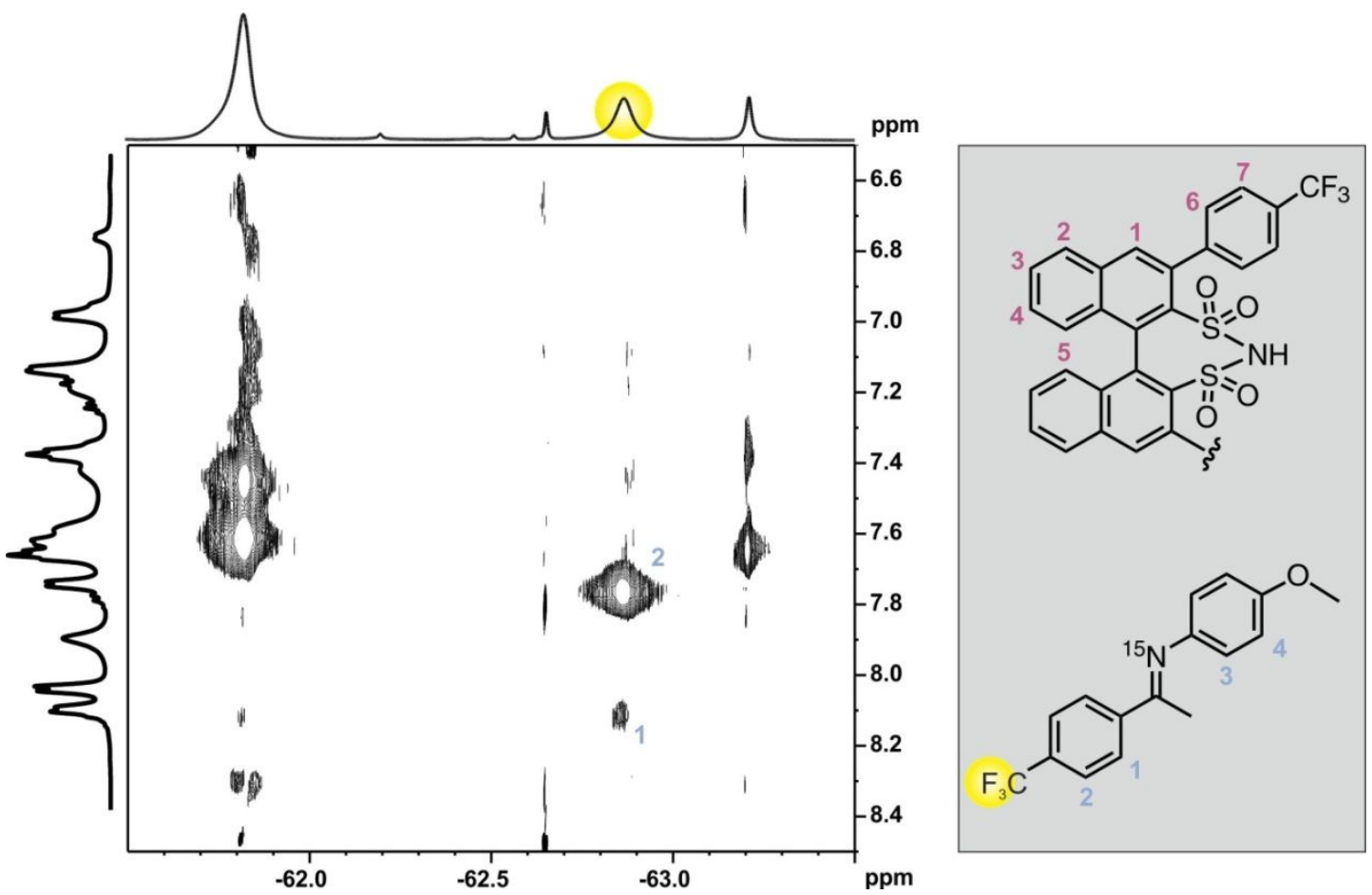

Figure S 32: The ${ }^{1} \mathrm{H},{ }^{19} \mathrm{~F}$-HOESY spectra of complex $\mathbf{1 f} / \mathbf{2 b}$ at $180 \mathrm{~K}$ in $\mathrm{CD}_{2} \mathrm{Cl}_{2}$. The trifluromethyl group of $\boldsymbol{E}$-2b (labeled in yellow) shows none of the expected signals (purple 1 and 2) to the backbone of the catalyst. Therefore this spectrum could not be used to identify type $E_{N}$.
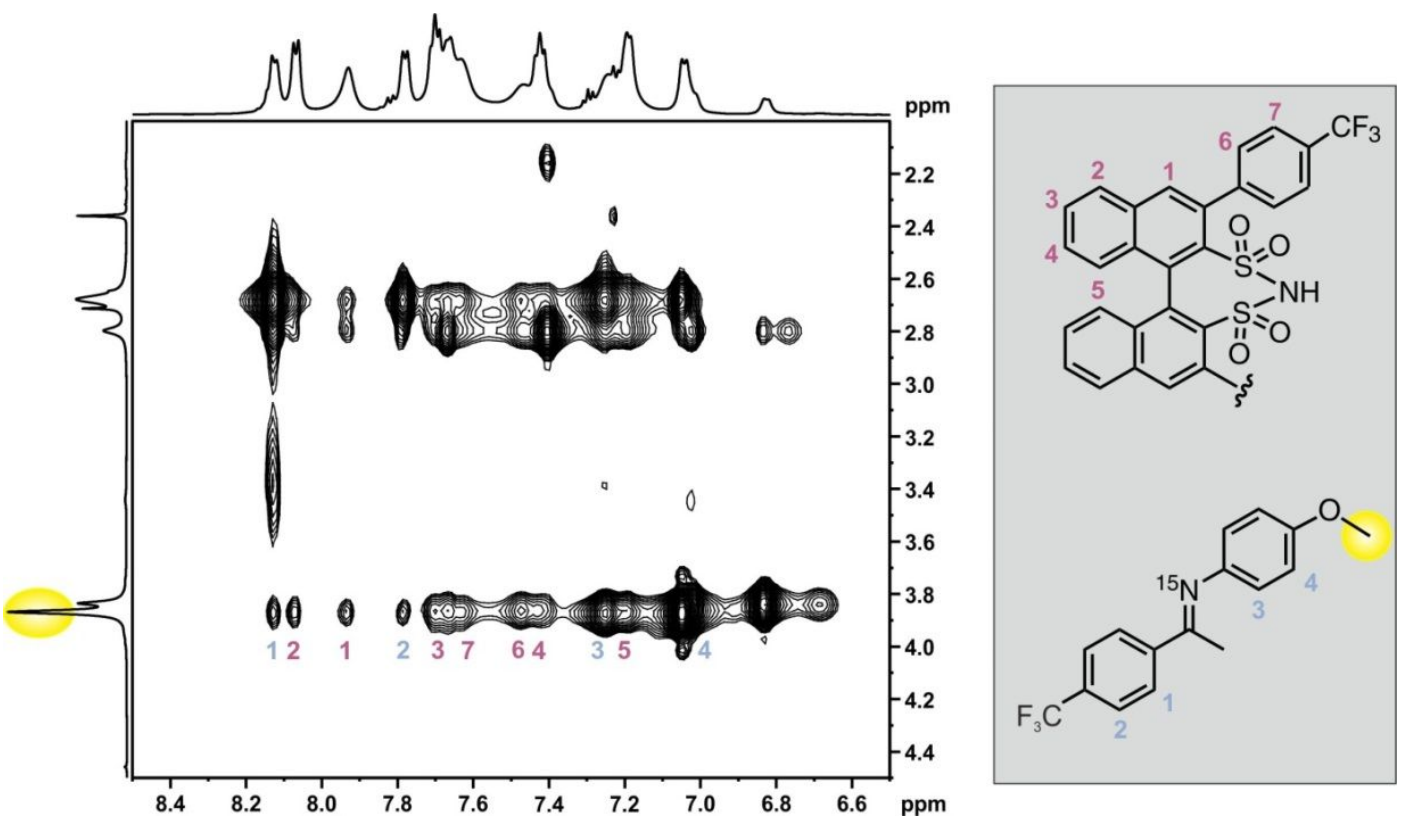

Figure S 33: $2 \mathrm{D}{ }^{1} \mathrm{H},{ }^{1} \mathrm{H}$ NOESY spectrum $\left(\tau_{\text {mix }}=300 \mathrm{~ms}\right.$ ) of complex $\mathbf{1 f} / \mathbf{2} \mathbf{b}$ at $180 \mathrm{~K}$ in $\mathrm{CD}_{2} \mathrm{Cl}_{2}$. The existence of type $\|_{\mathrm{O}}$ is assumed because of the strongest NOEs between the methoxy-group of the imine (labeled in yellow) and the catalyst-backbone (purple 3, 4 and 5). Whereas, the NOEs between the methoxy-group of the imine (labeled in yellow) and the 3,3'-substituents of the catalyst (purple 6 and 7) can be obtained from all three orientations (type $E_{N}$, type IEO and type IIEO). The measurement of a selective $1 \mathrm{D}{ }^{1} \mathrm{H},{ }^{1} \mathrm{H}-\mathrm{NOESY}$, where the $\mathbf{\alpha}$-methyl group of $E$-2 $\mathbf{b}$ is excited, is not possible due to signal-overlap. Thus, no unambiguous experimental proof for the type $E_{N}$ was found, but due to the situation in the other complexes and the computationally predicted preference for this orientation, nevertheless the existence of type $E_{N}$ was assumed. 


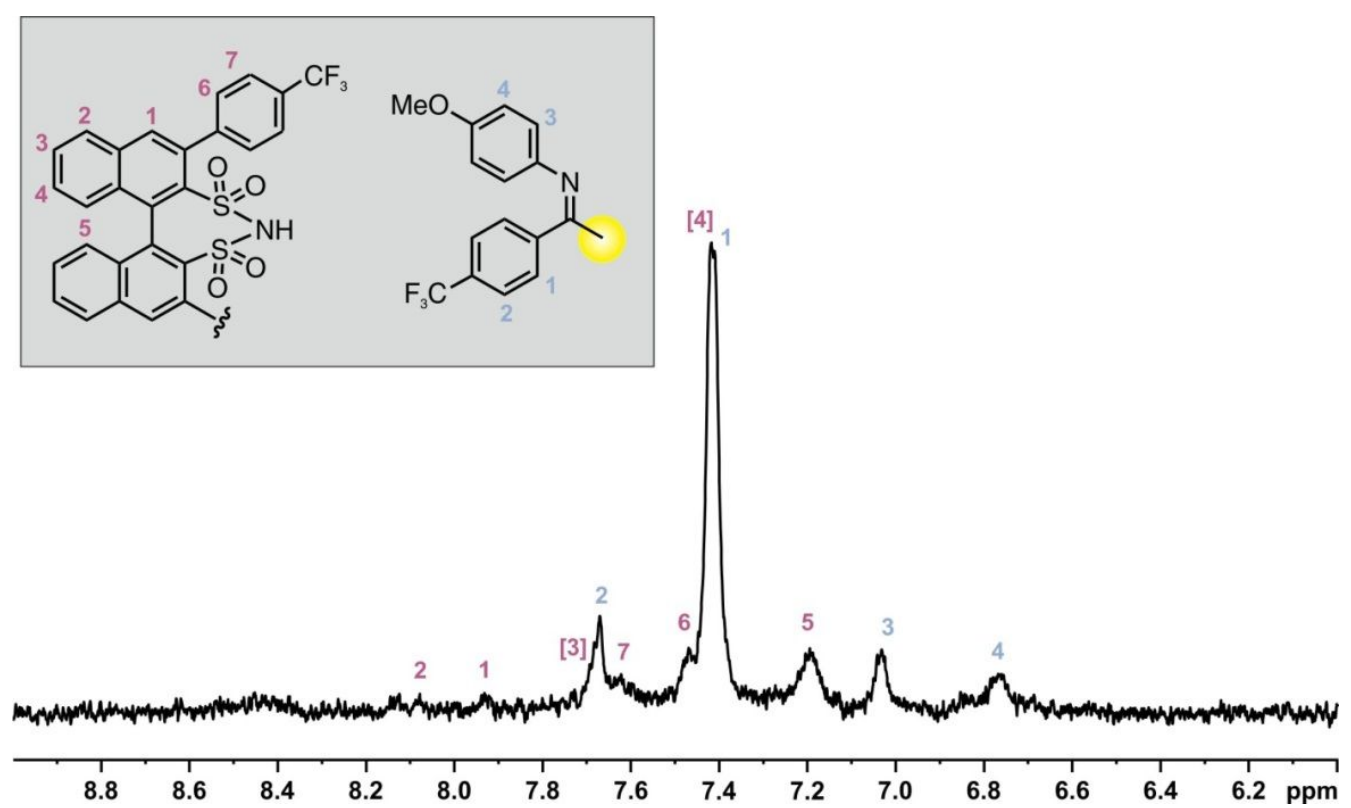

Figure S 34: A section of a selective 1D ${ }^{1} \mathrm{H},{ }^{1} \mathrm{H}-\mathrm{NOESY}\left(\tau_{\mathrm{mix}}=100 \mathrm{~ms}\right.$, Gauss 180 pulse) excited on the $\alpha$-methyl group of $Z-2 \mathbf{b}$ (yellow circle) at $180 \mathrm{~K}$ in $\mathrm{CD}_{2} \mathrm{Cl}_{2}$ is shown. Due to the extreme motion of the imine inside the binary complex no experimentally determination of the existing structures of the Z-complexes was possible. NOEs are visible to the complete catalyst backbone. The signals of the protons in brackets are overlapping.
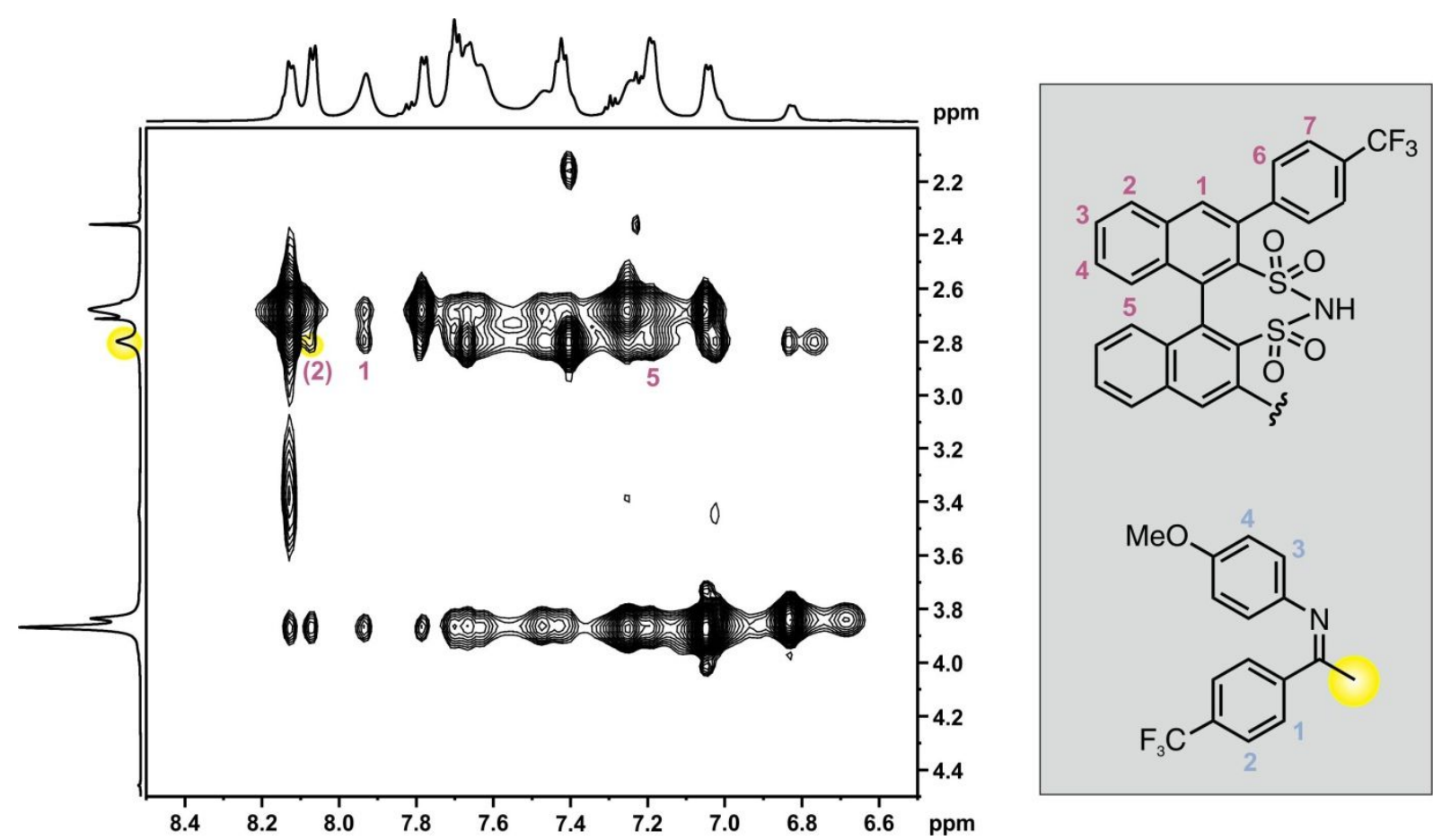

Figure S 35: 2D ${ }^{1} \mathrm{H},{ }^{1} \mathrm{H}$ NOESY spectrum $\left(\tau_{\text {mix }}=300 \mathrm{~ms}\right)$ of complex $\mathbf{1 f} / \mathbf{2} \mathbf{b}$ at $180 \mathrm{~K}$ in $\mathrm{CD}_{2} \mathrm{Cl}_{2}$. The slight NOEs between the $\alpha$-methyl-group of the imine (labeled in yellow) and some protons of the backbone of the catalyst (purple 1 , 2 and 5) give a hint for the existence of type $I Z_{N}$. 

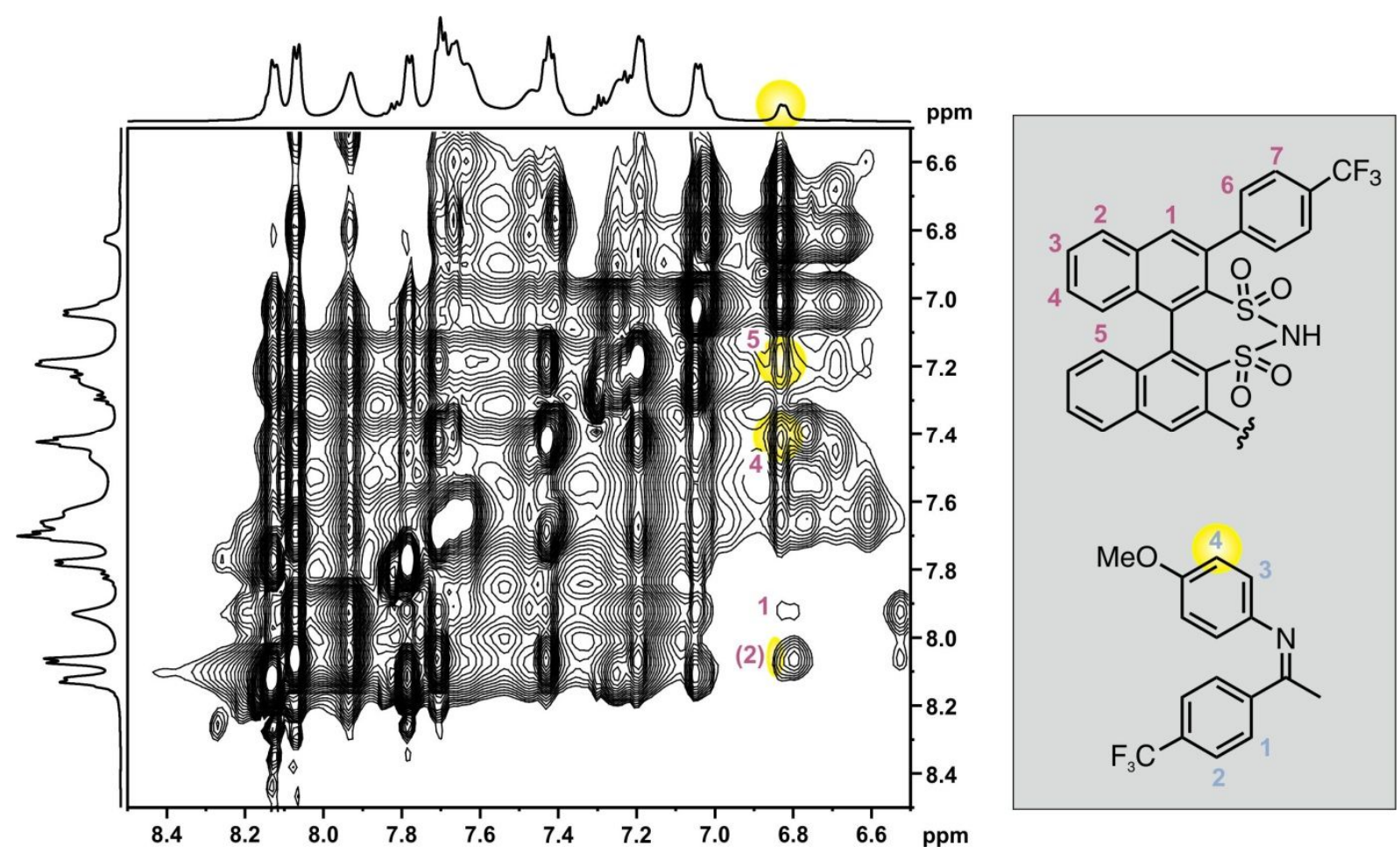

Figure S 36: $2 \mathrm{D}{ }^{1} \mathrm{H},{ }^{1} \mathrm{H}$ NOESY spectrum $\left(\tau_{\text {mix }}=300 \mathrm{~ms}\right.$ ) of complex $\mathbf{1 f} / \mathbf{2 b}$ at $180 \mathrm{~K}$ in $\mathrm{CD}_{2} \mathrm{Cl}_{2}$. The existence of type IIZN could be assumed because of the slight NOEs between the aniline moiety of the imine (blue 4) and the backbone of the catalyst (purple 1, 2, 4 and 5). 


\section{In-situ NMR Kinetics of the Transfer Hydrogenation}

Imine 2a (11.95 mg for 1e-sample or respectively $11.87 \mathrm{mg}$ for $1 \mathrm{f}$-sample, $0.05 \mathrm{mmol}, 1.0$ eq) and diethyl 1,4-dihydro-2,6-dimethyl-3,5-pyridinedicarboxylate (16.8 $\mathrm{mg}$ for 1e-sample or rather $18.7 \mathrm{mg}$ for $1 \mathrm{f}$-sample, $0.07 \mathrm{mmol}, 1.4 \mathrm{eq}$ ) were weighted directly into an NMR tube and dissolved in $0.3 \mathrm{ml}$ dry, deuterated dichloromethane. Under argon atmosphere $0.2 \mathrm{ml}$ of a catalyst stock solution ( $0.01 \mathrm{eq}, 3.01 \mathrm{mg} 1 \mathrm{e}$ in $1.5 \mathrm{~mL}$ dry $\mathrm{CD}_{2} \mathrm{Cl}_{2}$ respectively $3.83 \mathrm{mg} 1 \mathrm{f}$ in $2 \mathrm{~mL}$ dry $\mathrm{CD}_{2} \mathrm{Cl}_{2}$ ). Immediately after addition of the catalyst a ${ }^{1} \mathrm{H}$-in-situ-NMR kinetic was measured at $313.15 \mathrm{~K}$. The imine $\mathbf{2 a}$ was prepared as described in the experimental section 1.2. Both catalysts as well as Hantzsch ester were purchased from Sigma Aldrich.

For reasons of comparison, the intensity of the a-methyl group of the imine in the first spectrum was calibrated to 1 . Since for structure determination and hydrogen bond analysis dichloromethane was used, the reaction kinetics were also performed in deuterated dichloromethane. A further advantage of using dichloromethane is the better separated signals, which enable therefore a more exact integration.<smiles>CCOC(=O)C1=C(C)NC(C)=C(C(=O)OCC)C1</smiles>

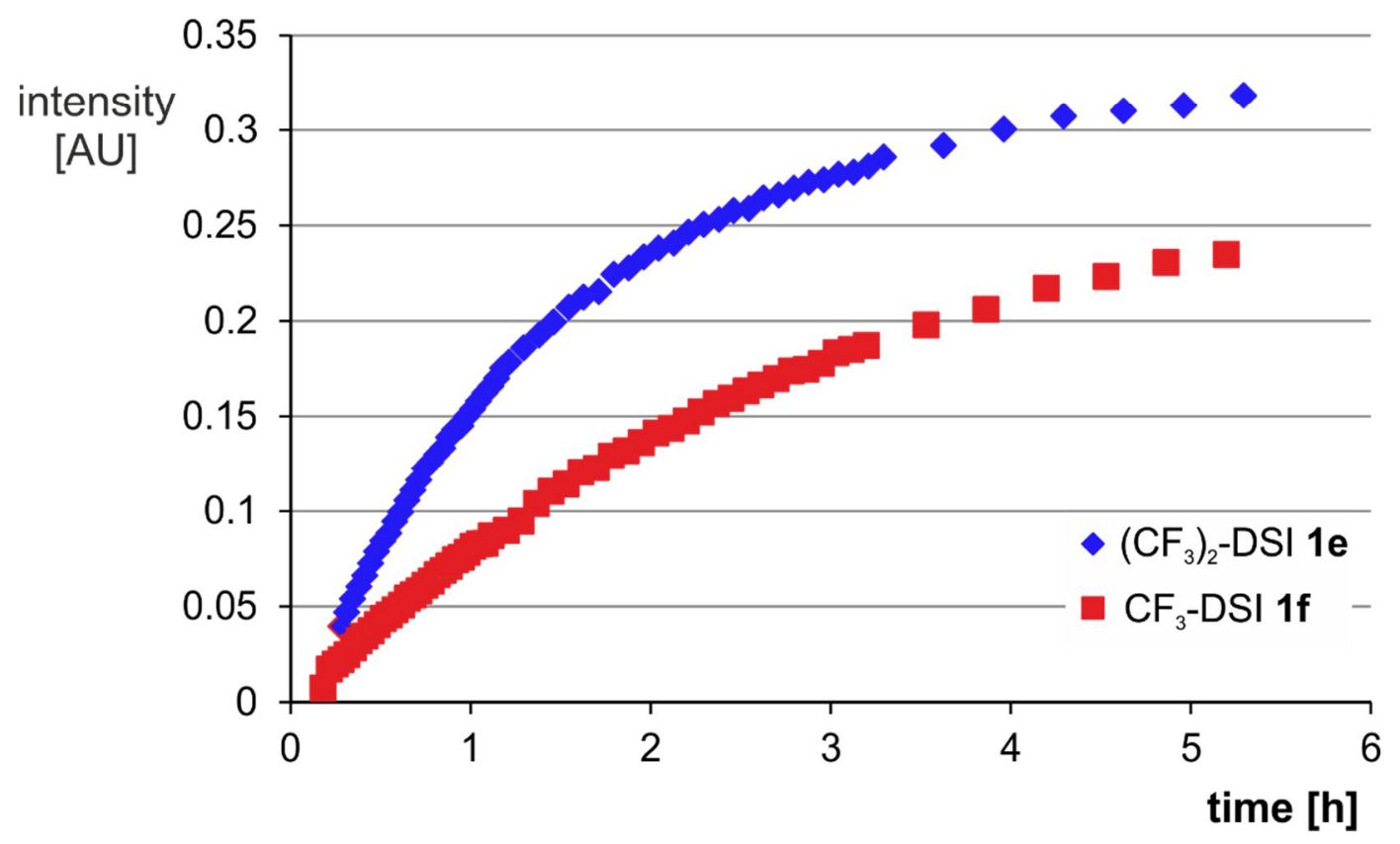

Figure S 37: The reaction profiles for the symmetric transfer hydrogenation of imine $2 \mathrm{a}$ are shown. The kinetics were done in-situ in the NMR spectrometer with 1.4 equivalents of Hantzsch ester and $1 \mathrm{~mol} \%$ catalyst $\left(\left(\mathrm{CF}_{3}\right)_{2^{-}}\right.$ DSI 1e and $\mathrm{CF}_{3}$-DSI 1f) at $40^{\circ} \mathrm{C}$ in $\mathrm{CD}_{2} \mathrm{Cl}_{2}$ 


\section{Ex-situ Reaction Kinetics of the Transfer Hydrogenation}

\subsection{HPLC chromatograms}

10.1.1 (E)-1-(4-methoxyphenyl)-N-phenylethan-1-imine 2a

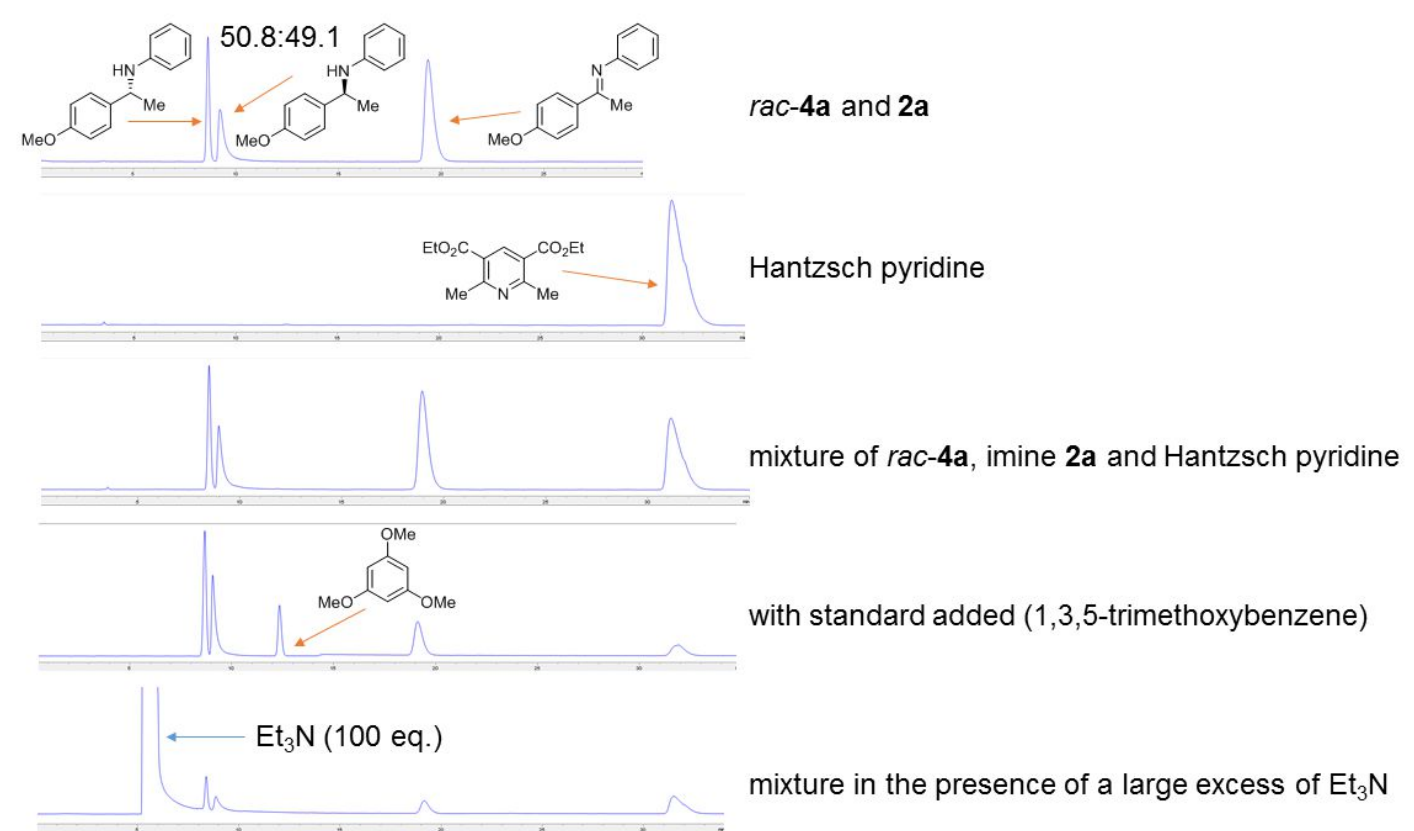

Figure S 38: CSP-HPLC chromatograms of the reaction components in the transfer hydrogenation of $\mathbf{2 a}$.

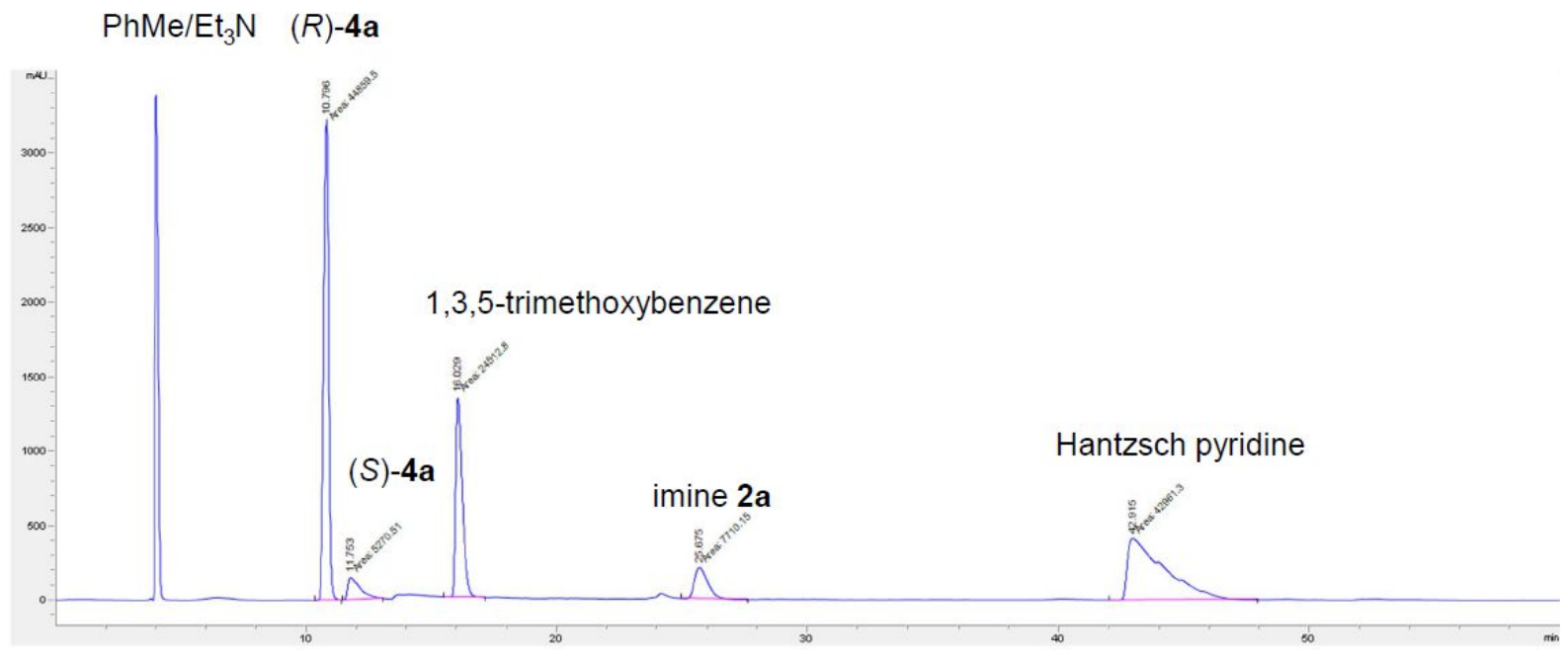

Figure S 39: Representative CSP-HPLC chromatogram of a sample taken during the transfer hydrogenation of imine 2a. HPLC conditions: Chiralpak IC, $n$-hexane/i-propanol 99:1, $0.9 \mathrm{~mL} \cdot \mathrm{min}^{-1}, 20^{\circ} \mathrm{C}, 220 \mathrm{~nm}$ detection. 


\subsubsection{N,1,1-triphenylmethanimine 2c}

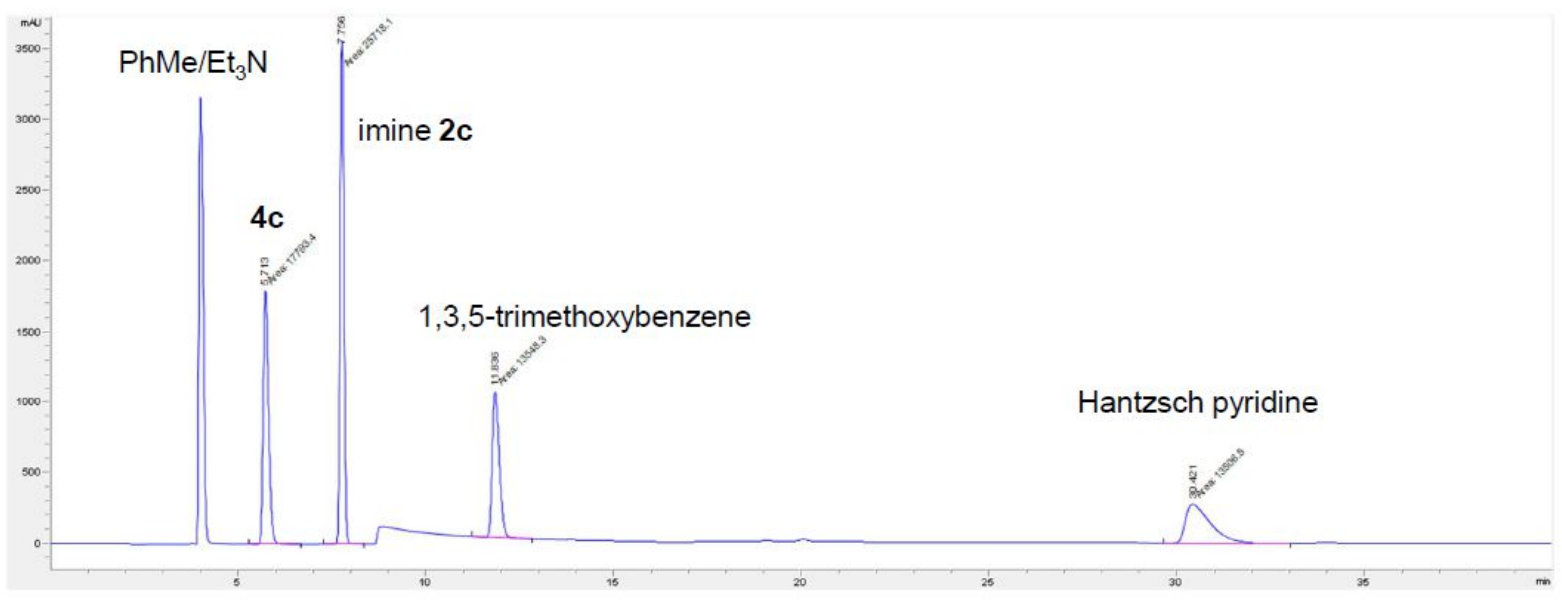

Figure S 40: Representative CSP-HPLC chromatogram of a sample taken during the transfer hydrogenation of imine 2c. HPLC conditions: Chiralpak IC, $n$-hexane/ $i$-propanol 99:1, $0.9 \mathrm{~mL} \cdot \mathrm{min}^{-1}, 20{ }^{\circ} \mathrm{C}, 220 \mathrm{~nm}$ detection.

\subsection{3 (E)-1-(4-methylphenyl)- $N$-phenylethan-1-imine $\left(98 \%{ }^{15} \mathrm{~N}\right) 2 \mathrm{~d}$}

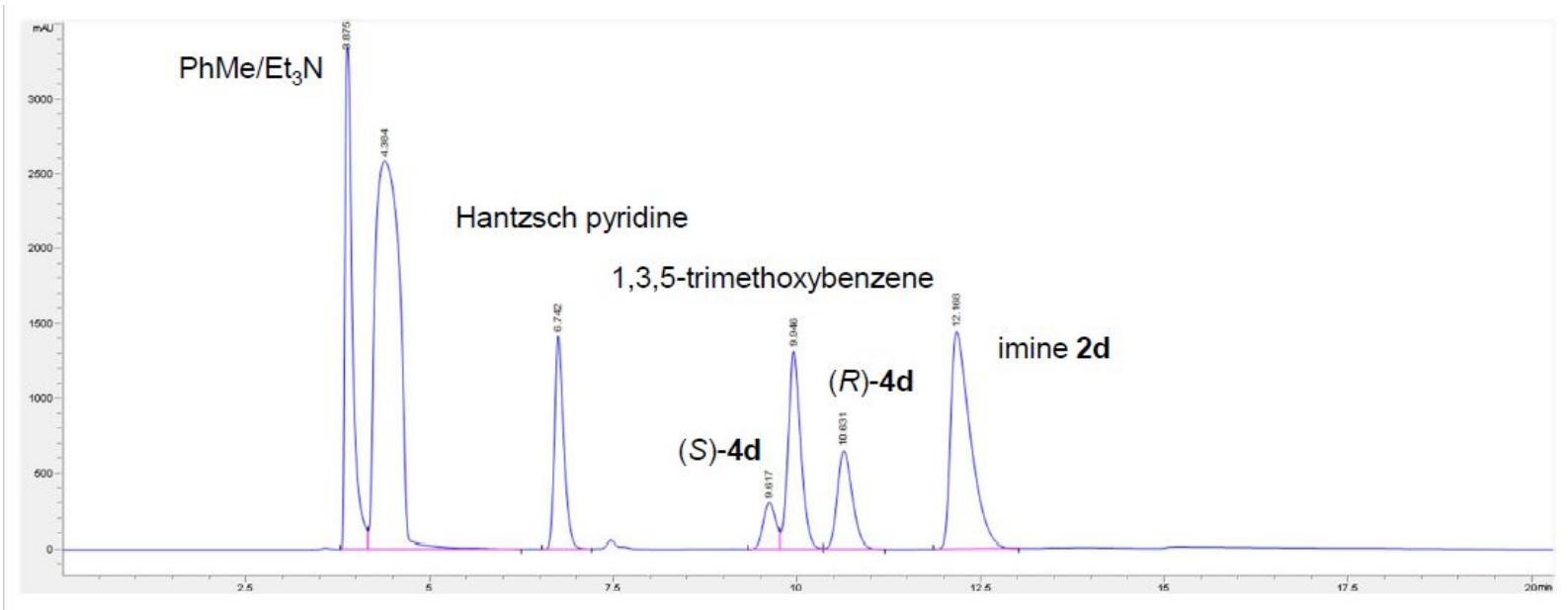

Figure S 41: Representative CSP-HPLC chromatogram of a sample taken during the transfer hydrogenation of imine 2d. HPLC conditions: Chiralcel OD-H, $n$-hexane/ $i$-propanol 98:2, $0.9 \mathrm{~mL} \cdot \mathrm{min}^{-1}, 20^{\circ} \mathrm{C}, 220 \mathrm{~nm}$ detection.

\subsection{4 (E)-1-(4-trifluoromethylphenyl)- $\mathrm{N}$-phenylethan-1-imine $\left(98 \%{ }^{15} \mathrm{~N}\right) 2 \mathrm{e}$}

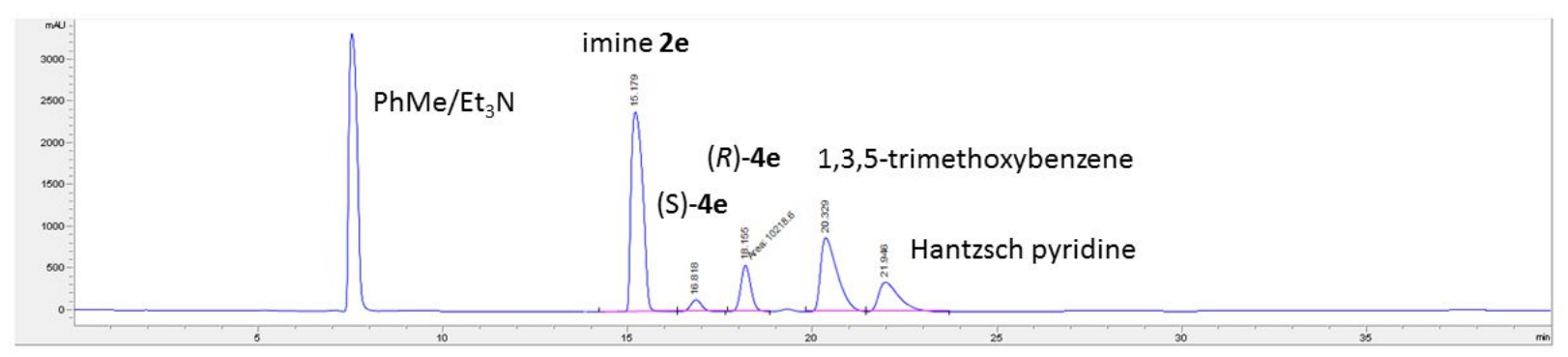

Figure S 42: Representative CSP-HPLC chromatogram of a sample taken during the transfer hydrogenation of imine 2e. HPLC conditions: Chiralpak IA, $n$-hexane/i-propanol 99:1, $0.9 \mathrm{~mL} \cdot \mathrm{min}^{-1}, 20^{\circ} \mathrm{C}, 220 \mathrm{~nm}$ detection. 


\subsection{Enantiomeric Excess with Different Catalysts}

The enantiomeric ratios of the chiral amines were determined from crude reaction mixtures by CSP-HPLC after substantial conversion to the products (usually after $2 \mathrm{~h}$ ). We observed that $e e$ is constant during the reaction.

Table S 3: The obtained ee-values for the asymmetric transfer hydrogenation in toluene at $35^{\circ} \mathrm{C}$ by using different $(R)$-catalysts are shown. The ee-values were determined from the crude reaction mixture after 120 min by chiral HPLC. [a] After isolation. See reference. ${ }^{[6]}$

\begin{tabular}{|c|c|c|}
\hline chiral amine & catalyst & ee [\%] \\
\hline \multirow{6}{*}{$\mathrm{MeO}_{4 a}$} & $(R)-\mathrm{TRIP} 1 \mathrm{a}$ & $85^{[a]}$ \\
\hline & $(R)$-TRIFP $1 \mathbf{b}$ & $74^{[a]}$ \\
\hline & $(R)-T R I M 1 d$ & 84 \\
\hline & $(R)-\left(\mathrm{CF}_{3}\right)_{2}-\mathrm{DSI} 1 \mathrm{e}$ & 78 \\
\hline & $(R)-\mathrm{CF}_{3}-\mathrm{DSI} \mathbf{1 f}$ & 70 \\
\hline & $(R)-B I N S A \quad 1 \mathrm{~g}$ & 68 \\
\hline \multirow{4}{*}{$4 \mathrm{~d}$} & $(R)-\mathrm{TRIP} 1 \mathrm{a}$ & 78 \\
\hline & $(R)-\left(\mathrm{CF}_{3}\right)_{2}-\mathrm{DSI} 1 \mathrm{e}$ & 40 \\
\hline & $(R)-\mathrm{CF}_{3}-\mathrm{DSI} \mathbf{1 f}$ & 32 \\
\hline & $(R)$-TRIP 1a & 80 \\
\hline 4 & $(R)-\left(\mathrm{CF}_{3}\right)_{2}-\mathrm{DSI} 1 \mathrm{e}$ & 56 \\
\hline
\end{tabular}

The enantioselectivity outcome for $\left(\mathrm{CF}_{3}\right)_{2}$-DSI 1e with different imines is not correlated to the Hammett parameter $\sigma_{\text {para }}$ (for MeO-, Me- and $\mathrm{CF}_{3}$-substituted imines) given that the MeOimine $2 \mathbf{a}$ gives high ee value, but not $\mathrm{CF}_{3^{-}}$or Me- substituted imines $\mathbf{2 d}$ and $\mathbf{2 e}$. For the DSI complexes $\mathbf{1 e} / \mathbf{2 a}$ and $\mathbf{1 e} / 3 \mathbf{a}$, with weaker $\mathrm{H}$-bonds and higher ion-pair contribution (e.g., internal acidity parameter $\delta_{Z}\left({ }^{15} \mathrm{~N}\right)<196 \mathrm{ppm}$ or $\left.{ }^{1} J_{\mathrm{NH}}(E)>90 \mathrm{~Hz}\right)$, high enantioselectivities are observed. In contrast, if the internal acidity parameter $\delta\left({ }^{15} \mathrm{~N}\right)$ suggests stronger H-bonds in the DSI complexes $\left(\delta_{z}>196 \mathrm{ppm}\right)$ with $\mathbf{2} \mathbf{d}$ and $\mathbf{2 e}$, as well as in the complex TRIP 1a/3a, then such complexes give low to moderate enantioselectivities.

With imine $2 \mathbf{e}$, only broad peaks were observed in the ${ }^{1} \mathrm{H}$ and ${ }^{15} \mathrm{~N}$ NMR spectrum, suggesting still fast exchange even at $180 \mathrm{~K}$. However, for the Me-imine $\mathbf{2 d}$, we observed sharp peaks, and slightly higher amount of Z-imine complex in the ground state than for the MeO-imine $2 a$ (28\% vs. $17 \%$, respectively). The ee values are thus probably dependent only on the combination of transition states and their relative energies (from both $E$ - and Z-imines) and not on the ground state populations (Curtin-Hammett). In this case, applying DTS was not applicable to DSI, because DSI is decomposing under illumination at $365 \mathrm{~nm}$. Another possibility for the deviating enantioselectivities is aggregation. However, in the spectra only one set of signals is observable even at extremely low temperature. Thus, the reduced $\mathrm{H}$ bond strength in DSI seems to facilitate fast exchange on the NMR time scale. 


\subsection{Reactivity Analysis}

\subsubsection{1-(4-methoxyphenyl)-N-phenylethan-1-amine 4a}

From the position of the data points of the DSI-complexes on the Steiner-Limbach curve (Figure $3 \mathrm{~b}$ ) it is obvious, that both $\mathrm{DSI} / \mathbf{2 a}$-complexes form weaker hydrogen bonds than the CPA-complexes. On the left side of the curve, a weaker hydrogen bond denotes a more acidic proton donor, i.e. a more complete proton transfer. ${ }^{[2]}$ By comparison of the exact ${ }^{15} \mathrm{~N}$ chemical shifts in the DSI/2a-complexes (for values see $\mathrm{SI}$ ), it is apparent that the $\left(\mathrm{CF}_{3}\right)_{2}$-DSI $1 \mathrm{e}$ is just slightly more acidic than the $\mathrm{CF}_{3}$-DSI $1 \mathrm{f}$ (Figure $3 \mathrm{~b}$ ). Previously, we have shown that for the CPA-catalyzed transfer hydrogenation, the least acidic TRIP (i.e. forming the strongest hydrogen bond) shows the fastest overall reaction rate. ${ }^{[2]}$ Based on this observation, we expected the reactions with the DSIs $1 \mathrm{e}$ and $1 \mathrm{f}$ to be the slowest. However, the transfer hydrogenation of imine $2 \mathrm{a}$ and $\left(\mathrm{CF}_{3}\right)_{2}$-DSI $1 \mathrm{e}$ (calculated $\mathrm{pK} \mathrm{K}_{\mathrm{a}} 0.05$ in DMSO) ${ }^{[7]}$ is the fastest, whereas with $\mathrm{CF}_{3}$-DSI 1f and TRIP 1a (calc. $\mathrm{pK}_{\mathrm{a}} 3-4$ in DMSO) the overall reaction rate is similar (Figure S43). Nevertheless, the small differences in the internal acidity of the two DSIs should not reflect this huge reactivity differences. This unexpected discrepancy seems to be due to the reduced influence of the hydrogen bond as a structural anchor by using more acidic DSI-catalysts. On the other hand, the considerable difference in acidity between CPAs and DSIs should not result in similar reactivity of TRIP $1 \mathrm{a}$ and $\mathrm{CF}_{3}$-DSI 1f, if also in the extended $p K_{a}$ range a direct correlation between the acidity and reactivity exists. The even slower reaction rate of TRIM (1d), which has smaller substituents (large steric parameter AREA $\theta$ 61), ${ }^{[8]}$ showing the importance of the bulky 3,3'-substituents and dispersion effects for the reaction rate (cf. 1a AREA $\theta$ 51). Additionally, the transfer hydrogenation was carried out with an extremely acidic, but structural similar BINOL-derived sulfonic acid BINSA $1 \mathrm{~g}$ (calc. $\mathrm{p} K_{\mathrm{a}}-9$ in DMSO). Surprisingly the reaction was extremely slow, and the overall reaction rate was similar to TRIFP (Figure S43). This observation might be explained by the low tendency of BINSA $1 \mathrm{~g}$ to form the ternary complex due to the internal stabilization by a hydrogen bond, resulting in the lack of a proper bifunctional moiety. Reaction with TiPSY (1c) is extremely slow and only traces of the product were detected after several hours, probably because of very bulky 3,3'-substitutents (small AREA $\theta$ 29).

Thus, no correlation between reactivity and acidity of catalysts with different acidic functional groups was found. In conclusion, these results suggest that the previously established acidity/reactivity-relationship ${ }^{[2]}$ is only applicable if the crucial catalytic sites of the catalyst are not changed. 
<smiles>COc1ccc(/C(C)=N/c2ccccc2)cc1</smiles>

$0.327 \mathrm{mmol}$<smiles>CCOC(=O)C1=C(C)NC(C)=C(C(=O)OCC)C1</smiles>

$1.4 \mathrm{eq}$

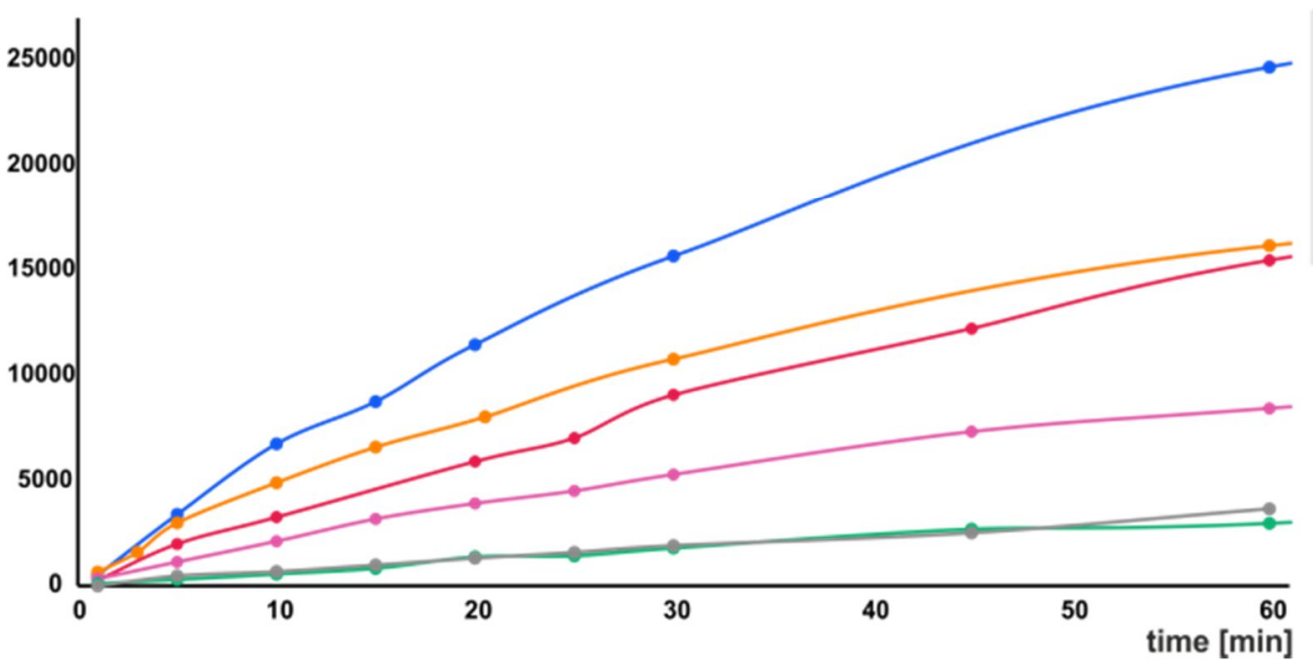<smiles>CCOC(=O)c1cc(C(=O)OCC)c(C)nc1C</smiles>

- TRIM 1d

$\rightarrow$ TRIFP $1 \mathrm{~b}$

$\rightarrow\left(\mathrm{CF}_{3}\right)_{2}-\mathrm{DSI} 1 \mathrm{e}$

$\rightarrow \mathrm{CF}_{3}$-DSI $1 \mathrm{f}$

$\rightarrow$ BINSA 1g

Figure S 43. The reactivities of the asymmetric transfer hydrogenation of imine $2 \mathrm{a}$ are shown. The reaction profiles of three CPAs $\mathbf{1 a}, \mathbf{1} \mathbf{b}$ and $1 \mathbf{d}^{[2]}$ are compared with the more acidic DSIs $1 \mathbf{e}$ and $\mathbf{1 f}$. Additionally, the even more acidic BINSA $1 \mathrm{~g}$ was used as a catalyst. The kinetics were done ex-situ and the samples analyzed by CSPHPLC. All reactions were done in a $0.327 \mathrm{mmol}$ scale with 1.4 equivalents of Hantzsch ester and $0.9 \mathrm{~mol} \%$ catalyst at $35^{\circ} \mathrm{C}$ in toluene.

For imine $\mathbf{2 d}$, the same order of reactivity was found for the three investigated catalysts (TRIP 1a and both DSIs 1e and 1f, see chapter 10.3.3). However, for imine 2c, the fastest reaction was observed with $\mathrm{CF}_{3}$-DSI 1f, suggesting that the steric requirements of the bulkier imine 2c can change the reactivity order (see chapter 10.3.2). Thus, not only the structure of the catalyst (size of the binding pocket ${ }^{[2]}$ and acidic motif) is crucial for the reactivity, but also the properties of substrate should not be neglected. 


\subsubsection{N-benzhydryl aniline 4c}<smiles>c1ccc(N=C(c2ccccc2)c2ccccc2)cc1</smiles>

2c $0.327 \mathrm{mmol}$<smiles>CCOC(=O)C1=C(C)NC(C)=C(C(=O)OCC)C1</smiles>

$1.4 \mathrm{eq}$ $\underset{\substack{\text { toluene } \\ 35^{\circ} \mathrm{C}}}{\stackrel{0.9 \mathrm{~mol} \% \text { catalyst } 1}{\longrightarrow}}$<smiles>c1ccc(NC(c2ccccc2)c2ccccc2)cc1</smiles>

$4 \mathrm{c}$

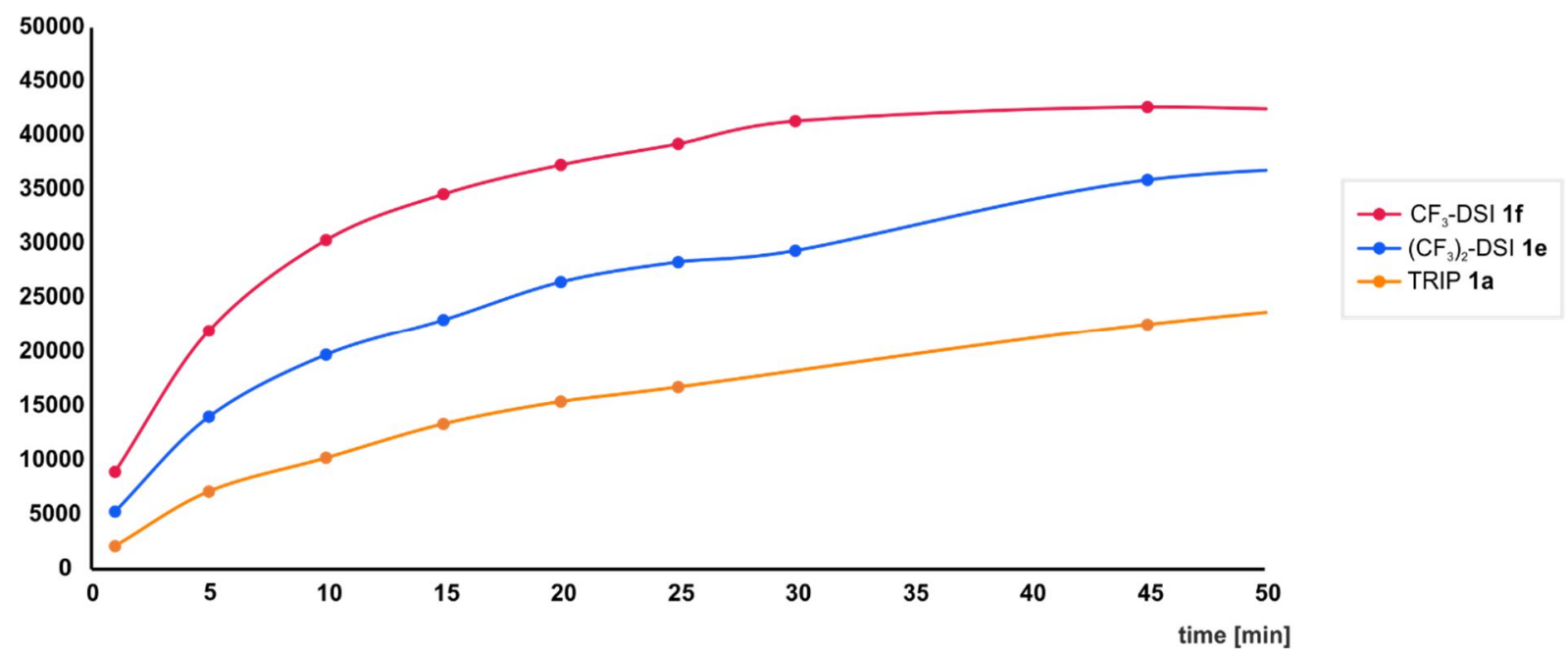

Figure S 44: The formation of $4 \mathrm{c}$ in the asymmetric transfer hydrogenation of imine 2c is shown. The kinetics were done ex-situ and the samples analyzed by CSP-HPLC. The reaction was conducted with 1.4 equivalents of Hantzsch ester and 0.9 mol \% catalyst with 0.5 eq. 1,3,5-trimethoxybenzene as the standard at $35^{\circ} \mathrm{C}$ in $\mathrm{PhMe}$.

Sulfonylimide catalyst $\left(\mathrm{CF}_{3}\right)_{2}$-DSI (1e) gives faster reaction than phosphoric acid TRIP (1a). However, in this case the reaction is even faster with $\mathrm{CF}_{3}$-DSI (1f), most probably due to steric reasons. Catalyst $\mathbf{1 f}$ is endowed with smaller 3,3'-substituents than both 1 a and $1 \mathbf{e}$, which probably is the decisive factor with bulky substrates such as 2c. 


\subsubsection{1-(4-methylphenyl)- $N$-phenylethan-1-amine $\left(98 \%{ }^{15} \mathrm{~N}\right) 4 \mathrm{~d}$}<smiles>C/C(=N\c1ccccc1)c1ccc(C)cc1</smiles>

2d $0.327 \mathrm{mmol}$<smiles>CCOC(=O)C1=C(C)NC(C)=C(C(=O)OCC)C1</smiles>

$1.4 \mathrm{eq}$

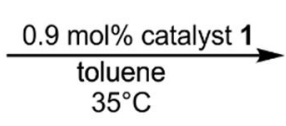

$35^{\circ} \mathrm{C}$<smiles>Cc1ccc(C(C)Nc2ccccc2)cc1</smiles><smiles>CCOC(=O)c1cc(C(=O)OCC)c(C)nc1C</smiles>

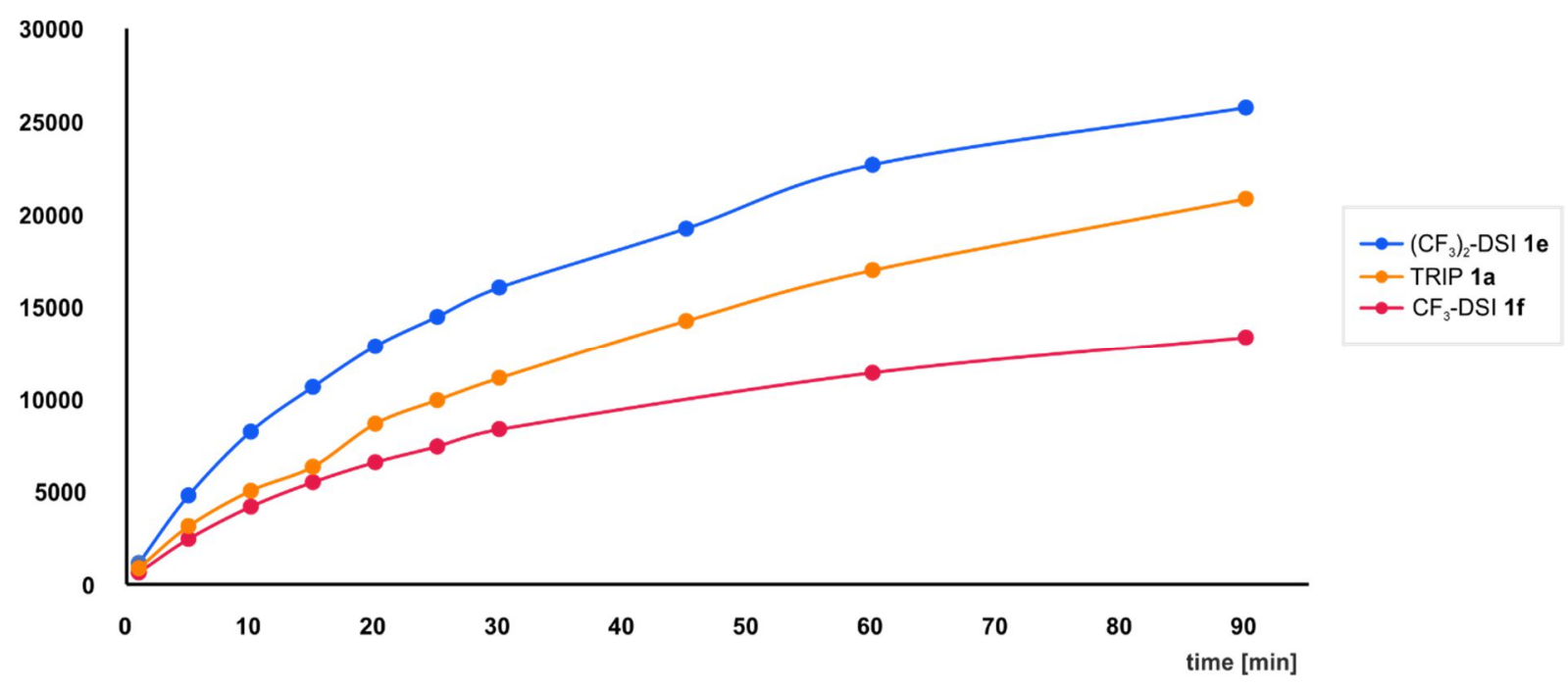

Figure S 45: The formation of $\mathbf{4 d}$ in the asymmetric transfer hydrogenation of imine $\mathbf{2} \mathbf{d}$ is shown. The kinetics were done ex-situ and the samples analyzed by CSP-HPLC. The reaction was conducted with 1.4 equivalents of Hantzsch ester and 0.9 mol\% catalyst with 0.5 eq. $1,3,5$-trimethoxybenzene as the standard at $35^{\circ} \mathrm{C}$ in $\mathrm{PhMe}$.

In the transfer hydrogenation of $\mathbf{2} \mathbf{d}$, the reactivity order with three different catalysts corresponds to the order observed in the transfer hydrogenation of $\mathbf{2 a}$. 


\section{Investigation of $\mathbf{N}$-Tosyl Imine Complexes}

\subsection{Complexes of Imine 9 with TRIP $1 \mathrm{a}$ and DSI 1e}

For a comparison with $\mathrm{N}$-aryl and -alkyl imines, we investigated complexes of $(E)-4$-methyl$\mathrm{N}$-(4-methylbenzylidene)benzenesulfonamide (imine 9), with TRIP 1a and DSI $\left(\mathrm{CF}_{3}\right)_{2}$-DSI 1e by NMR at $180 \mathrm{~K}$. Aldimines populate predominantly the E-configuration, which simplifies the analysis.

Interestingly, the complex of $\mathbf{9}$ with TRIP 1 a does not show a detectable contribution from the structure $1 \mathrm{a} / 9 E_{N}$ with a $\mathrm{POHN}$ hydrogen bond. All of the typical spectroscopic parameter detectable in the case of $\mathrm{POHN}$ hydrogen bonds to $\mathrm{N}$-arylimines even at natural abundance haven't been detected for $\mathrm{N}$-tosylimines. In the corresponding ${ }^{1} \mathrm{H},{ }^{15} \mathrm{~N}$ - or ${ }^{1} \mathrm{H},{ }^{31} \mathrm{P}-\mathrm{HMBC}$ spectra, no correlations that would confirm this structure were found. Additionally, the imine $\mathrm{CH}$ hydrogen is not split by ${ }^{3} \mathrm{~J}_{\mathrm{HH}}$ from the $\mathrm{NH}$ group and does not show any correlation in a 1D selective COSY spectrum (optimized for $8 \mathrm{~Hz}$ couplings).

The basicity of the nitrogen is clearly reduced by the electron-withdrawing sulfonyl group. This is corroborated by the position on the Steiner-Limbach curve (on the very right bottom below the phenols; $\delta{ }^{15} \mathrm{~N}_{\text {ref: }}-33 \mathrm{ppm}$ ), which reveals that only very weak hydrogen bonds to Nitrogen are present if at all. Still multiple $\mathrm{H}$-bonds are present based on the ${ }^{1} \mathrm{H} N M R$ spectrum (major peaks at $\delta 12.50,11.50$ and $6.37 \mathrm{ppm}$, more upfield than $\mathrm{H}$-bonds with $\mathrm{N}$ aryl or -alkyl imines), which are all in mutual slow exchange (EXSY, 150 ms mixing time). This suggests that in complexes with these $N$-tosylimines not the nitrogen, but the oxygens act as hydrogen bond acceptor sites and that a structure such as $1 \mathbf{a} / \mathbf{9 E} E_{o}$ is more likely to be involved. Upon irradiation of the methyl groups, 1D selective NOESY spectrum $(150 \mathrm{~ms}$ mixing time) shows crosspeaks to the whole catalyst backbone, indicating a flexible complex structure resulting from the enlarged structural space of the complex.

In the proton NMR spectrum of the complex of 9 with $\left(\mathrm{CF}_{3}\right)_{2}$-DSI 1e, sharp catalyst/imine peaks but an extremely broad hydrogen bond signal at $\delta 8.80 \mathrm{ppm}$ were observed, precluding any structural analysis. This outcome hints at an ion-pair structure with fast exchange of the hydrogen between multiple acceptor sites. Thus, this result is in agreement with our observation that multiple hydrogen bond acceptor sites (either on the catalyst or substrate) enlarge the structural space in catalysis. However, this does not necessarily lead to lower selectivity, because such catalysts and substrates are routinely used in asymmetric catalysis. ${ }^{[9,10]}$

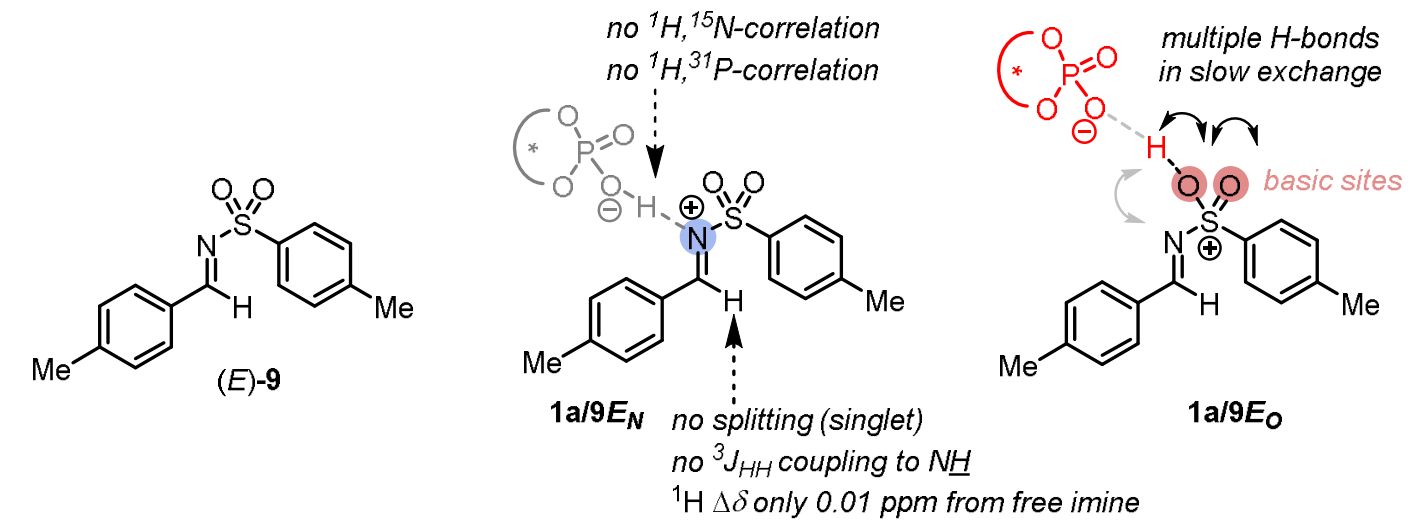

Figure S 46: The structure of imine $\mathbf{9}$, and possible structures for the binary complex of imine 9 and TRIP 1a. 


\section{$11.2{ }^{1} \mathrm{H}$ NMR Spectra of TRIP $1 \mathrm{a} / 9$}

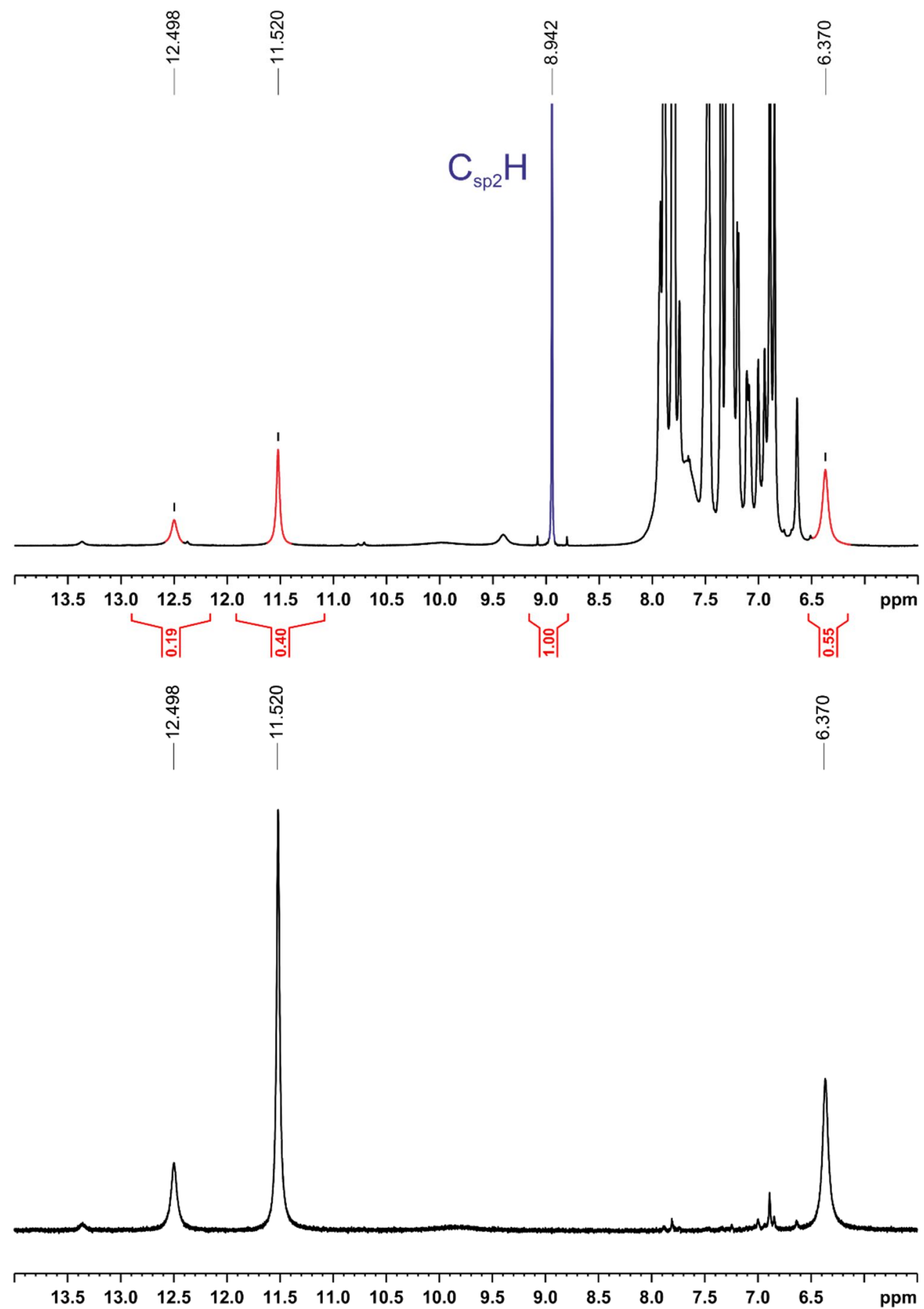

Figure S 47: ${ }^{1} \mathrm{H}$ NMR (top) and ${ }^{1} \mathrm{H} 1 \mathrm{D}$ selective NOESY (bottom) spectra (BF1 = 600.0 MHz; selnogp; NS = 128; DS = 2; $\mathrm{TD}=65536 ; \mathrm{D} 1=2 ; \mathrm{SW}=21.55 \mathrm{ppm} ; \mathrm{O} 1 \mathrm{P}=8.76$, mixing time $\mathrm{D} 8=150 \mathrm{~ms}$ ) of the TRIP $1 \mathrm{a} / 9$ (non- ${ }^{15} \mathrm{~N}$-labelled)complex in $\mathrm{CD}_{2} \mathrm{Cl}_{2}$ at $180 \mathrm{~K}$ irradiated at $\delta 11.52 \mathrm{ppm}$. 
12 Copy of ${ }^{1} \mathrm{H}$ and ${ }^{19} \mathrm{~F}$ NMR Spectra of Catalyst Intermediates

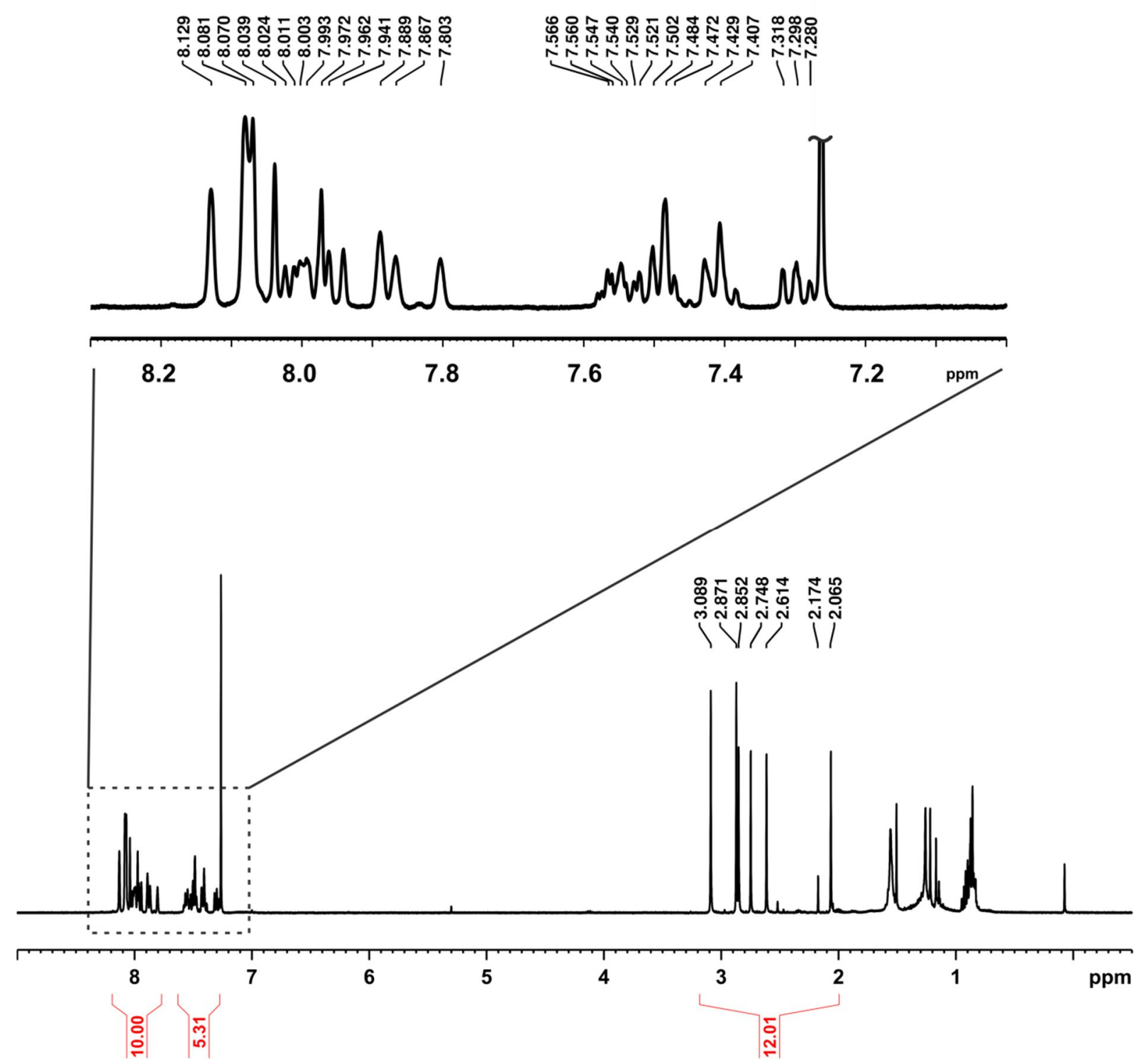

Figure S 48: ${ }^{1} \mathrm{H} \mathrm{NMR}\left(400 \mathrm{MHz}, \mathrm{CDCl}_{3}\right)$ spectrum of 6. 


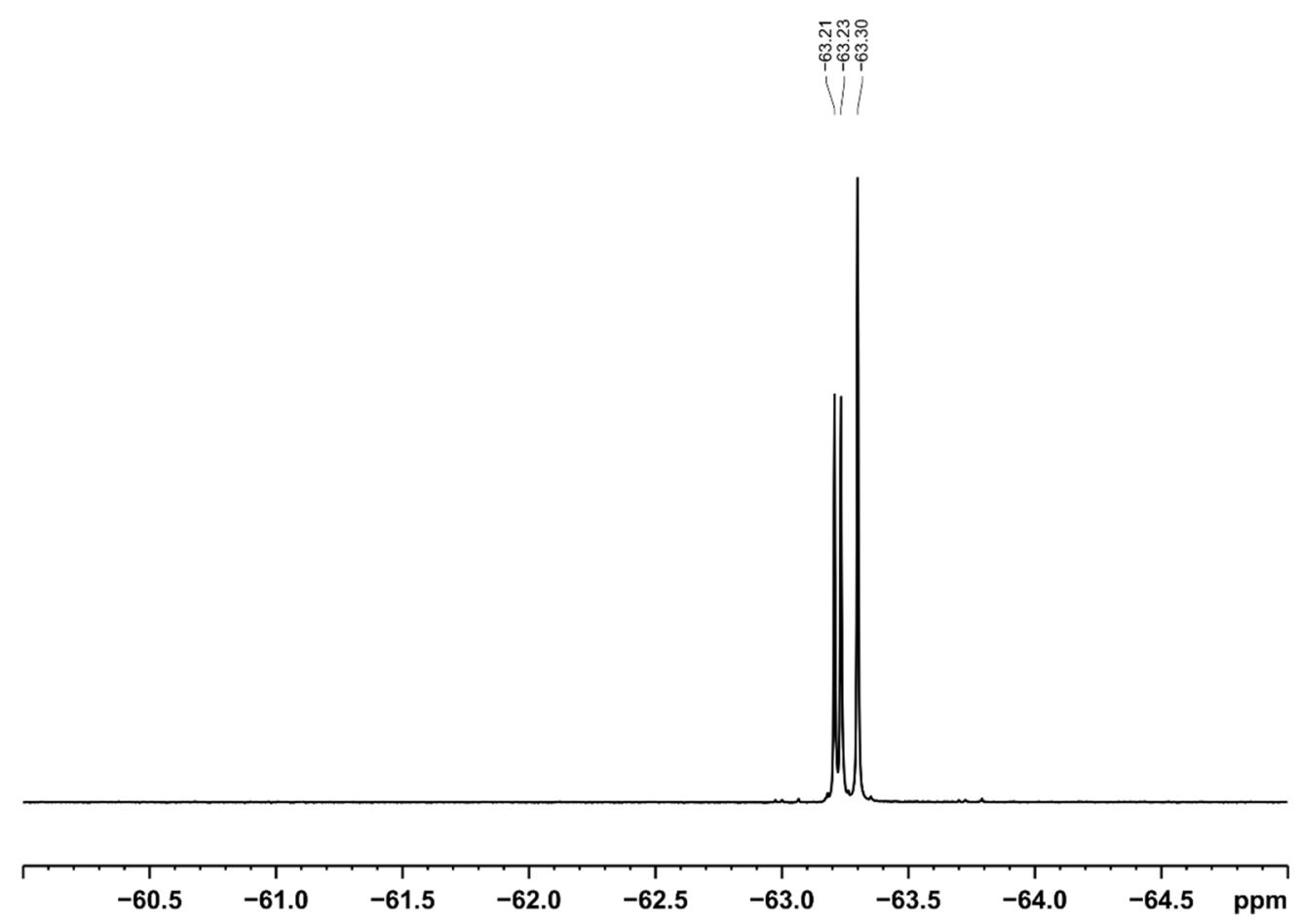

Figure S 49: Portion of ${ }^{19} \mathrm{~F}$ NMR $\left(376 \mathrm{MHz}, \mathrm{CDCl}_{3}\right)$ spectrum of 6. 


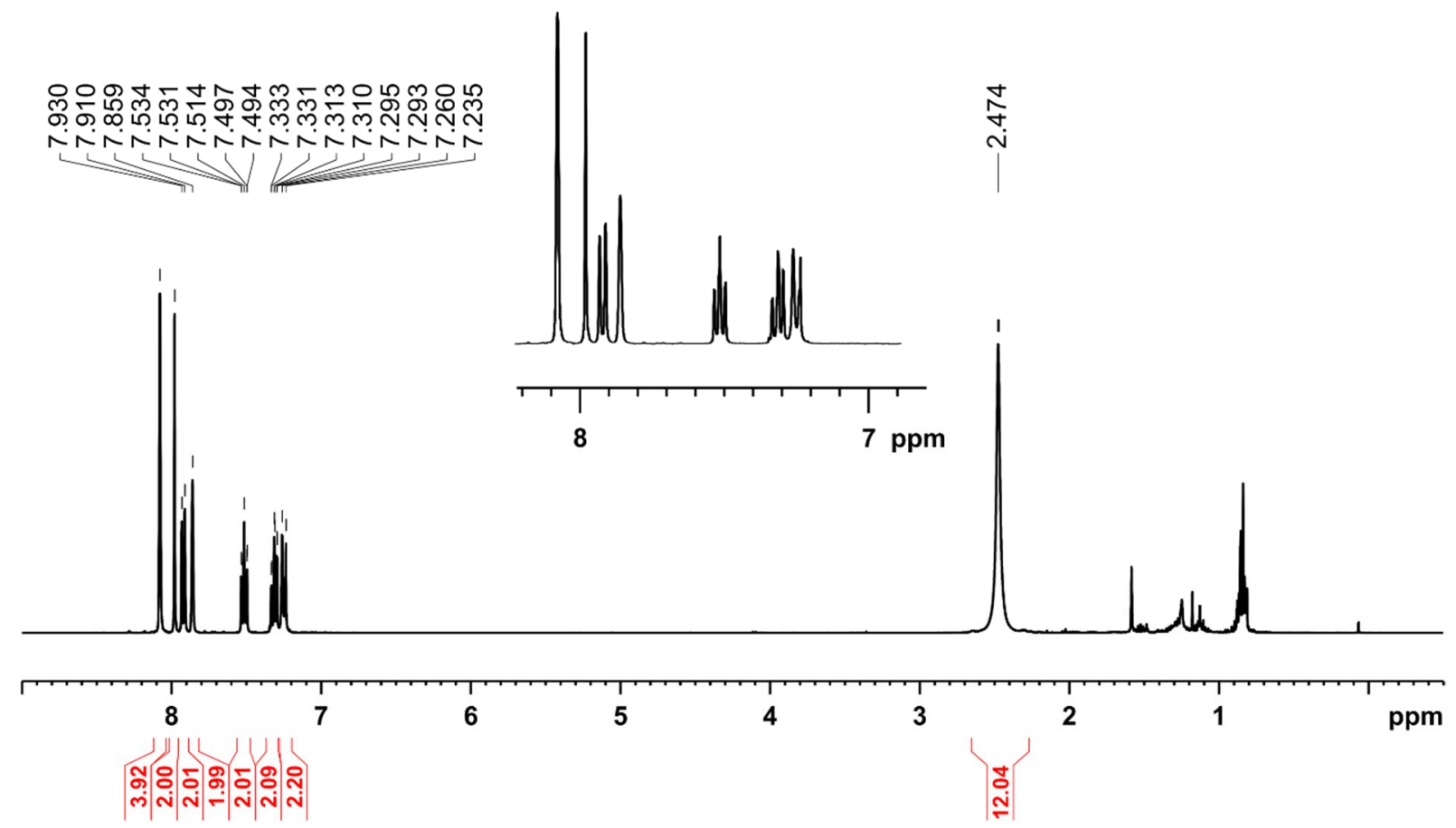

Figure S 50: ${ }^{1} \mathrm{H}$ NMR $\left(400 \mathrm{MHz}, \mathrm{CDCl}_{3}\right)$ spectrum of 7.

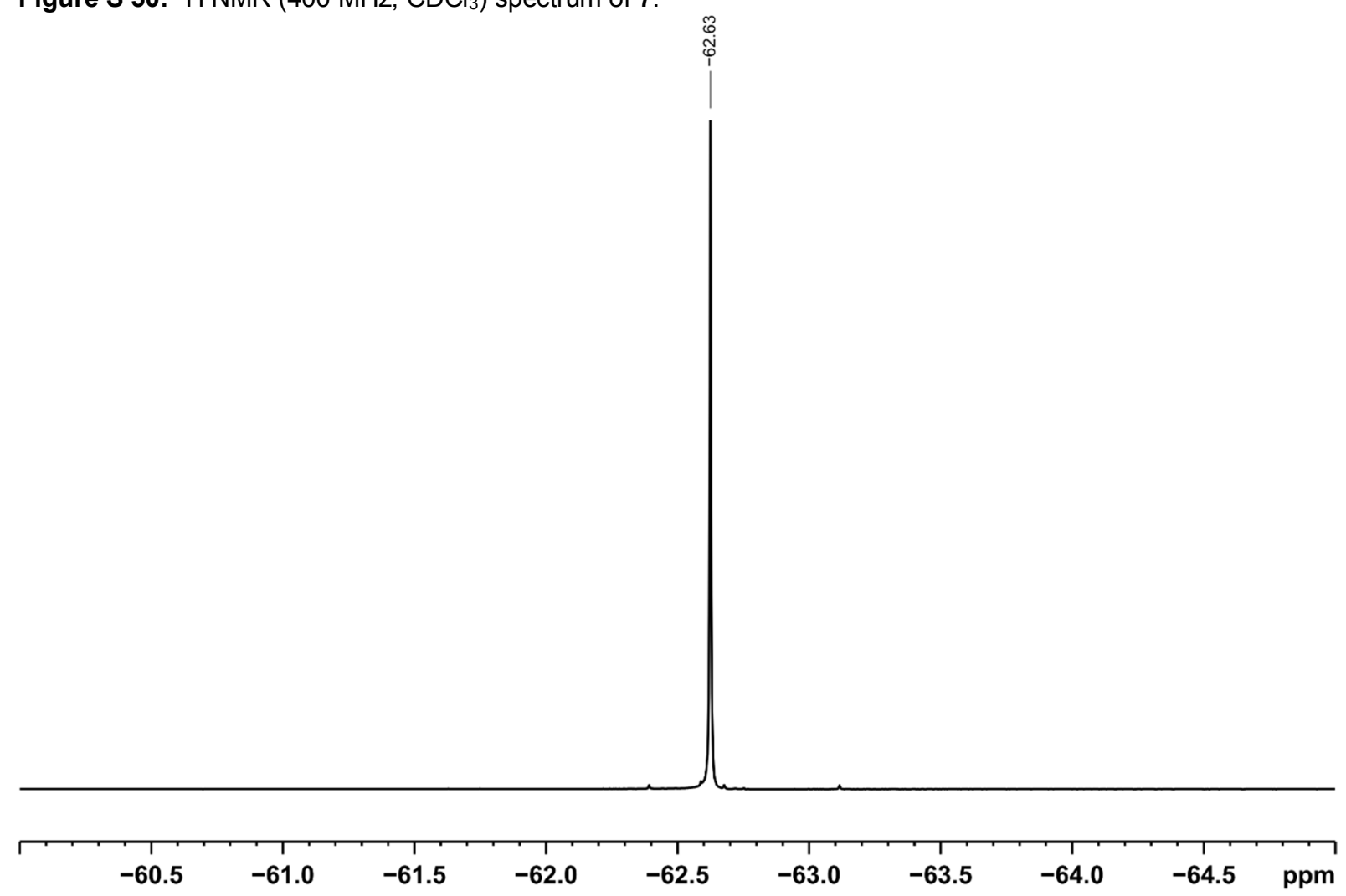

Figure S 51: Portion of $\left.{ }^{19} \mathrm{~F} \mathrm{NMR} \mathrm{(376} \mathrm{MHz,} \mathrm{CDCl}_{3}\right)$ spectrum of 7 . 


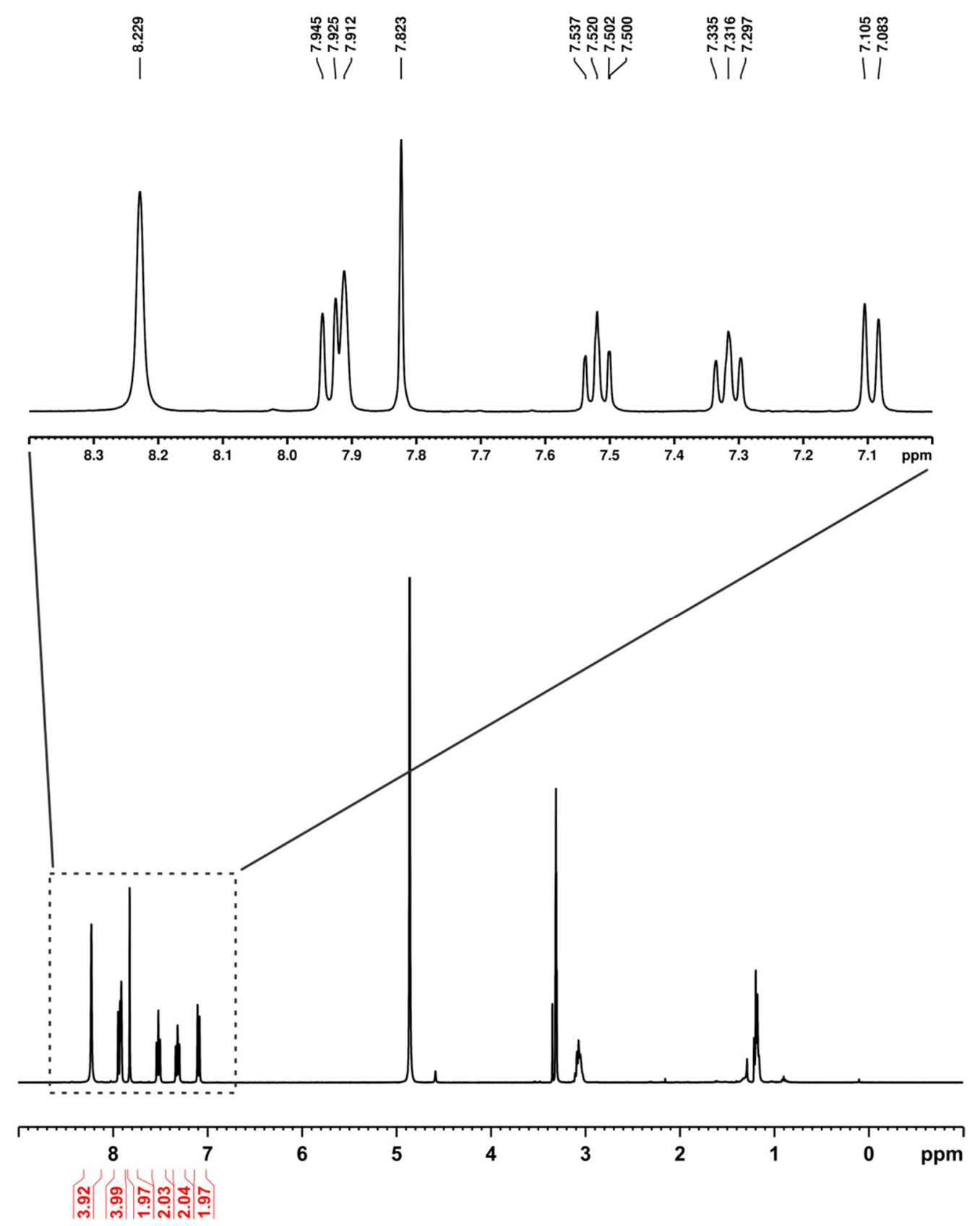

Figure S 52: ${ }^{1} \mathrm{H}$ NMR $\left(400 \mathrm{MHz}, \mathrm{CD}_{3} \mathrm{OD}\right)$ spectrum of $\mathbf{1 g}$. 


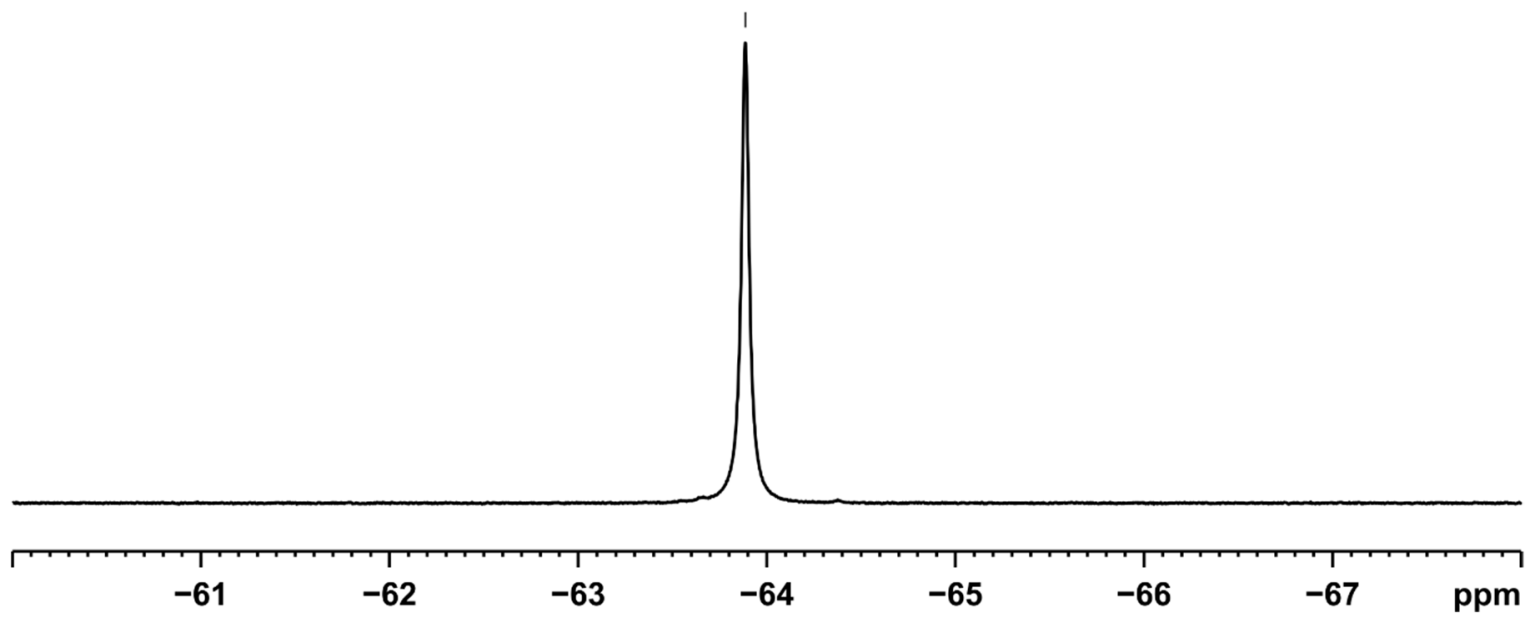

Figure S 53: Portion of ${ }^{19} \mathrm{~F}$ NMR $\left(376 \mathrm{MHz}, \mathrm{CD}_{3} \mathrm{OD}\right)$ spectrum of $\mathbf{1 g}$. 


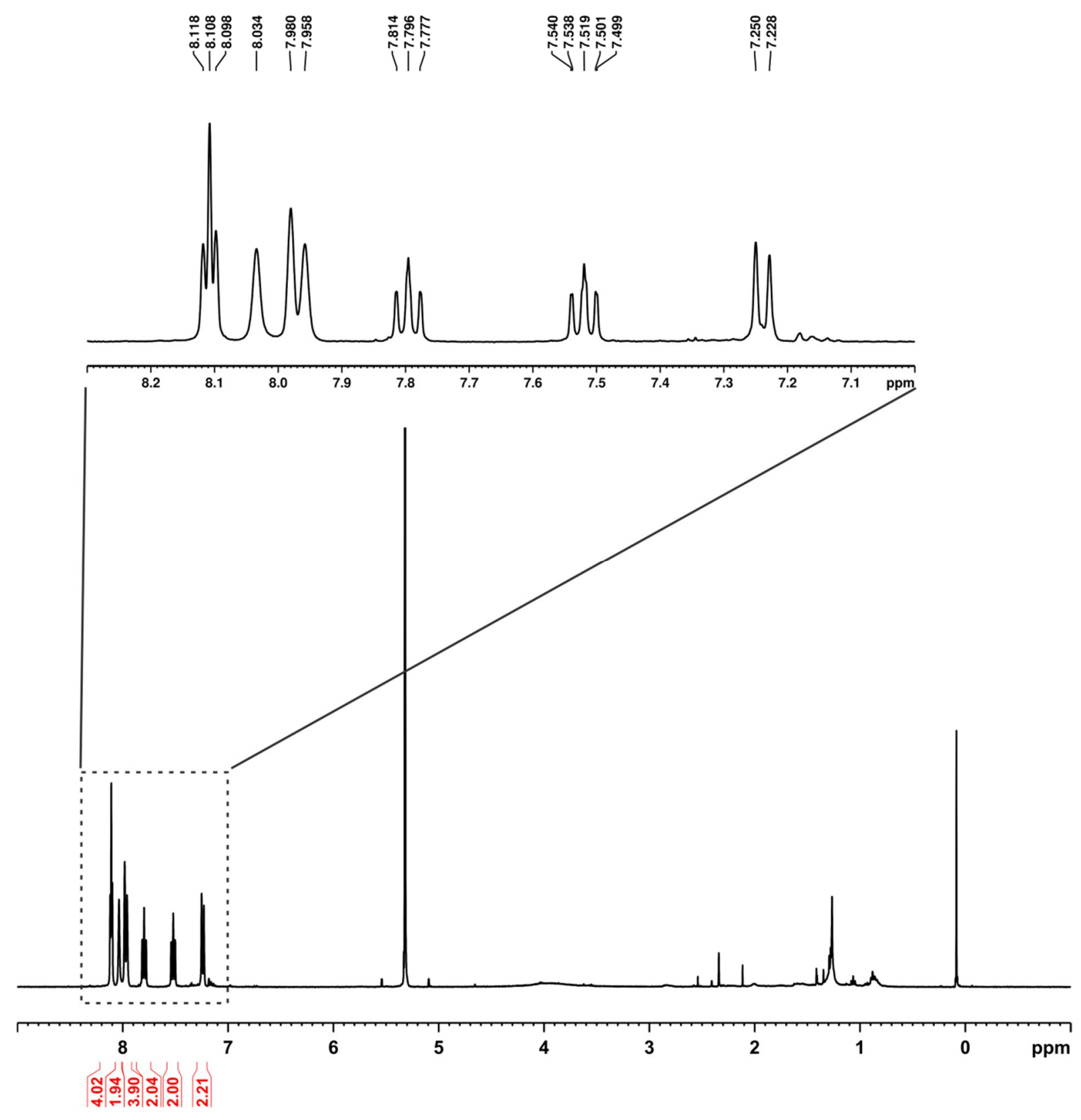

Figure S 54: ${ }^{1} \mathrm{H}$ NMR $\left(400 \mathrm{MHz}, \mathrm{CD}_{2} \mathrm{Cl}_{2}\right)$ spectrum of ${ }^{15} \mathrm{~N}$-labelled $1 \mathrm{e}$. 
ஜํㅇㅇ

ชิ่ ญิ

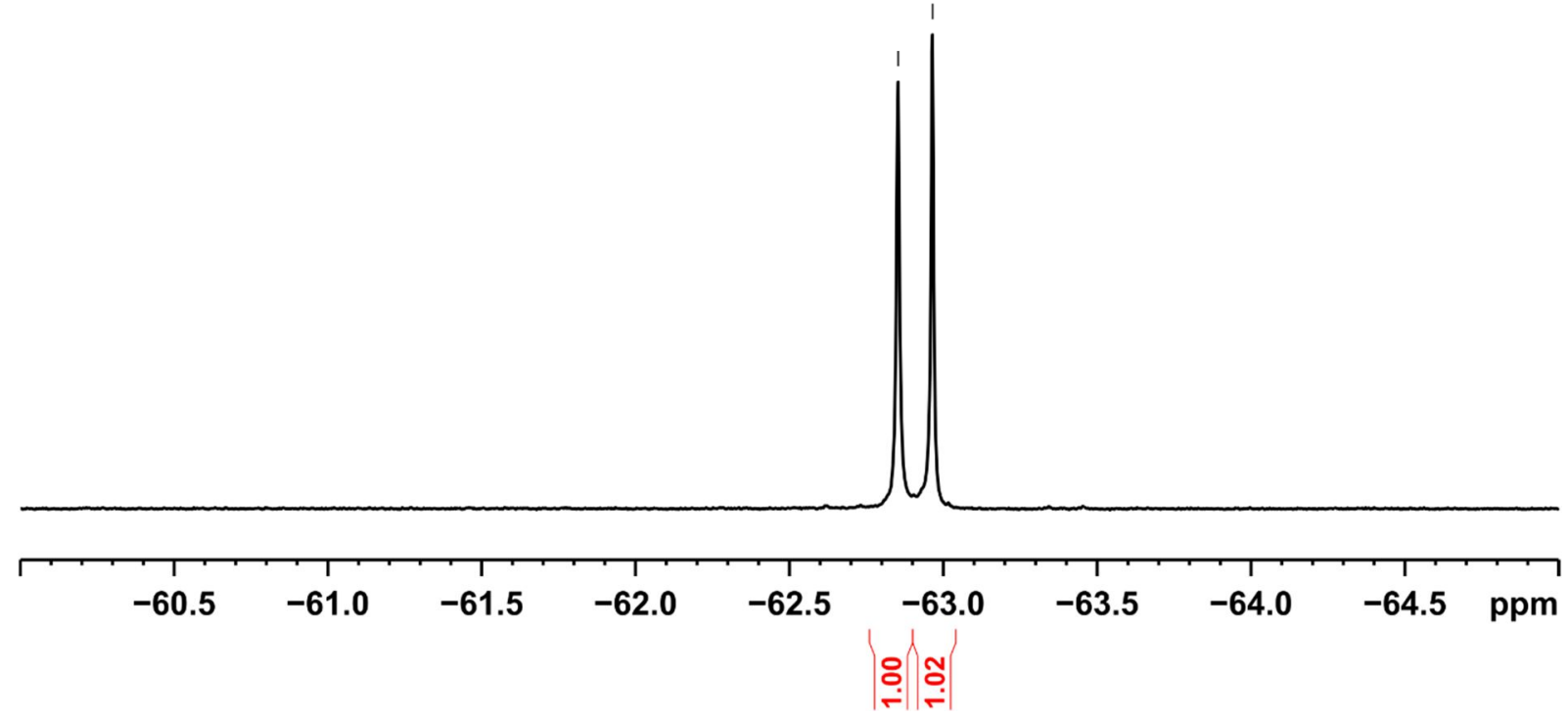

Figure S 55: Portion of ${ }^{19} \mathrm{~F} \mathrm{NMR}\left(376 \mathrm{MHz}, \mathrm{CD}_{2} \mathrm{Cl}_{2}\right)$ spectrum of ${ }^{15} \mathrm{~N}$-labelled $1 \mathrm{e}$. 


\section{References}

[1] N. Sorgenfrei, J. Hioe, J. Greindl, K. Rothermel, F. Morana, N. Lokesh, R. M. Gschwind, J. Am. Chem. Soc. 2016, 138, 16345-16354.

[2] K. Rothermel, M. Melikian, J. Hioe, J. Greindl, J. Gramüller, M. Zabka, N. Sorgenfrei, T. Hausler, F. Morana, R. M. Gschwind, Chem. Sci. 2019, accepted. https://doi.org/10.1039/C9SC02342A.

[3] S. Sharif, G. S. Denisov, M. D. Toney, H. Limbach, J. Am. Chem. Soc. 2007, 129, 6313-6327.

[4] P. García-García, F. Lay, P. García-García, C. Rabalakos, B. List, Angew. Chemie Int. Ed. 2009, 48, 4363-4366.

[5] Gaussian 09, Revision D.01, M. J. Frisch, G. W. Trucks, H. B. Schlegel, G. E. Scuseria, M. A. Robb, J. R. Cheeseman, G. Scalmani, V. Barone, B. Mennucci, G. A. Petersson, H. Nakatsuji, M. Caricato, X. Li, H. P. Hratchian, A. F. Izmaylov, J. Bloino, G. Zheng, J. L. Sonnenberg, M. Hada, M. Ehara, K. Toyota, R. Fukuda, J. Hasegawa, M. Ishida, T. Nakajima, Y. Honda, O. Kitao, H. Nakai, T. Vreven, J. A. Montgomery, Jr., J. E. Peralta, F. Ogliaro, M. Bearpark, J. J. Heyd, E. Brothers, K. N. Kudin, V. N. Staroverov, R. Kobayashi, J. Normand, K. Raghavachari, A. Rendell, J. C. Burant, S. S. lyengar, J. Tomasi, M. Cossi, N. Rega, J. M. Millam, M. Klene, J. E. Knox, J. B. Cross, V. Bakken, C. Adamo, J. Jaramillo, R. Gomperts, R. E. Stratmann, O. Yazyev, A. J. Austin, R. Cammi, C. Pomelli, J. W. Ochterski, R. L. Martin, K. Morokuma, V. G. Zakrzewski, G. A. Voth, P. Salvador, J. J. Dannenberg, S. Dapprich, A. D. Daniels, Ö. Farkas, J. B. Foresman, J. V. Ortiz, J. Cioslowski, and D. J. Fox, Gaussian, Inc., Wallingford CT, 2009.

[6] P. Renzi, J. Hioe, R. M. Gschwind, J. Am. Chem. Soc. 2017, 139, 6752-6760.

[7] C. Yang, X. S. Xue, X. Li, J. P. Cheng, J. Org. Chem. 2014, 79, 4340-4351.

[8] J. P. Reid, J. M. Goodman, J. Am. Chem. Soc. 2016, 138, 7910-7917.

[9] Q. Kang, Z. A. Zhao, S. L. You, J. Am. Chem. Soc. 2007, 129, 1484-1485.

[10] E. P. Ávila, R. M. S. Justo, V. P. Gonçalves, A. A. Pereira, R. Diniz, G. W. Amarante, J. Org. Chem. 2015, 80, 590-594. 